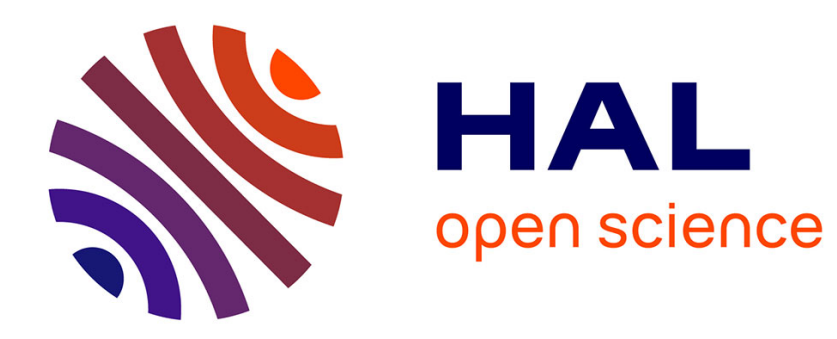

\title{
Inverse scattering at fixed energy on three-dimensional asymptotically hyperbolic Stäckel manifolds
}

\author{
Damien Gobin
}

\section{To cite this version:}

Damien Gobin. Inverse scattering at fixed energy on three-dimensional asymptotically hyperbolic Stäckel manifolds. 2016. hal-01316184

\author{
HAL Id: hal-01316184 \\ https://hal.science/hal-01316184 \\ Preprint submitted on 15 May 2016
}

HAL is a multi-disciplinary open access archive for the deposit and dissemination of scientific research documents, whether they are published or not. The documents may come from teaching and research institutions in France or abroad, or from public or private research centers.
L'archive ouverte pluridisciplinaire HAL, est destinée au dépôt et à la diffusion de documents scientifiques de niveau recherche, publiés ou non, émanant des établissements d'enseignement et de recherche français ou étrangers, des laboratoires publics ou privés. 


\title{
Inverse scattering at fixed energy on three-dimensional asymptotically hyperbolic Stäckel manifolds
}

\author{
Damien Gobin*
}

May 15, 2016

\begin{abstract}
In this paper, we study an inverse scattering problem at fixed energy on three-dimensional asymptotically hyperbolic Stäckel manifolds having the topology of toric cylinders and satisfying the Robertson condition. On these manifolds the Helmholtz equation can be separated into a system of a radial ODE and two angular ODEs. We can thus decompose the full scattering operator onto generalized harmonics and the resulting partial scattering matrices consist in a countable set of $2 \times 2$ matrices whose coefficients are the so-called transmission and reflection coefficients. It is shown that the reflection coefficients are nothing but generalized Weyl-Titchmarsh functions associated with the radial ODE. Using a novel multivariable version of the Complex Angular Momentum method, we show that the knowledge of the scattering operator at a fixed non-zero energy is enough to determine uniquely the metric of the three-dimensional Stäckel manifold up to natural obstructions.
\end{abstract}

Keywords. Inverse Scattering, Stäckel manifolds, CAM method, Weyl-Titchmarsh function. 2010 Mathematics Subject Classification. Primaries 81U40, 35P25; Secondary 58J50.

\section{Introduction and statement of the main result}

In this work we are interested in an inverse scattering problem at fixed energy for the Helmholtz equation on three-dimensional Stäckel manifolds satisfying the Robertson condition. The Stäckel manifolds were introduced in 1891 by Stäckel in [68] and are of main interest in the theory of variable separation. Indeed, it is known that the additive separability of the Hamilton-Jacobi equation for the geodesic flow on a riemannian manifold is equivalent to the fact that the metric is in Stäckel form. However, to obtain the multiplicative separability of the Helmholtz equation, we also have to assume that the Robertson condition is satisfied. As we will see in a brief review of the theory of variable separation, there also exist some intrinsic characterizations of the separability of the Hamilton-Jacobi and Helmholtz equations in terms of Killing tensors (which correspond to hidden symmetries) or symmetry operators. We refer to [5, 6, 17, 29, 30, 43, 44, 45, 69, for important contributions in this domain and to [3, 59] for surveys on these questions. We emphasize that the description of the riemannian manifolds given by Stäckel is local. We shall obtain a global description of these manifolds by choosing a global Stäckel system of coordinates and we shall thus use the name of "Stäckel manifold" in our study. We choose to work on a Stäckel manifold $(\mathcal{M}, g)$ having the topology of a toric cylinder and in order to define the scattering matrix on

${ }^{*}$ Laboratoire de Mathématiques Jean Leray, Université de Nantes, 2, rue de la Houssinière, BP 92208, 44322 Nantes Cedex 03. Email adress: damien.gobin@univ-nantes.fr. Research supported by the French National Research Project AARG, No. ANR-12-BS01-012-01. 
this manifold, we add an asymptotically hyperbolic structure, introduced in [40] (see also [35, 42, 65), at the two radial ends of our cylinder. We can then define the scattering operator $S_{g}(\lambda)$ at a fixed energy $\lambda \neq 0$ associated with the Helmholtz equation on $(\mathcal{M}, g)$ which is the object of main interest of this paper. The question we address is the following:

\section{Does the scattering operator $S_{g}(\lambda)$ at a fixed energy $\lambda \neq 0$ uniquely determine the metric $g$ of the Stäckel manifold?}

We recall that inverse scattering problems at fixed energy on asymptotically hyperbolic manifolds are closely related to the anisotropic Calderón problem on compact riemannian manifolds with boundary. We refer to the surveys 38, 40, 46, 48, 66, 71, for the current state of the art on this question. One of the aim of this paper is thus to give examples of manifolds on which we can solve the inverse scattering problem at fixed energy but do not have one of the particular structures for which the uniqueness for the anisotropic Calderón problem on compact manifolds with boundary is known. Note that the result we prove here is an uniqueness result. We are also interested in stability result i.e. in study the continuity of the application $g \mapsto S_{g}(\lambda)$. This question will be the object of a future work.

The main tool of this paper consists in complexifying the coupled angular momenta that appear in the variable separation procedure. Indeed, thanks to variable separation, the scattering operator at fixed energy can be decomposed onto scattering coefficients indexed by a discrete set of two angular momenta that correspond to the two constants of separation. Roughly speaking, the aim of the Complexification of the Angular Momentum method is the following: from a discrete set of data (here the equality of the reflection coefficients on the coupled spectrum) we want to obtain a continuous regime of informations (here the equality of these functions on $\mathbb{C}^{2}$ ). This method consists in three steps. We first allow the angular momentum to be a complex number. We then use uniqueness results for functions in certain analytic classes to obtain the equality of the non-physical corresponding data on the complex plane $\mathbb{C}$. Finally, we use this new information to solve our inverse problem thanks to the Borg-Marchenko Theorem. The general idea of considering complex angular momentum originates from a paper of Regge (see [63) as a tool in the analysis of the scattering matrix of Schrödinger operators in $\mathbb{R}^{3}$ with spherically symmetric potentials. We also refer to [1, 61 for books dealing with this method. This tool was already used in the field of inverse problems for one angular momentum in $[21,22,23,24,25,26,34,62$ and we note that this method is also used in high energy physics (see 20]). In this work we use a novel multivariable version of the Complexification of the Angular Momentum method for two angular momenta which correspond to the constants of separation of the Helmholtz equation. Note that we have to use this multivariable version since these two angular moments (which are also coupled eigenvalues of two commuting operators) are not independent and cannot be considered separately. This work is a continuation of the paper 22 of Daudé, Kamran and Nicoleau in which the authors treat the same question on Liouville surfaces which correspond to Stäckel manifolds in two dimensions.

\subsection{Review of variable's separation theory}

In this Subsection, we propose a brief review of variable's separation theory. We refer to [3, 59] and to the introduction of $[5$ for surveys of this theory. Let $(\mathcal{M}, g)$ be a riemannian manifold of dimension $n$. On $(\mathcal{M}, g)$, we are first interested in the Hamilton-Jacobi equation

$$
\nabla W . \nabla W=E,
$$

where $E$ is a constant parameter and $\nabla$ is the gradient operator

$$
(\nabla W)^{i}=g^{i j} \partial_{j} W,
$$


where we use the Einstein summation convention. We are also interested in the Helmholtz equation

$$
-\Delta_{g} \psi=E \psi .
$$

where $\Delta_{g}$ is the Laplace-Beltrami operator

$$
\Delta_{g} \psi=g^{i j} \nabla_{i} \nabla_{j} \psi,
$$

where $\nabla_{j}$ is the covariant derivative with respect to the Levi-Civita connection. We note that, as it is shown in [5, we can add a potential $V$ satisfying suitable conditions to these equations without more complications in the theory we describe here. It is known that, in many interesting cases, these equations admit local separated solutions of the form

$$
W(\underline{x}, \underline{c})=\sum_{i=1}^{n} W_{i}\left(x^{i}, \underline{c}\right),
$$

for the Hamilton-Jacobi equation and of the form

$$
\psi(\underline{x}, \underline{c})=\prod_{i=1}^{n} \psi_{i}\left(x^{i}, \underline{c}\right)
$$

for the Helmholtz equation, where $\underline{x}=\left\{x^{i}\right\}$ is a suitable coordinate system on $\mathcal{M}$ and $\underline{c}$ denotes a set of constant parameters, whose number depends on an appropriate definition of separation (see below). The reason why we are interested in such solutions is that it happens that for solutions of this kind, Equations 1.1)-(1.2) become equivalent to a system of ordinary differential separated equations, each one involving a single coordinate. In this work we study the particular case of the orthogonal separation, i.e. when $g^{i j}=0$ for $i \neq j$. We now recall the definition of separation of variables for the Hamilton-Jacobi and the Helmholtz equations.

Definition 1.1 ([5]). The Hamilton-Jacobi equation is separable in the coordinates $\underline{x}=\left\{x^{i}\right\}$ if it admits a complete separated solution, i.e. a solution of the kind

$$
W(\underline{x}, \underline{c})=\sum_{i=1}^{n} W_{i}\left(x^{i}, \underline{c}\right),
$$

depending on $n$ parameters $\underline{c}=\left(c_{j}\right)$ satisfying the completeness condition

$$
\operatorname{det}\left(\frac{\partial p_{i}}{\partial c_{j}}\right) \neq 0, \text { where } p_{i}=\partial_{i} W .
$$

Definition 1.2 ([ $\left[\underline{5}\right.$, Definition 4.1). The Helmholtz equation is separable in the coordinates $\underline{x}=\left\{x^{i}\right\}$ if it admits a complete separated solution, i.e. a solution of the form

$$
\psi(\underline{x}, \underline{c})=\prod_{i=1}^{n} \psi_{i}\left(x^{i}, \underline{c}\right),
$$

depending on $2 n$ parameters $\underline{c}=\left(c_{j}\right)$ satisfying the completeness condition

$$
\operatorname{det}\left(\begin{array}{c}
\frac{\partial u_{i}}{\partial c_{j}} \\
\frac{\partial v_{i}}{\partial c_{j}}
\end{array}\right) \neq 0, \quad \text { where } \quad u_{i}=\frac{\psi_{i}^{\prime}}{\psi_{i}} \quad \text { and } \quad v_{i}=\frac{\psi_{i}^{\prime \prime}}{\psi_{i}} .
$$


We now recall the results proved by Stäckel, Robertson and Eisenhart at the end of the eighteenth century and at the beginning of the nineteenth century which:

1. Characterize the riemannian manifolds admitting orthogonal variable separation.

2. Make the link between the variable separation for Hamilton-Jacobi and Helmholtz equations.

Definition 1.3 (Stäckel matrix). A Stäckel matrix is a regular $n \times n$ matrix $S(\underline{x})=\left(s_{i j}\left(x^{i}\right)\right)$ whose components $s_{i j}\left(x^{i}\right)$ are functions depending on the variable corresponding to the line number only.

Theorem 1.1 (Stäckel 1893, 69]). The Hamilton-Jacobi equation is separable in orthogonal coordinates $\underline{x}$ if and only if the metric $g$ is of the form

$$
g=H_{1}^{2}\left(d x^{1}\right)^{2}+H_{2}^{2}\left(d x^{2}\right)^{2}+H_{3}^{2}\left(d x^{3}\right)^{2},
$$

where

$$
H_{i}^{2}=\frac{\operatorname{det}(S)}{s^{i 1}}, \quad \forall i \in\{1,2,3\},
$$

where $s^{i 1}$ is the minor associated with the coefficient $s_{i 1}$, for all $i \in\{1,2,3\}$.

Theorem 1.2 (Robertson 1927, 64]). The Helmholtz equation is separable in orthogonal coordinates $\underline{x}$ if and only if in these coordinates the Hamilton-Jacobi equation is separable and moreover the following condition is satisfied

$$
\frac{\operatorname{det}(S)^{2}}{|g|}=\frac{\operatorname{det}(S)^{2}}{\prod_{i=1}^{n} H_{i}^{2}}=\prod_{i=1}^{n} f_{i}\left(x^{i}\right)
$$

where $f_{i}\left(x^{i}\right)$ are arbitrary functions of the corresponding coordinate only and $|g|$ is the determinant of the metric $g$.

Thanks to this Theorem we see that a full understanding of the separation theory for the Helmholtz equation depends on an understanding of the corresponding problem for the Hamilton-Jacobi equation and we note that the separability of the Helmholtz equation is more demanding. The additional condition 1.3 in Theorem 1.2 is called Robertson condition. This condition has a geometrical meaning given by the following characterization dues to Eisenhart.

Theorem 1.3 (Eisenhart 1934, 29]). The Robertson condition (1.3) is satisfied if and only if in the orthogonal coordinates system $\underline{x}$ the Ricci tensor is diagonal:

$$
R_{i j}=0, \quad \forall i \neq j .
$$

Remark 1.4. We note that the Robertson condition is satisfied for Einstein manifolds. Indeed, an Einstein manifold is a riemannian manifold whose Ricci tensor is proportional to the metric which is diagonal in the orthogonal case we study.

As shown by Eisenhart in [29, 30] and by Kalnins and Miller in 44, the separation of the HamiltonJacobi equation for the geodesic flow is related to the existence of Killing tensors of order two (whose presence highlights the presence of hidden symmetries). We thus follow [3, 44] in order to study this relation. We use the natural symplectic structure on the cotangent bundle $T^{\star} \mathcal{M}$ of the manifold $(\mathcal{M}, g)$. Let $\left\{x^{i}\right\}$ be local coordinates on $\mathcal{M}$ and $\left\{x^{i}, p_{i}\right\}$ the associated coordinates on $T^{\star} \mathcal{M}$. Let

$$
H=g^{i j} p_{i} p_{j},
$$


be the geodesic hamiltonian on $T^{\star} \mathcal{M}$. The Hamilton-Jacobi equation can thus be written as

$$
H\left(x^{i}, \partial_{i} W\right)=E .
$$

Thanks to this formalism, an other necessary and sufficient condition for the separability of the HamiltonJacobi equation has been proved by Levi-Civita. We state the version given in [3].

Theorem 1.5 (Levi-Civita 1904, [55]). The Hamilton-Jacobi equation

$$
H\left(x^{i}, \partial_{i} W\right)=E,
$$

admits a separated solution if and only if the differential equations, known as the separability equations of Levi-Civita,

$$
L_{i j}(H):=\partial_{i} H \partial_{j} H \partial^{i} \partial^{j} H+\partial^{i} H \partial^{j} \partial_{i} \partial_{j} H-\partial_{i} H \partial^{j} H \partial^{i} \partial_{j} H-\partial^{i} H \partial_{j} H \partial_{i} \partial^{j} H=0,
$$

where $\partial_{i}=\frac{\partial}{\partial x^{i}}$ and $\partial^{i}=\frac{\partial}{\partial p_{i}}$, are identically satisfied.

Remark 1.6. This Theorem gives us a simple method for testing whether a coordinate system is separable or not. Moreover, it also provides us the basis for the geometrical (i.e. intrinsic) characterization of the separation.

Since we are interested in a characterization of the separation of the Hamilton-Jacobi equation using Killing tensors, we recall here some basic properties of these objects following the second Section of [3]. We first recall that the contravariant symmetric tensors $\mathbf{K}=\left(K^{i \ldots j}\right)$ on $\mathcal{M}$ are in one-to-one correspondence with the homogeneous polynomials on $T^{\star} \mathcal{M}$ by the correspondence,

$$
P_{\mathbf{K}}=P(\mathbf{K})=K^{i \ldots j} p_{i} \ldots p_{j} .
$$

Example 1.1. The hamiltonian $H$ corresponds to the contravariant metric tensor $\mathbf{G}$.

The space of these polynomial functions is closed with respect to the canonical Poisson bracket

$$
\{A, B\}:=\partial^{i} A \partial_{i} B-\partial^{i} B \partial_{i} A .
$$

We then defined the Lie-algebra structure [.,.] on the space of the symmetric contravariant tensors by setting,

$$
P\left(\left[\mathbf{K}_{1}, \mathbf{K}_{2}\right]\right)=\left\{P\left(\mathbf{K}_{1}\right), P\left(\mathbf{K}_{2}\right)\right\} .
$$

Definition 1.4. Let $\mathbf{G}$ be a metric tensor. We say that $\mathbf{K}$ is a Killing tensor if $P(\mathbf{K})$ is in involution with $P_{\mathbf{G}}=P(\mathbf{G})=H$, i.e.

$$
\{P(\mathbf{K}), P(\mathbf{G})\}=0
$$

which, by definition, is equivalent to the Killing equation,

$$
[\mathbf{K}, \mathbf{G}]=0 .
$$

Remark 1.7. 1. This means that if $\mathbf{K}$ is a Killing tensor, $P(\mathbf{K})$ is a first integral of the geodesic flow.

2. A vector field $\mathbf{X}$ is a Killing vector field, i.e. $[\mathbf{X}, \mathbf{G}]=0$, if and only if its flow preserves the metric. 
We now consider the case of a symmetric 2-tensor $\mathbf{K}$. Since there is a metric tensor, $\mathbf{K}$ can be represented in components as a tensor of type $(2,0),(1,1)$ or $(0,2)$, respectively noted $\mathbf{K}=\left(K^{i j}\right)=$ $\left(K_{j}^{i}\right)=\left(K_{i j}\right)$. As a symmetric tensor of type $(1,1)$, $\mathbf{K}$ defines an endomorphism on the space $\chi(\mathcal{M})$ of the vector fields on $\mathcal{M}$ and an endomorphism on the space $\Phi^{1}(\mathcal{M})$ of the one-forms on $\mathcal{M}$. We denote by $\mathbf{K X}$ the vector field image of $\mathbf{X} \in \chi(\mathcal{M})$ by $\mathbf{K}$ and by $\mathbf{K} \phi$ the one-form image of $\phi \in \Phi^{1}(\mathcal{M})$ by $\mathbf{K}$. In other words,

$$
\mathbf{K X}=K_{j}^{i} X^{j} \partial_{i} \quad \text { and } \quad \mathbf{K} \phi=K_{j}^{i} \phi_{i} d x^{j}
$$

Then a 2-tensor $\mathbf{K}$ gives rise to eigenvalues, eigenvectors or eigenforms according to the equations $\mathbf{K X}=$ $\rho \mathbf{X}$ and $\mathbf{K} \phi=\rho \phi$. Finally, we denote by $\mathbf{K}_{1} \mathbf{K}_{2}$ the product of the two endomorphisms $\mathbf{K}_{1}$ and $\mathbf{K}_{2}$ whose expression in components is $\mathbf{K}_{1} \mathbf{K}_{2}=K_{1}^{i h} K_{2 h}^{j}$. The algebraic commutator of two tensors is then denoted by

$$
\llbracket \mathbf{K}_{1}, \mathbf{K}_{2} \rrbracket:=\mathbf{K}_{1} \mathbf{K}_{2}-\mathbf{K}_{2} \mathbf{K}_{1} .
$$

The first link between separation of variables for Hamilton-Jacobi equation and Killing tensors is then given by the following Proposition.

Proposition 1.8 ([7, 44, 49]). To every orthogonal coordinate system $\left\{x^{i}\right\}$ which permits additive separation of variables in the Hamilton-Jacobi equation, there correspond $n-1$ second order Killing tensors $\mathbf{K}_{1}, \ldots, \mathbf{K}_{n-1}$ in involution, i.e. $\left[\mathbf{K}_{i}, \mathbf{K}_{j}\right]=0$, and such that $\left\{H, P_{\mathbf{K}_{1}}, \ldots, P_{\mathbf{K}_{n-1}}\right\}$ is linearly independent. The separable solutions $W=\sum_{k=1}^{n} W^{(k)}\left(x^{k}\right)$ are characterized by the relations

$$
H\left(x^{i}, p_{i}\right)=E \quad \text { and } \quad P_{\mathbf{K}_{l}}\left(x^{i}, p_{i}\right)=\lambda_{l}, \quad \text { where } \quad l=1, \ldots, n-1 \quad \text { and } \quad p_{i}=\partial_{x^{i}} W
$$

where $\lambda_{1}, \ldots, \lambda_{n-1}$ are the separation constants.

Remark 1.9. In language of hamiltonian mechanics, Killing tensors correspond to constants of the motion. The link mentioned in the previous Proposition thus states that if the Hamilton-Jacobi equation is separable, then the corresponding hamiltonian system is completely integrable (see [2]).

Whereas it corresponds a family of $n-1$ Killing tensors in involution to every separable system, there also exist families of Killing tensors in involution that are not related to separable systems. We thus need to find additional conditions to characterize the admissible families of Killing tensors. Such conditions are given in the following two Theorems.

Theorem 1.10 ([5] Theorem 7.15). The Hamilton-Jacobi equation is orthogonally separable if and only if there exist $n$ pointwise independent Killing tensors $\left(\mathbf{K}_{i}\right)$ one other commuting as linear operators, i.e. $\llbracket \mathbf{K}_{i}, \mathbf{K}_{j} \rrbracket=0$, for all $i \neq j$, and in involution, i.e. $\left[\mathbf{K}_{i}, \mathbf{K}_{j}\right]=0$, for all $i \neq j$.

Theorem 1.11 ([5] Theorem 7.16). The Helmholtz equation is orthogonally separable if and only if there exist $n$ pointwise independent Killing tensors $\left(\mathbf{K}_{i}\right)$ one other commuting as linear operators, in involution and commuting with the Ricci tensor (Robertson condition).

As it is shown in [5, 6, 44] and recalled in [3] there exist some equivalent reformulations of Theorem 1.10 We first recall the definition of normal vector field.

Definition 1.5. On a riemannian manifold $\mathcal{M}$ a vector field $\mathbf{X}$ is called normal if it is orthogonally integrable or surface forming, i.e. if it is orthogonal to a foliation of $\mathcal{M}$ by hypersurfaces.

Theorem 1.12 (44, 4, 3]). The geodesic Hamilton-Jacobi equation is separable in orthogonal coordinates if and only if there exists a Killing 2-tensor with simple eigenvalues and normal eigenvectors. 
Definition 1.6. A Killing tensor having these properties is called a characteristic Killing tensor.

We can show that a characteristic Killing tensor generates a Killing-Stäckel space, i.e. a $n$-dimensional linear space $\mathcal{K}_{n}$ of Killing 2-tensors whose elements commute as linear operators and are in involution. Hence it is immediate from Theorem 1.12 that Theorem 1.10 holds.

We conclude this Subsection by an intrinsic characterization of separability for the Hamilton-Jacobi and the Helmholtz equations using symmetry operators given in [45]. We first recall the one-to-one correspondence between a second-order operator and a second order Killing tensor. We have already seen that the contravariant symmetric tensors are in one-to-one correspondence with the homogeneous polynomials on $T^{\star} \mathcal{M}$. Moreover, the operator $\hat{P}_{\mathbf{K}}$ corresponding to a second-degree homogeneous polynomial

$$
P_{\mathbf{K}}=K^{i j} p_{i} p_{j}
$$

associated with a symmetric contravariant two-tensor $\mathbf{K}$ on $\mathcal{M}$ is defined by

$$
\hat{P}_{\mathbf{K}} \psi=-\Delta_{\mathbf{K}} \psi=-\nabla_{i}\left(K^{i j} \nabla_{j} \psi\right) .
$$

We note that, if $\mathbf{K}=\mathbf{G}$ is the contravariant metric tensor, we obtain the Laplace-Beltrami operator $\Delta_{g}=\hat{P}_{\mathbf{G}}$.

Definition 1.7 (45]). We say that a second-order symmetry operator $\hat{P}$ is in self-adjoint form if $\hat{P}$ can be expressed into the form

$$
\hat{P}=\frac{1}{\sqrt{|g|}} \sum_{i, j} \partial_{i}\left(\sqrt{|g|} a^{i j} \partial_{j}\right)+c,
$$

where $|g|$ is the determinant of the metric, $a^{i j}=a^{j i}$, for all $(i, j) \in\{1, \ldots, n\}^{2}$, and $c$ is a real constant.

Remark 1.13. If $\hat{P}$ is a second-order selfadjoint operator we can associate to $\hat{P}$ the quadratic form on $T^{\star} \mathcal{M}$ defined by $P=a^{i j} p_{i} p_{j}$.

Theorem 1.14 (45] Theorem 3). Necessary and sufficient conditions for the existence of an orthogonal separable coordinate system $\left\{x^{i}\right\}$ for the Helmholtz equation are that there exists a linearly independent set $\left\{\hat{P}_{1}=\Delta_{g}, \hat{P}_{2}, \ldots, \hat{P}_{n}\right\}$ of second-order differential operators on $\mathcal{M}$ such that:

1. $\left[\hat{P}_{i}, \hat{P}_{j}\right]:=\hat{P}_{i} \hat{P}_{j}-\hat{P}_{j} \hat{P}_{i}=0$, for all $1 \leq i, j \leq n$.

2. Each $\hat{P}_{i}$ is in self-adjoint form.

3. There is a basis $\left\{\omega_{(j)}, 1 \leq j \leq n\right\}$ of simultaneous eigenforms for the $\left\{P_{i}\right\}$.

4. (Robertson condition) The associated second order Killing tensors $\left(\mathbf{K}_{i}\right)$ commute with the Ricci tensor.

If these conditions are satisfied then there exist functions $g^{i}(x)$ such that $\omega_{(j)} g^{j} d x^{j}$, for $j=1, \ldots, n$.

In our work we will give explicitly the operators $\hat{P}_{1}=\Delta_{g}, \hat{P}_{2}$ and $\hat{P}_{3}$ corresponding to our study. We finally note that there exists a more general notion of separability called the R-separation (see for instance [45, 5, 6]). Our notion of separability corresponds to the case $R=1$. The study of the R-separability in our framework will be the object of a future work. 


\subsection{Description of the framework}

The aim of this Subsection is to introduce the framework of our paper. Precisely, we give the definition of the Stäckel manifolds we are dealing with and we show that we can make, without loss of generality, some assumptions on the Stäckel manifolds we consider.

We start this Subsection by the description of the manifolds we study and the definition of the Stäckel structure. We first emphasize that the description given by Stäckel is local. We obtain a global description by choosing a global Stäckel system of coordinates which justify the name "Stäckel manifold". We thus consider manifolds with two ends having the topology of toric cylinders with a global chart

$$
\mathcal{M}=(0, A)_{x^{1}} \times \mathcal{T}_{x^{2}, x^{3}}^{2},
$$

where $\mathcal{T}_{x^{2}, x^{3}}^{2}=: \mathcal{T}^{2}$ denotes the 2 -dimensional torus. The variable $x^{1}$ is the radial variable whereas $\left(x^{2}, x^{3}\right)$ denotes the angular variables. We emphasize that we choose to work with angular variables living in a 2 -torus but it is also possible to choose other topologies. For instance the case of angular variables living in a 2 -sphere could be the object of a future work. We define a Stäckel matrix which is a $3 \times 3$ matrix of the form

$$
S=\left(\begin{array}{lll}
s_{11}\left(x^{1}\right) & s_{12}\left(x^{1}\right) & s_{13}\left(x^{1}\right) \\
s_{21}\left(x^{2}\right) & s_{22}\left(x^{2}\right) & s_{23}\left(x^{2}\right) \\
s_{31}\left(x^{3}\right) & s_{32}\left(x^{3}\right) & s_{33}\left(x^{3}\right)
\end{array}\right),
$$

where the coefficients $s_{i j}$ are smooth functions. Let $\mathcal{M}$ be endowed with the riemannian metric

$$
g=H_{1}^{2}\left(d x^{1}\right)^{2}+H_{2}^{2}\left(d x^{2}\right)^{2}+H_{3}^{2}\left(d x^{3}\right)^{2},
$$

with

$$
H_{i}^{2}=\frac{\operatorname{det}(S)}{s^{i 1}}, \quad \forall i \in\{1,2,3\},
$$

where $s^{i 1}$ is the minor (with sign) associated with the coefficient $s_{i 1}$ for all $i \in\{1,2,3\}$.

Remark 1.15. The metric $g$ is riemannian if and only if the determinant of the Stäckel matrix $S$ and the minors $s^{11}, s^{21}$ and $s^{31}$ have the same sign. Moreover, if we develop the determinant with respect to the first column, we note that if we assume that $s_{11}, s_{21}$ and $s_{31}$ are positive functions and if the minors $s^{11}, s^{21}$ and $s^{31}$ have the same sign, then the sign of the determinant of $S$ is necessary the same as the sign of these minors.

We emphasize that the application $S \mapsto g$ is not one-to-one. Indeed, we describe here two invariances of the metric which will be useful in the resolution of our inverse problem.

Proposition 1.16 (Invariances of the metric). Let $S$ be a Stäckel matrix.

1. Let $G$ be a $2 \times 2$ constant invertible matrix. The Stäckel matrix

$$
\hat{S}=\left(\begin{array}{lll}
s_{11}\left(x^{1}\right) & \hat{s}_{12}\left(x^{1}\right) & \hat{s}_{13}\left(x^{1}\right) \\
s_{21}\left(x^{2}\right) & \hat{s}_{22}\left(x^{2}\right) & \hat{s}_{23}\left(x^{2}\right) \\
s_{31}\left(x^{3}\right) & \hat{s}_{32}\left(x^{3}\right) & \hat{s}_{33}\left(x^{3}\right)
\end{array}\right),
$$

satisfying

$$
\left(\begin{array}{ll}
s_{i 2} & s_{i 3}
\end{array}\right)=\left(\hat{s}_{i 2} \quad \hat{s}_{i 3}\right) G, \quad \forall i \in\{1,2,3\},
$$

leads to the same metric as $S$. 
2. The Stäckel matrix

$$
\hat{S}=\left(\begin{array}{lll}
\hat{s}_{11}\left(x^{1}\right) & s_{12}\left(x^{1}\right) & s_{13}\left(x^{1}\right) \\
\hat{s}_{21}\left(x^{2}\right) & s_{22}\left(x^{2}\right) & s_{23}\left(x^{2}\right) \\
\hat{s}_{31}\left(x^{3}\right) & s_{32}\left(x^{3}\right) & s_{33}\left(x^{3}\right)
\end{array}\right)
$$

where,

$$
\left\{\begin{array}{l}
\hat{s}_{11}\left(x^{1}\right)=s_{11}\left(x^{1}\right)+C_{1} s_{12}\left(x^{1}\right)+C_{2} s_{13}\left(x^{1}\right) \\
\hat{s}_{21}\left(x^{2}\right)=s_{21}\left(x^{2}\right)+C_{1} s_{22}\left(x^{2}\right)+C_{2} s_{23}\left(x^{2}\right) \\
\hat{s}_{31}\left(x^{3}\right)=s_{31}\left(x^{3}\right)+C_{1} s_{32}\left(x^{3}\right)+C_{2} s_{33}\left(x^{3}\right)
\end{array},\right.
$$

where $C_{1}$ and $C_{2}$ are real constants, leads to the same metric as $S$.

Proof. We recall that

$$
g=\sum_{i=1}^{3} H_{i}^{2}\left(d x^{i}\right)^{2} \quad \text { with } \quad H_{i}^{2}=\frac{\operatorname{det}(S)}{s^{i 1}} \quad \forall i \in\{1,2,3\} .
$$

1. The result is an easy consequence of the equalities,

$$
s^{i 1}=\operatorname{det}(G) \hat{s}^{i 1}, \quad \forall i \in\{1,2,3\}, \quad \text { and } \quad \operatorname{det}(S)=\operatorname{det}(G) \operatorname{det}(\hat{S}) .
$$

2. The result follows from,

$$
s^{i 1}=\hat{s}^{i 1}, \quad \forall i \in\{1,2,3\}, \quad \text { and } \quad \operatorname{det}(S)=\operatorname{det}(\hat{S}) .
$$

Since we are only interested in recovering the metric $g$ of the Stäckel manifold, we can choose any representative of the equivalence class described by the invariances given in the previous Proposition. This fact allows us to make some assumptions on the Stäckel matrix we consider as we can see in the following Proposition.

Proposition 1.17. Let $S$ be a Stäckel matrix with corresponding metric $g_{S}$. There exists a Stäckel matrix $\hat{S}$ with $g_{\hat{S}}=g_{S}$ and such that

$$
\left\{\begin{array}{lll}
\hat{s}_{12}\left(x^{1}\right)>0 \quad \text { and } & \hat{s}_{13}\left(x^{1}\right)>0, & \forall x^{1} \\
\hat{s}_{22}\left(x^{2}\right)<0 \quad \text { and } \quad \hat{s}_{23}\left(x^{2}\right)>0, & \forall x^{2} \\
\hat{s}_{32}\left(x^{3}\right)>0 \quad \text { and } \quad \hat{s}_{33}\left(x^{3}\right)<0, & \forall x^{3} \\
\lim _{x^{1} \rightarrow 0} s_{12}\left(x^{1}\right)=\lim _{x^{1} \rightarrow 0} s_{13}\left(x^{1}\right)=1 &
\end{array} .\right.
$$

Proof. See Appendix A

Remark 1.18. The condition (C) has some interesting consequences which will be useful in our later analysis.

1. We note that under the condition (C), $s^{21}=s_{13} s_{32}-s_{12} s_{33}$ and $s^{31}=s_{12} s_{23}-s_{13} s_{22}$ are strictly positive. Thus, since the metric $g$ has to be a riemannian metric we must also have $\operatorname{det}(S)>0$ and $s^{11}>0$. 
2. We note that, since $s_{22}, s_{33}<0$ and $s_{23}, s_{32}>0$,

$$
s^{11}>0 \quad \Leftrightarrow \quad s_{22} s_{33}>s_{23} s_{32} \quad \Leftrightarrow \quad \frac{s_{22}}{s_{23}}<\frac{s_{32}}{s_{33}} .
$$

We will use these facts later in the study of the coupled spectrum of the operators $H$ and $L$ corresponding to the symmetry operators of $\Delta_{g}$ introduced in Subsection 1.1 .

From now on and without loss of generality, we assume that the Stäckel matrix $S$ we consider satisfies the condition (C).

On the Stäckel manifold $(\mathcal{M}, g)$ we are interested in studying of the Helmholtz equation

$$
-\Delta_{g} f=-\lambda^{2} f
$$

As mentionned in Subsection 1.1 the Stäckel structure is not enough to obtain the multiplicative separability of the Helmholtz equation. Indeed, we have to assume that the Robertson condition is satisfied. We recall that this condition can be defined as follows: for all $i \in\{1,2,3\}$ there exists $f_{i}\left(x^{i}\right)$, function of $x^{i}$ alone, such that

$$
\frac{s^{11} s^{21} s^{31}}{\operatorname{det}(S)}=f_{1} f_{2} f_{3}
$$

We can easily reformulate this condition into the form

$$
\frac{\operatorname{det}(S)^{2}}{H_{1}^{2} H_{2}^{2} H_{3}^{2}}=f_{1} f_{2} f_{3}
$$

Remark 1.19. We note that the functions $f_{i}, i \in\{1,2,3\}$, are defined up to positive multiplicative constants whose product is equal to one. In the following we will choose, without loss of generality, these constants equal to one.

\subsection{Asymptotically hyperbolic structure and examples}

The aim of this Subsection is to define the asymptotically hyperbolic structure we add on the previously defined Stäckel manifolds and to give three examples which illustrate the diversity of the manifolds we consider.

We say that a riemannian manifold $(\mathcal{M}, g)$ with boundary $\partial \mathcal{M}$ is asymptotically hyperbolic if its sectional curvatures tends to -1 at the boundary. In this paper, we put an asymptotically hyperbolic structure at the two radial ends of our Stäckel cylinders in the sense given by Isozaki and Kurylev in [40] (Section 3.2) We give now the definition of this structure in our framework.

Definition 1.8 (Asymptotically hyperbolic Stäckel manifold). A Stäckel manifold with two asymptotically hyperbolic ends having the topology of a toric cylinder is a Stäckel manifold $(\mathcal{M}, g)$ whose Stäckel matrix $S$ satisfies the condition (C) with a global chart

$$
\mathcal{M}=(0, A)_{x^{1}} \times \mathcal{T}_{x^{2}, x^{3}}^{2},
$$

where $x^{1} \in(0, A)_{x^{1}}$ corresponds to a boundary defining function for the two asymptotically hyperbolic ends $\left\{x^{1}=0\right\}$ and $\left\{x^{1}=A\right\}$ and $\left(x^{2}, x^{3}\right) \in[0, B]_{x^{2}} \times[0, C]_{x^{3}}$ are angular variables on the 2 -torus $\mathcal{T}_{x^{2}, x^{3}}^{2}$, satisfying the following conditions.

\footnotetext{
${ }^{1}$ Note that the asymptotically hyperbolic structure introduced in 40, is slightly more general than the one used by Melrose, Guillarmou, Joshi and Sá Barreto in [35, 42, 58, 65.
} 
1. The Stäckel metric g has the form 1.4.).

2. The coefficients $s_{i j},(i, j) \in\{1,2,3\}^{2}$, of the Stäckel matrix are smooth functions.

3. The coefficients of the Stäckel matrix satisfy:

(a) $H_{i}^{2}>0$ for $i \in\{1,2,3\}$ (riemannian metric).

(b) $s_{2 j}(0)=s_{2 j}(B), s_{2 j}^{\prime}(0)=s_{2 j}^{\prime}(B), s_{3 j}(0)=s_{3 j}(C)$ and $s_{3 j}^{\prime}(0)=s_{3 j}^{\prime}(C)$ for $j \in\{1,2,3\}$ (Periodic conditions in angular variables).

(c) Asymptotically hyperbolic ends at $\left\{x^{1}=0\right\}$ and $\left\{x^{1}=A\right\}$ :

i. At $\left\{x^{1}=0\right\}$ : there exist $\epsilon_{0}>0$ and $\delta>0$ such that $\forall n \in \mathbb{N}$ there exists $C_{n}>0$ such that: $\forall x^{1} \in(0, A-\delta)$,

$$
\begin{gathered}
\left\|\left(x^{1} \partial_{x^{1}}\right)^{n}\left(\left(x^{1}\right)^{2} s_{11}\left(x^{1}\right)-1\right)\right\| \leq C_{n}\left(1+\left|\log \left(x^{1}\right)\right|\right)^{-\min (n, 1)-1-\epsilon_{0}}, \\
\left\|\left(x^{1} \partial_{x^{1}}\right)^{n}\left(s_{12}\left(x^{1}\right)-1\right)\right\| \leq C_{n}\left(1+\left|\log \left(x^{1}\right)\right|\right)^{-\min (n, 1)-1-\epsilon_{0}} \\
\left\|\left(x^{1} \partial_{x^{1}}\right)^{n}\left(s_{13}\left(x^{1}\right)-1\right)\right\| \leq C_{n}\left(1+\left|\log \left(x^{1}\right)\right|\right)^{-\min (n, 1)-1-\epsilon_{0}} .
\end{gathered}
$$

ii. At $\left\{x^{1}=A\right\}$ : there exist $\epsilon_{1}>0$ and $\delta>0$ such that $\forall n \in \mathbb{N}$ there exists $C_{n}>0$ such that: $\forall x^{1} \in(\delta, A)$,

$$
\begin{gathered}
\left\|\left(\left(A-x^{1}\right) \partial_{x^{1}}\right)^{n}\left(\left(A-x^{1}\right)^{2} s_{11}\left(x^{1}\right)-1\right)\right\| \leq C_{n}\left(1+\left|\log \left(\left(A-x^{1}\right)\right)\right|\right)^{-\min (n, 1)-1-\epsilon_{1}}, \\
\left\|\left(\left(A-x^{1}\right) \partial_{x^{1}}\right)^{n}\left(s_{12}\left(x^{1}\right)-1\right)\right\| \leq C_{n}\left(1+\left|\log \left(\left(A-x^{1}\right)\right)\right|\right)^{-\min (n, 1)-1-\epsilon_{1}} \\
\left\|\left(\left(A-x^{1}\right) \partial_{x^{1}}\right)^{n}\left(s_{13}\left(x^{1}\right)-1\right)\right\| \leq C_{n}\left(1+\left|\log \left(\left(A-x^{1}\right)\right)\right|\right)^{-\min (n, 1)-1-\epsilon_{1}} .
\end{gathered}
$$

Remark 1.20. We know that, thanks to the condition (C), $s_{12}$ and $s_{13}$ tend to 1 when $x^{1}$ tends to 0 . However, at the end $\left\{x^{1}=A\right\}$, we can just say that there exist two positive constants $\alpha$ and $\beta$ such that $s_{12}$ and $s_{13}$ tend to $\alpha$ and $\beta$ respectively. Thus, at the end $\left\{x^{1}=A\right\}$, we should assume that

$$
\left(A-x^{1}\right)^{2} s_{11}\left(x^{1}\right)=[1]_{\epsilon_{1}}, \quad s_{12}\left(x^{1}\right)=\alpha[1]_{\epsilon_{1}} \quad \text { and } \quad s_{13}\left(x^{1}\right)=\beta[1]_{\epsilon_{1}},
$$

where,

$$
[1]_{\epsilon_{1}}=1+O\left(\left(1+\left|\log \left(A-x^{1}\right)\right|\right)^{-1-\epsilon_{1}}\right) .
$$

However, we can show (see the last point of Remark 1.22) that, if $s_{22}$ or $s_{33}$ are not constant functions, then $\alpha=\beta=1$.

Let us explain the meaning of asymptotically hyperbolic ends for Stäckel manifolds we put her£ ${ }^{2}$. Since the explanation is similar at the end $\left\{x^{1}=A\right\}$ we just study the end $\left\{x^{1}=0\right\}$. We first write the metric (1.4) in a neighbourhood of $\left\{x^{1}=0\right\}$ into the form

$$
g=\sum_{i=1}^{3} H_{i}^{2}\left(d x^{i}\right)^{2}=\frac{\sum_{i=1}^{3}\left(x^{1}\right)^{2} H_{i}^{2}\left(d x^{i}\right)^{2}}{\left(x^{1}\right)^{2}} .
$$

\footnotetext{
${ }^{2}$ We refer to [40], Section 3 p.99-101, for a justification of the name "asymptotically hyperbolic".
} 
By definition,

$$
\left\{\begin{array}{l}
\left(x^{1}\right)^{2} H_{1}^{2}=\left(x^{1}\right)^{2} s_{11}+\left(x^{1}\right)^{2}\left(s_{12} \frac{s^{12}}{s^{11}}+s_{13} \frac{s^{13}}{s^{21}}\right) \\
\left(x^{1}\right)^{2} H_{2}^{2}=\left(x^{1}\right)^{2} s_{11} \frac{s^{11}}{s_{32} s_{13}-s_{33} s_{12}}+\left(x^{1}\right)^{2}\left(s_{12} \frac{s^{12}}{s_{32} s_{13}-s_{33} s_{12}}+s_{13} \frac{s^{13}}{s_{32} s_{13}-s_{33} s_{12}}\right) \\
\left(x^{1}\right)^{2} H_{3}^{2}=\left(x^{1}\right)^{2} s_{11} \frac{s^{11}}{s_{23} s_{12}-s_{22} s_{13}}+\left(x^{1}\right)^{2}\left(\frac{s^{12}}{s_{23} s_{12}-s_{22} s_{13}}+\frac{s_{13}}{s_{12}} \frac{s^{13}}{s_{23} s_{12}-s_{22} s_{13}}\right)
\end{array} .\right.
$$

As it is shown in [57, we know that at the end $\left\{x^{1}=0\right\}$, the sectional curvature of $g$ approaches $-\left|d x^{1}\right|_{h}$ where

$$
h=\sum_{i=1}^{3}\left(x^{1}\right)^{2} H_{i}^{2}\left(d x^{i}\right)^{2} .
$$

In other words, the opposite of the sectional curvature at the end $\left\{x^{1}=0\right\}$ is equivalent to

$$
\left(x^{1}\right)^{2} H_{1}^{2}=\left(x^{1}\right)^{2} s_{11}+\left(x^{1}\right)^{2}\left(s_{12} \frac{s^{12}}{s^{11}}+s_{13} \frac{s^{13}}{s^{21}}\right) .
$$

Thus, since an asymptotically hyperbolic structure corresponds to a sectional curvature which tends to -1 , we want that this last quantity tends to 1 . This is ensured by the third assumption of Definition 1.8 which entails that (for $n=0$ ):

$$
\left(x^{1}\right)^{2} s_{11}\left(x^{1}\right)=[1]_{\epsilon_{0}}, \quad s_{12}\left(x^{1}\right)=[1]_{\epsilon_{0}} \quad \text { and } \quad s_{13}\left(x^{1}\right)=[1]_{\epsilon_{0}},
$$

where

$$
[1]_{\epsilon_{0}}=1+O\left(\left(1+\left|\log \left(x^{1}\right)\right|\right)^{-1-\epsilon_{0}}\right) .
$$

We also note that, under these conditions we can write thanks to 1.8 the metric $g$, in a neighbourhood of $\left\{x^{1}=0\right\}$, into the form

$$
g=\frac{\left(d x^{1}\right)^{2}+d \Omega_{\mathcal{T}^{2}}^{2}+P\left(x^{1}, x^{2}, x^{3}, d x^{1}, d x^{2}, d x^{3}\right)}{\left(x^{1}\right)^{2}},
$$

where

$$
d \Omega_{\mathcal{T}^{2}}^{2}=\frac{s^{11}}{s_{32}-s_{33}}\left(d x^{2}\right)^{2}+\frac{s^{11}}{s_{23}-s_{22}}\left(d x^{3}\right)^{2},
$$

is a riemannian metric on the 2-torus $\mathcal{T}^{2}$ (since $s^{11}, s^{21}$ and $s^{31}$ have the same sign) and $P$ is a remainder term which is, roughly speaking, small as $x^{1} \rightarrow 0$. Hence, in the limit $x^{1} \rightarrow 0$, we see that

$$
g \sim \frac{\left(d x^{1}\right)^{2}+d \Omega_{\mathcal{T}^{2}}^{2}}{\left(x^{1}\right)^{2}}
$$

that is, $g$ is a small perturbation of a hyperbolic like metric.

Remark 1.21. 1. According to the previous definition, we also need conditions on the derivatives of $s_{1 j}, j \in\{1,2,3\}$, to be in the framework of [40].

2. By symmetry, we can do the same analysis at the end $\left\{x^{1}=A\right\}$. 
From the conditions $(1.9)$ and the Robertson condition 1.6 we can obtain more informations on the functions $f_{1}, f_{2}$ and $f_{3}$. We first remark that

$$
\begin{aligned}
f_{1} f_{2} f_{3} & =\frac{s^{11} s^{21} s^{31}}{\operatorname{det}(S)} \\
& =\frac{s^{11}\left(s_{13} s_{32}-s_{12} s_{33}\right)\left(s_{12} s_{23}-s_{13} s_{22}\right)}{s_{11} s^{11}+s_{12} s^{12}+s_{13} s^{13}} .
\end{aligned}
$$

Thus, using the conditions 1.9 , we obtain

$$
f_{1} f_{2} f_{3} \sim\left(x^{1}\right)^{2}\left(s_{23}-s_{22}\right)\left(s_{32}-s_{33}\right), \quad \text { when } \quad x^{1} \rightarrow 0 .
$$

Hence, we can say that there exist three positive constants $c_{1}, c_{2}$ and $c_{3}$ such that $c_{1} c_{2} c_{3}=1$ and

$$
f_{1}\left(x^{1}\right)=c_{1}\left(x^{1}\right)^{2}[1]_{\epsilon_{0}}, \quad f_{2}\left(x^{2}\right)=c_{2}\left(s_{23}-s_{22}\right) \quad \text { and } \quad f_{3}\left(x^{3}\right)=c_{3}\left(s_{32}-s_{33}\right) .
$$

We thus note that the functions $f_{i}, i \in\{1,2,3\}$, are defined up to positive constants $c_{1}, c_{2}$ and $c_{3}$ whose product is equal to 1 . However, as mentioned previously, we can choose these constants as equal to 1 . Of course, the corresponding result on $f_{1}$ at the end $\left\{x^{1}=A\right\}$ is also true.

Remark 1.22. The previous analysis allows us to simplify the Robertson condition and thus the expression of the riemannian metric on the 2-torus.

1. We first note that, if we make a Liouville change of variables in the $i^{\text {th }}$-variable,

$$
X^{i}=\int_{0}^{x^{i}} \sqrt{g_{i}(s)} d s
$$

where $g_{i}$ is a positive function of the variable $x^{i}$, the corresponding coefficient $H_{i}^{2}$ of the metric is also divided by $g_{i}\left(x^{i}\right)$. The same modification of the metric happens when we divide the $i^{\text {th }}$-line of the Stäckel matrix by the function $g_{i}$. Thus, to proceed to a Liouville change of variables is equivalent to divide the $i$-th line of the Stäckel matrix by the corresponding function.

2. We now remark that, if we divide the $i^{\text {th }}$-line of the Stäckel matrix by a function $g_{i}$ of the variable $x^{i}$, the quantity

$$
\frac{s^{11} s^{21} s^{31}}{\operatorname{det}(S)}
$$

is divided by $g_{i}$. Thus, recalling the form of the Robertson condition (1.6), we can always assume that $f_{2}=f_{3}=1$ by choosing appropriate coordinates on $\mathcal{T}^{2}$. However, we do not divide the first line by $f_{1}$ because it changes the description of the hyperbolic structure (i.e. the condition (1.9)). Nevertheless, it remains a degree of freedom on the first line. For instance, we can divide the first line by $s_{12}$ or $s_{13}$ and we then obtain that the radial part depends only on the two scalar functions $\frac{s_{11}}{s_{13}}$ and $\frac{s_{12}}{s_{13}}$. As we will see at the end of Section 4 , these quotients are exactly the scalar functions we recover in our study of the radial part. However, since it does not simplify our study we do not use this reduction for the moment.

3. From now on, $f_{2}=1$ and $f_{3}=1$ and we can thus rewrite (1.11) into the form

$$
s_{23}-s_{22}=1 \quad \text { and } \quad s_{32}-s_{33}=1 .
$$


Thanks to these equalities, we can also write

$$
d \Omega_{\mathcal{T}^{2}}^{2}=s^{11}\left(\left(d x^{2}\right)^{2}+\left(d x^{3}\right)^{2}\right),
$$

for the induced metric on the compactified boundary $\left\{x^{1}=0\right\}$.

4. Generally, we know that $s_{12}$ and $s_{13}$ tend to 1 when $x^{1}$ tends to 0 but we do not know that this is also true when $x^{1}$ tends to A. However, the Stäckel structure allows us to show that the asymptotically hyperbolic structure has to be the same at the two ends (under a mild additional assumption). Assume that the behaviour of the first line at the two ends is the following: at the end $\left\{x^{1}=0\right\}$

$$
\left(x^{1}\right)^{2} s_{11}\left(x^{1}\right)=[1]_{\epsilon_{0}}, \quad s_{12}\left(x^{1}\right)=[1]_{\epsilon_{0}} \quad \text { and } \quad s_{13}\left(x^{1}\right)=[1]_{\epsilon_{0}},
$$

and at the end $\left\{x^{1}=A\right\}$

$$
\left(A-x^{1}\right)^{2} s_{11}\left(x^{1}\right)=[1]_{\epsilon_{1}}, \quad s_{12}\left(x^{1}\right)=\alpha[1]_{\epsilon_{1}} \quad \text { and } \quad s_{13}\left(x^{1}\right)=\beta[1]_{\epsilon_{1}},
$$

where,

$$
[1]_{\epsilon_{0}}=1+O\left(\left(1+\left|\log \left(x^{1}\right)\right|\right)^{-1-\epsilon_{0}}\right) \quad \text { and } \quad[1]_{\epsilon_{1}}=1+O\left(\left(1+\left|\log \left(A-x^{1}\right)\right|\right)^{-1-\epsilon_{1}}\right)
$$

and $\alpha$ and $\beta$ are real positive constants. Using the Robertson condition at the end $\left\{x^{1}=0\right\}$ and the end $\left\{x^{1}=A\right\}$ we obtain

$$
1=f_{2}=s_{23}-s_{22}=\alpha s_{23}-\beta s_{22},
$$

and

$$
1=f_{3}=s_{32}-s_{33}=\beta s_{32}-\alpha s_{33} .
$$

Thus, using that $s_{23}=1+s_{22}$ and $s_{32}=1+s_{33}$, we obtain

$$
(\alpha-\beta) s_{22}=1-\alpha \quad \text { and } \quad(\beta-\alpha) s_{33}=1-\beta .
$$

Hence, if we assume that $s_{22}$ or $s_{33}$ are not constant functions, we obtain $\alpha=\beta=1$.

Finally, we now give three examples of Stäckel manifolds.

Example 1.2. We give here three examples of Stäckel manifolds which illustrate the diversity of the manifolds we consider.

1. We can first choose the Stäckel matrix

$$
S=\left(\begin{array}{ccc}
s_{11}\left(x^{1}\right) & s_{12}\left(x^{1}\right) & s_{13}\left(x^{1}\right) \\
a & b & c \\
d & e & f
\end{array}\right),
$$

where $s_{11}, s_{12}$ and $s_{13}$ are smooth functions of $x^{1}$ and $a, b, c, d, e$ and $f$ are real constants. The metric $g$ can thus be written as

$$
g=\sum_{i=1}^{3} H_{i}^{2}\left(d x^{i}\right)^{2},
$$

where $H_{i}^{2}$, for $i \in\{1,2,3\}$, are functions of $x^{1}$ alone. Therefore, $g$ trivially satisfies the Robertson condition and we can add the asymptotically hyperbolic structure given in Definition 1.8. We note that, as explained in the previous Remark, $g$ depends only on two arbitrary functions (after a Liouville change of variables in the variable $\left.x^{1}\right)$. Moreover, we can show that $\partial_{x^{2}}$ and $\partial_{x^{3}}$ are Killing vector fields and the existence of these Killing vector fields traduces the symmetries with respect to the translation in $x^{2}$ and $x^{3}$. 
2. We can also choose the Stäckel matrix

$$
S=\left(\begin{array}{ccc}
s_{11}\left(x^{1}\right) & s_{12}\left(x^{1}\right) & a s_{12}\left(x^{1}\right) \\
0 & s_{22}\left(x^{2}\right) & s_{23}\left(x^{2}\right) \\
0 & s_{32}\left(x^{3}\right) & s_{33}\left(x^{3}\right)
\end{array}\right)
$$

where $s_{11}$ and $s_{12}$ are smooth functions of $x^{1}, s_{22}$ and $s_{23}$ are smooth functions of $x^{2}$, $s_{32}$ and $s_{33}$ are smooth functions of $x^{3}$ and $a$ is a real constant. We can add the asymptotically hyperbolic structure given in Definition 1.8 and the metric $g$ can be written as

$$
g=s_{11}\left(d x^{1}\right)^{2}+\frac{s_{11}}{s_{12}}\left(\frac{s^{11}}{a s_{32}-s_{33}}\left(d x^{2}\right)^{2}+\frac{s^{11}}{s_{23}-a s_{22}}\left(d x^{3}\right)^{2}\right) .
$$

Therefore, $g$ satisfied the Robertson condition. We note that, after Liouville transformations in the three variables, $g$ depends on three arbitrary functions. Moreover, thanks to the Liouville transformation

$$
X^{1}=\int_{0}^{x^{1}} \sqrt{s_{11}(s)} d s
$$

we see that there exists a system of coordinates in which the metric $g$ takes the form

$$
g=\left(d x^{1}\right)^{2}+f\left(x^{1}\right) g_{0},
$$

where $g_{0}$ is a metric on the 2-torus $\mathcal{T}^{2}$. In other words, $g$ is a warped product. In particular, $g$ is conformal to a metric which can be written as a sum of one euclidean direction and a metric on a compact manifold. We recall that in this case, under some additional assumptions on the compact part, the uniqueness of the anisotropic Calderón problem on compact manifolds with boundary has been proved in [27, 28].

3. At last, we can choose the Stäckel matrix

$$
S=\left(\begin{array}{ccc}
s_{1}\left(x^{1}\right)^{2} & -s_{1}\left(x^{1}\right) & 1 \\
-s_{2}\left(x^{2}\right)^{2} & s_{2}\left(x^{2}\right) & -1 \\
s_{3}\left(x^{3}\right)^{2} & -s_{3}\left(x^{3}\right) & 1
\end{array}\right),
$$

where $s_{1}$ is a smooth function of $x^{1}, s_{2}$ is a smooth function of $x^{2}$ and $s_{3}$ is a smooth function of $x^{3}$. This model was studied in [3, 12] and is of main interest in the field of geodesically equivalent riemannian manifolds i.e. of manifold which share the same unparametrized geodesics (see [12]). The associated metric

$$
g=\left(s_{1}-s_{2}\right)\left(s_{1}-s_{3}\right)\left(d x^{1}\right)^{2}+\left(s_{2}-s_{3}\right)\left(s_{1}-s_{2}\right)\left(d x^{2}\right)^{2}+\left(s_{3}-s_{2}\right)\left(s_{3}-s_{1}\right)\left(d x^{3}\right)^{2},
$$

satisfies the Robertson condition and $g$ has, a priori, no symmetry, is not a warped product and depends on three arbitrary functions that satisfy $s_{1}>s_{2}>s_{3}$. To put an asymptotically hyperbolic structure in the sense given in Definition 1.8 we first multiply the second and the third column of the Stäckel matrix on the right by the invertible matrix

$$
G=\left(\begin{array}{cc}
-1 & -1 \\
0 & -1
\end{array}\right)
$$


since it does not change the metric. We thus obtain the new Stäckel matrix

$$
\left(\begin{array}{ccc}
s_{1}\left(x^{1}\right)^{2} & s_{1}\left(x^{1}\right) & s_{1}\left(x^{1}\right)-1 \\
-s_{2}\left(x^{2}\right)^{2} & -s_{2}\left(x^{2}\right) & -s_{2}\left(x^{1}\right)+1 \\
s_{3}\left(x^{3}\right)^{2} & s_{3}\left(x^{3}\right) & s_{3}\left(x^{1}\right)-1
\end{array}\right) .
$$

In a second time, we use the Liouville change of variables in the first variable

$$
X^{1}=\int_{0}^{x^{1}} \sqrt{s_{1}(s)} d s,
$$

and we obtain the Stäckel matrix

$$
S=\left(\begin{array}{ccc}
s_{1}\left(X^{1}\right) & 1 & 1-\frac{1}{s_{1}\left(X^{1}\right)} \\
-s_{2}\left(x^{2}\right)^{2} & -s_{2}\left(x^{2}\right) & -s_{2}\left(x^{1}\right)+1 \\
s_{3}\left(x^{3}\right)^{2} & s_{3}\left(x^{3}\right) & s_{3}\left(x^{1}\right)-1
\end{array}\right)
$$

Finally, to put the asymptotically hyperbolic structure on the first line, we assume that

$$
s_{1}\left(X^{1}\right)=\frac{1}{\left(X^{1}\right)^{2}}\left(1+O\left(\left(1+\left|\log \left(X^{1}\right)\right|\right)^{-1-\epsilon_{0}}\right)\right), \quad \text { when } \quad X^{1} \rightarrow 0
$$

and

$$
s_{1}\left(X^{1}\right)=\frac{1}{\left(A^{1}-X^{1}\right)^{2}}\left(1+O\left(\left(1+\left|\log \left(A^{1}-X^{1}\right)\right|\right)^{-1-\epsilon_{1}}\right)\right), \quad \text { when } \quad X^{1} \rightarrow A^{1},
$$

where $A^{1}=\int_{0}^{A} \sqrt{s_{1}(s)} d s$.

\subsection{Scattering operator and statement of the main result}

We recall here the construction of the scattering operator given in [40, 41] for asymptotically hyperbolic manifolds. This construction has been used in [22] in the case of asymptotically hyperbolic Liouville surfaces. Roughly speaking, in the neighbourhood of the asymptotically hyperbolic ends we can compare the global dynamics with a simpler comparison dynamics, i.e. we establish the existence and the asymptotically completeness of the wave operators

$$
W_{k}^{ \pm}=s-\lim e^{i t H} J_{k} e^{-i t H_{0}^{k}},
$$

where $J_{k}$ is a cutoff function that isolate the $k^{\text {th }}$ asymptotically hyperbolic end and $H_{0}^{k}$ is a simpler hamiltonian which governs the free wave dynamics in this end. The scattering operator $S_{g}$ is then defined by

$$
S_{g}=\left(W^{+}\right)^{\star} W^{-}, \quad \text { where } \quad W^{ \pm}=\sum_{k} W_{k}^{ \pm} .
$$

This operator makes the link between the asymptotic (scattering) data in the past and the asymptotic (scattering) data in the future. The scattering matrix $S_{g}(\lambda)$ is then the restriction of the scattering operator $S_{g}$ on an energy level $\lambda^{2}$. This corresponds to the time-dependent approach to scattering theory. There is also an equivalent stationary definition. To define the scattering matrix in a stationary way, we take the Fourier transform of the wave equation $\partial_{t}^{2} u-\Delta_{g} u=0$ with respect to $t$, and instead of studying the asymptotic behaviour of $u(t, x)$ at late times, we study the spatial asymptotic behaviour of 
solutions of the Helmholtz equation $-\Delta_{g} u=\lambda^{2} u$ as $|x| \rightarrow \infty$. We thus obtain an other but equivalent definition of the scattering matrix at energy $\lambda^{2}$ thanks to the Helmholtz equation (see Theorem 1.24).

In our particular model, there are two ends and so we introduce two cutoff functions $\chi_{0}$ and $\chi_{1}$, smooth on $\mathbb{R}$, defined by

$$
\chi_{0}=1 \quad \text { on } \quad\left(0, \frac{A}{4}\right), \quad \chi_{1}=1 \quad \text { on } \quad\left(\frac{3 A}{4}, A\right), \quad \chi_{0}+\chi_{1}=1 \quad \text { on } \quad(0, A),
$$

in order to separate these two ends. We consider the shifted stationary Helmholtz equation

$$
-\left(\Delta_{g}+1\right) f=\lambda^{2} f
$$

where $\lambda^{2} \neq 0$ is a fixed energy, which is usually studied in case of asymptotically hyperbolic manifolds (see 13, 40, 41, 42 ). Indeed, it is known (see 41]) that the essential spectrum of $-\Delta_{g}$ is $[1,+\infty$ ) and thus, we shift the bottom of the essential spectrum in order that it becomes 0 . It is known that the operator $-\Delta_{g}-1$ has no eigenvalues embedded into the essential spectrum $[0,+\infty)$ (see [15, 40, 41]). It is shown in [41] that the solutions of the shifted stationary equation

$$
-\left(\Delta_{g}+1\right) f=\lambda^{2} f
$$

are unique when we impose on $f$ some radiation conditions at infinities. Precisely, as in [22], we define some Besov spaces that encode these radiation conditions at infinities as follows. To motivate our definitions, we first recall that the compactified boundaries $\left\{x^{1}=0\right\}$ and $\left\{x^{1}=A\right\}$ are endowed with the induced metric

$$
d \Omega_{\mathcal{T}^{2}}^{2}=s^{11}\left(\left(d x^{2}\right)^{2}+\left(d x^{3}\right)^{2}\right) .
$$

Definition 1.9. Let $\mathcal{H}_{\mathcal{T}^{2}}=L^{2}\left(\mathcal{T}^{2}, s^{11} d x^{2} d x^{3}\right)$. Let the intervals $(0,+\infty)$ and $(-\infty, A)$ be decomposed as

$$
(0,+\infty)=\cup_{k \in \mathbb{Z}} I_{k} \quad \text { and } \quad(-\infty, A)=\cup_{k \in \mathbb{Z}} J_{k},
$$

where

$$
I_{k}=\left\{\begin{array}{l}
\left(\exp \left(e^{k-1}\right), \exp \left(e^{k}\right)\right] \quad \text { if } \quad k \geq 1 \\
\left(e^{-1}, e\right] \quad \text { if } \quad k=0 \\
\left(\exp \left(-e^{|k|}\right), \exp \left(-e^{|k|-1}\right)\right] \quad \text { if } \quad k \leq-1
\end{array}\right.
$$

and

$$
J_{k}=\left\{\begin{array}{l}
\left(A-\exp \left(e^{k-1}\right), A-\exp \left(e^{k}\right)\right] \quad \text { if } \quad k \geq 1 \\
\left(A-e^{-1}, A-e\right] \quad \text { if } \quad k=0 \\
\left(A-\exp \left(-e^{|k|}\right), A-\exp \left(-e^{|k|-1}\right)\right] \quad \text { if } \quad k \leq-1
\end{array} .\right.
$$

We define the Besov spaces $\mathcal{B}_{0}=\mathcal{B}_{0}\left(\mathcal{H}_{\mathcal{T}^{2}}\right)$ and $\mathcal{B}_{1}=\mathcal{B}_{1}\left(\mathcal{H}_{\mathcal{T}^{2}}\right)$ to be the Banach spaces of $\mathcal{H}_{\mathcal{T}^{2}}$-valued functions on $(0,+\infty)$ and $(-\infty, A)$ satisfying respectively

$$
\|f\|_{\mathcal{B}_{0}}=\sum_{k \in \mathbb{Z}} e^{\frac{|k|}{2}}\left(\int_{I_{k}}\|f(x)\|_{\mathcal{H}_{\mathcal{T}^{2}}}^{2} \frac{d x}{x^{2}}\right)^{\frac{1}{2}}<\infty
$$

and

$$
\|f\|_{\mathcal{B}_{1}}=\sum_{k \in \mathbb{Z}} e^{\frac{|k|}{2}}\left(\int_{J_{k}}\|f(x)\|_{\mathcal{H}_{\mathcal{T}^{2}}}^{2} \frac{d x}{(A-x)^{2}}\right)^{\frac{1}{2}}<\infty .
$$


The dual spaces $\mathcal{B}_{0}^{\star}$ and $\mathcal{B}_{1}^{\star}$ are then identified with the spaces equipped with the norms

$$
\|f\|_{\mathcal{B}_{0}^{\star}}=\left(\sup _{R>e} \frac{1}{\log (R)} \int_{\frac{1}{R}}^{R}\|f(x)\|_{\mathcal{H}_{\mathcal{T}^{2}}}^{2} \frac{d x}{x^{2}}\right)^{\frac{1}{2}}<\infty
$$

and

$$
\|f\|_{\mathcal{B}_{1}^{\star}}=\left(\sup _{R>e} \frac{1}{\log (R)} \int_{A-R}^{A-\frac{1}{R}}\|f(x)\|_{\mathcal{H}_{\mathcal{T}^{2}}}^{2} \frac{d x}{(A-x)^{2}}\right)^{\frac{1}{2}}<\infty .
$$

Remark 1.23. As shown in $[40]$, we can compare the Besov spaces $\mathcal{B}_{0}$ and $\mathcal{B}_{0}^{\star}$ to weighted $L^{2}$-spaces. Indeed, if we define $L_{0}^{2, s}\left((0,+\infty), \mathcal{H}_{\mathcal{T}^{2}}\right)$ for $s \in \mathbb{R}$ by

$$
\|f\|_{s}=\left(\int_{0}^{+\infty}(1+|\log (x)|)^{2 s}\|f(x)\|_{\mathcal{H}_{\mathcal{T}^{2}}}^{2} \frac{d x}{x^{2}}\right)^{\frac{1}{2}}<\infty
$$

then for $s>\frac{1}{2}$,

$$
L_{0}^{2, s} \subset \mathcal{B}_{0} \subset L_{0}^{2, \frac{1}{2}} \subset L_{0}^{2} \subset L_{0}^{2,-\frac{1}{2}} \subset \mathcal{B}_{0}^{\star} \subset L_{0}^{2,-s} .
$$

There is a similar result for the Besov spaces $\mathcal{B}_{1}$ and $\mathcal{B}_{1}^{\star}$.

Definition 1.10. We define the Besov spaces $\mathcal{B}$ and $\mathcal{B}^{\star}$ as the Banach spaces of $\mathcal{H}_{\mathcal{T}^{2} \text {-valued functions }}$ on $(0, A)$ with norms

$$
\|f\|_{\mathcal{B}}=\left\|\chi_{0} f\right\|_{\mathcal{B}_{0}}+\left\|\chi_{1} f\right\|_{\mathcal{B}_{1}}
$$

and

$$
\|f\|_{\mathcal{B}^{\star}}=\left\|\chi_{0} f\right\|_{\mathcal{B}_{0}^{\star}}+\left\|\chi_{1} f\right\|_{\mathcal{B}_{1}^{\star}} .
$$

We also define the Hilbert space of scattering data

$$
\mathcal{H}_{\infty}=\mathcal{H}_{\mathcal{T}^{2}} \otimes \mathbb{C}^{2} \simeq \mathcal{H}_{\mathcal{T}^{2}} \oplus \mathcal{H}_{\mathcal{T}^{2}}
$$

In 40, (see Theorem 3.15) the following theorem is proved.

Theorem 1.24 (Stationary construction of the scattering matrix). 1. For any solution $f \in \mathcal{B}^{\star}$ of the shifted stationary Helmholtz equation at non-zero energy $\lambda^{2}$

$$
-\left(\Delta_{g}+1\right) f=\lambda^{2} f
$$

there exists a unique $\psi^{( \pm)}=\left(\psi_{0}^{( \pm)}, \psi_{1}^{( \pm)}\right) \in \mathcal{H}_{\infty}$ such that

$$
\begin{aligned}
f \simeq & \omega_{-}(\lambda)\left(\chi_{0}\left(x^{1}\right)^{\frac{1}{2}+i \lambda} \psi_{0}^{(-)}+\chi_{1}\left(A-x^{1}\right)^{\frac{1}{2}+i \lambda} \psi_{1}^{(-)}\right) \\
& -\omega_{+}(\lambda)\left(\chi_{0}\left(x^{1}\right)^{\frac{1}{2}-i \lambda} \psi_{0}^{(+)}+\chi_{1}\left(A-x^{1}\right)^{\frac{1}{2}-i \lambda} \psi_{1}^{(+)}\right),
\end{aligned}
$$

where

$$
\omega_{ \pm}(\lambda)=\frac{\pi}{(2 \lambda \sinh (\pi \lambda))^{\frac{1}{2}} \Gamma(1 \mp i \lambda)} .
$$

2. For any $\psi^{(-)} \in \mathcal{H}_{\infty}$, there exists a unique $\psi^{(+)} \in \mathcal{H}_{\infty}$ and $f \in \mathcal{B}^{\star}$ satisfying 1.14) for which the decomposition (1.15) above holds. This defines uniquely the scattering operator $S_{g}(\lambda)$ as the $\mathcal{H}_{\infty}$-valued operator such that for all $\psi^{(-)} \in \mathcal{H}_{\infty}$,

$$
\psi^{(+)}=S_{g}(\lambda) \psi^{(-)} .
$$


3. The scattering operator $S_{g}(\lambda)$ is unitary on $\mathcal{H}_{\infty}$.

Note that in our model with two asymptotically hyperbolic ends the scattering operator has the structure of a $2 \times 2$ matrix whose components are $\mathcal{H}_{\mathcal{T}^{2} \text {-valued operators. Precisely, we write }}$

$$
S_{g}(\lambda)=\left(\begin{array}{cc}
L(\lambda) & T_{R}(\lambda) \\
T_{L}(\lambda) & R(\lambda)
\end{array}\right)
$$

where $T_{L}(\lambda)$ and $T_{R}(\lambda)$ are the transmission operators and $L(\lambda)$ and $R(\lambda)$ are the reflection operators from the right and from the left respectively. The transmission operators measure what is transmitted from one end to the other end in a scattering experiment, while the reflection operators measure the part of a signal sent from one end that is reflected to itself.

The main result of this paper is the following:

Theorem 1.25. Let $(\mathcal{M}, g)$ and $(\mathcal{M}, \tilde{g})$, where $\mathcal{M}=(0, A)_{x^{1}} \times \mathcal{T}_{x^{2}, x^{3}}^{2}$, be two three-dimensional Stäckel toric cylinders, i.e. endowed with the metrics $g$ and $\tilde{g}$ defined in (1.4) respectively. We assume that these manifolds satisfy the Robertson condition and are endowed with asymptotically hyperbolic structures at the two ends $\left\{x^{1}=0\right\}$ and $\left\{x^{1}=A\right\}$ as defined in Definition 1.8. We denote by $S_{g}(\lambda)$ and $S_{\tilde{g}}(\lambda)$ the corresponding scattering operators at a fixed energy $\lambda \neq 0$ as defined in Theorem 1.24. Assume that

$$
S_{g}(\lambda)=S_{\tilde{g}}(\lambda) .
$$

Then, there exists a diffeomorphism $\Psi: \mathcal{M} \rightarrow \mathcal{M}$, equals to the identity at the compactified ends $\left\{x^{1}=0\right\}$ and $\left\{x^{1}=A\right\}$, such that $\tilde{g}$ is the pull back of $g$ by $\Psi$, i.e.

$$
\tilde{g}=\Psi^{\star} g .
$$

For general Asymptotically Hyperbolic Manifolds (AHM in short) with no particular (hidden) symmetry, direct and inverse scattering results for scalar waves have been proved by Joshi and Sá Barreto in [42, by Sá Barreto in [65, by Guillarmou and Sá Barreto in [35, 36] and by Isozaki and Kurylev in [40]. In [42, it is shown that the asymptotics of the metric of an AHM are uniquely determined (up to isometries) by the scattering matrix $S_{g}(\lambda)$ at a fixed energy $\lambda$ off a discrete subset of $\mathbb{R}$. In [65, it is proved that the metric of an AHM is uniquely determined (up to isometries) by the scattering matrix $S_{g}(\lambda)$ for every $\lambda \in \mathbb{R}$ off an exceptional subset. Similar results are obtained recently in 40, for even more general classes of AHM. In [35, it is proved that, for connected conformally compact Einstein manifolds of even dimension $n+1$, the scattering matrix at energy $n$ on an open subset of its conformal boundary determines the manifold up to isometries. In [36, the authors study direct and inverse scattering problems for asymptotically complex hyperbolic manifolds and show that the topology and the metric of such a manifold are determined (up to invariants) by the scattering matrix at all energies. We also mention the work [56] of Marazzi in which the author study inverse scattering for the stationary Schrödinger equation with smooth potential not vanishing at the boundary on a conformally compact manifold with sectional curvature $-\alpha^{2}$ at the boundary. The author then shows that the scattering matrix at two fixed energies $\lambda_{1}$ and $\lambda_{2}, \lambda_{1} \neq \lambda_{2}$, in a suitable subset of $\mathbb{C}$, determines $\alpha$ and the Taylor series of both the potential and the metric at the boundary. At last, we also mention [14] where related inverse problems - inverse resonance problems - are studied in certain subclasses of AHM.

This work must also be put into perspective with the anisotropic Calderón problem on compact manifolds with boundary. We recall here, the definition of this problem. Let $(\mathcal{M}, g)$ be a riemannian compact manifold with smooth boundary $\partial \mathcal{M}$. We denote by $-\Delta_{g}$ the Laplace-Beltrami operator on 
$(\mathcal{M}, g)$ and we recall that this operator with Dirichlet boundary conditions is selfadjoint on $L^{2}\left(\mathcal{M}, d V\right.$ ol $\left._{g}\right)$ and has a pure point spectrum $\left\{\lambda_{j}^{2}\right\}_{j \geq 1}$. We are interested in the solutions $u$ of

$$
\left\{\begin{aligned}
-\Delta_{g} u & =\lambda^{2} u, \quad \text { on } \mathcal{M}, \\
u & =\psi, \quad \text { on } \partial \mathcal{M} .
\end{aligned}\right.
$$

It is known (see for instance [66]) that for any $\psi \in H^{\frac{1}{2}}(\partial \mathcal{M})$ there exits a unique weak solution $u \in H^{1}(\mathcal{M})$ of 1.18 when $\lambda^{2}$ does not belong to the Dirichlet spectrum $\left\{\lambda_{i}^{2}\right\}$ of $-\Delta_{g}$. This allows us to define the Dirichlet-to-Neumann $(\mathrm{DN})$ map as the operator $\Lambda_{g}\left(\lambda^{2}\right)$ from $H^{\frac{1}{2}}(\partial \mathcal{M})$ to $H^{-\frac{1}{2}}(\partial \mathcal{M})$ defined for all $\psi \in H^{\frac{1}{2}}(\partial \mathcal{M})$ by

$$
\Lambda_{g}\left(\lambda^{2}\right)(\psi)=\left(\partial_{\nu} u\right)_{\mid \partial \mathcal{M}},
$$

where $u$ is the unique solution of $(1.18)$ and $\left(\partial_{\nu} u\right)_{\mid \partial \mathcal{M}}$ is its normal derivative with respect to the unit outer normal vector $\nu$ on $\partial \mathcal{M}$. The anisotropic Calderón problem can be stated as:

Does the knowledge of the DN map $\Lambda_{g}\left(\lambda^{2}\right)$ at a frequency $\lambda^{2}$ determine uniquely the metric $g$ ?

We refer for instance to [27, 28, 36, 37, 47, 51, 52, 54, for important contributions to this subject and to the surveys [38, 48, 66, 71] for the current state of the art.

In dimension two, the anisotropic Calderón problem with $\lambda^{2}=0$ was shown to be true for smooth connected riemannian surfaces in [52, 54]. A positive answer for zero frequency $\lambda^{2}=0$ in dimension 3 or higher has been given for compact connected real analytic riemannian manifolds with real analytic boundary first in [54] under some topological assumptions relaxed later in [51, 52] and for compact connected Einstein manifolds with boundary in [35. The general anisotropic Calderón problem in dimension 3 or higher remains a major open problem. Results have been obtained recently in [27, 28] for some classes of smooth compact riemannian manifolds with boundary that are conformally transversally anisotropic, i.e. riemannian manifolds $(\mathcal{M}, g)$ such that

$$
\mathcal{M} \subset \subset \mathbb{R} \times \mathcal{M}_{0}, \quad g=c\left(e \oplus g_{0}\right),
$$

where $\left(\mathcal{M}_{0}, g_{0}\right)$ is a $n-1$ dimensional smooth compact riemannian manifold with boundary, $e$ is the euclidean metric on the real line, and $c$ is a smooth positive function on the cylinder $\mathbb{R} \times \mathcal{M}_{0}$. Under some conditions on the transverse manifold $\left(\mathcal{M}_{0}, g_{0}\right)$ (such as simplicity), the riemannian manifold $(\mathcal{M}, g)$ is said to be admissible. In that framework, the authors of [27, 28, were able to determine uniquely the conformal factor $c$ from the knowledge of the DN map at zero frequency $\lambda^{2}=0$. One of the aim of this paper is thus to give an example of manifolds on which we can solve the inverse scattering problem at fixed energy but do not have one of the particular structures we just described before for which the uniqueness for the anisotropic Calderón problem on compact manifolds with boundary is known (see Example $1.2,3)$ ).

\subsection{Overview of the proof}

The proof of Theorem 1.25 is divided into four steps which we describe here.

Step 1: The first step of the proof consists in solving the direct problem. This will be done in Section 2. In this Section we first use the structure of Stäckel manifold satisfying the Robertson condition to proceed to the separation of variables for the Helmholtz equation. We obtain that the shifted Helmholtz equation

$$
-\left(\Delta_{g}+1\right) f=\lambda^{2} f
$$


can be rewritten as

$$
A_{1} f+s_{12} L f+s_{13} H f=0,
$$

where $A_{1}$ is a differential operator in the variable $x^{1}$ alone and $L$ and $H$ are commuting, elliptic, semibounded selfadjoint operators on $L^{2}\left(\mathcal{T}^{2}, s^{11} d x^{2} d x^{3}\right)$ that only depend on the variables $x^{2}$ and $x^{3}$. Since the operators $L$ and $H$ commute, there exists a common Hilbertian basis of eigenfunctions for $H$ and $L$. Moreover, the ellipticity property on a compact manifold shows that the spectrum is discrete and the selfadjointness proves that the spectrum is real. We consider generalized harmonics $\left\{Y_{m}\right\}_{m \geq 1}$ which form a Hilbertian basis of $L^{2}\left(\mathcal{T}^{2}, s^{11} d x^{2} d x^{3}\right)$ associated with the coupled spectrum $\left(\mu_{m}^{2}, \nu_{m}^{2}\right)$ of $(H, L)$. We decompose the solutions $f=\sum_{m \geq 1} u_{m}\left(x^{1}\right) Y_{m}\left(x^{2}, x^{3}\right)$ of the Helmholtz equation on the common basis of harmonics $\left\{Y_{m}\right\}_{m \geq 1}$ and we then conclude that the Helmholtz equation separates into a system of three ordinary differential equations:

$$
\left\{\begin{array}{l}
-u_{m}^{\prime \prime}\left(x^{1}\right)+\frac{1}{2}\left(\log \left(f_{1}\right)\left(x^{1}\right)\right)^{\prime} u_{m}^{\prime}\left(x^{1}\right)+\left[-\left(\lambda^{2}+1\right) s_{11}\left(x^{1}\right)+\mu_{m}^{2} s_{12}\left(x^{1}\right)+\nu_{m}^{2} s_{13}\left(x^{1}\right)\right] u_{m}\left(x^{1}\right)=0 \\
-v_{m}^{\prime \prime}\left(x^{2}\right)+\left[-\left(\lambda^{2}+1\right) s_{21}\left(x^{2}\right)+\mu_{m}^{2} s_{22}\left(x^{2}\right)+\nu_{m}^{2} s_{23}\left(x^{2}\right)\right] v_{m}\left(x^{2}\right)=0 \\
-w_{m}^{\prime \prime}\left(x^{3}\right)+\left[-\left(\lambda^{2}+1\right) s_{31}\left(x^{3}\right)+\mu_{m}^{2} s_{32}\left(x^{3}\right)+\nu_{m}^{2} s_{33}\left(x^{3}\right)\right] w_{m}\left(x^{3}\right)=0
\end{array},\right.
$$

where $f_{1}$ is the function appearing in the Robertson condition and $Y_{m}\left(x^{2}, x^{3}\right)=v_{m}\left(x^{2}\right) w_{m}\left(x^{3}\right)$. In this system of ODEs there is one ODE in the radial variable $x^{1}$ and two ODEs in the angular variables $x^{2}$ and $x^{3}$. We emphasize that the angular momenta $\mu_{m}^{2}$ and $\nu_{m}^{2}$ which are the separation constants correspond also to the coupled spectrum of the two angular operators $H$ and $L$. The fact that the angular momenta $\left(\mu_{m}^{2}, \nu_{m}^{2}\right)$ are coupled has an important consequence in the use of the Complexification of the Angular Momentum method. Indeed, we cannot work separately with one angular momentum and we thus have to use a multivariable version of this method.

In a second time, we define the characteristic and Weyl-Titchmarsh functions following the construction given in $22,32,50$. We briefly recall here the definition of these objects and the reason why we use them. Using a Liouville change of variables $X^{1}=g\left(x^{1}\right), X^{1} \in\left(0, A^{1}\right)$ where $A^{1}=\int_{0}^{A} g\left(x^{1}\right) d x^{1}$, we can write the radial equation as

$$
-\ddot{U}+q_{\nu_{m}^{2}} U=-\mu_{m}^{2} U
$$

where $-\mu_{m}^{2}$ is now the spectral parameter and $q_{\nu_{m}^{2}}$ satisfies at the end $\left\{X^{1}=0\right\}$,

$$
q_{\nu_{m}^{2}}\left(X^{1}, \lambda\right)=-\frac{\lambda^{2}+\frac{1}{4}}{\left(X^{1}\right)^{2}}+q_{0, \nu_{m}^{2}}\left(X^{1}, \lambda\right),
$$

where $X^{1} q_{0, \nu_{m}^{2}}\left(X^{1}, \lambda\right)$ is integrable at the end $\left\{X^{1}=0\right\}$ (the potential $q_{\nu_{m}^{2}}$ also has the same property at the other end). We are thus in the framework of [32]. We can then define the characteristic and Weyl-Titchmarsh functions associated with this singular non-selfadjoint Schrödinger equation. To do this, we follow the method given in 22 . We thus define two fundamental systems of solutions $\left\{S_{10}, S_{20}\right\}$ and $\left\{S_{11}, S_{21}\right\}$ defined by

1. When $X^{1} \rightarrow 0$,

$$
S_{10}\left(X^{1}, \mu^{2}, \nu^{2}\right) \sim\left(X^{1}\right)^{\frac{1}{2}-i \lambda} \text { and } S_{20}\left(X^{1}, \mu^{2}, \nu^{2}\right) \sim \frac{1}{2 i \lambda}\left(X^{1}\right)^{\frac{1}{2}+i \lambda}
$$

and when $X^{1} \rightarrow A^{1}$

$$
S_{11}\left(X^{1}, \mu^{2}, \nu^{2}\right) \sim\left(A^{1}-X^{1}\right)^{\frac{1}{2}-i \lambda} \quad \text { and } \quad S_{21}\left(X^{1}, \mu^{2}, \nu^{2}\right) \sim-\frac{1}{2 i \lambda}\left(A^{1}-X^{1}\right)^{\frac{1}{2}+i \lambda} .
$$


2. $W\left(S_{1 n}, S_{2 n}\right)=1$ for $n \in\{0,1\}$.

3. For all $X^{1} \in\left(0, A^{1}\right), \mu \mapsto S_{j n}\left(X^{1}, \mu^{2}, \nu^{2}\right)$ is an entire function for $j \in\{1,2\}$ and $n \in\{0,1\}$.

We add some singular separated boundary conditions at the two ends (see (2.49) ) and we consider the new radial equation as an eigenvalue problem. Finally, we define the two characteristic functions of this radial equation as Wronskians of functions of the fundamental systems of solutions:

$$
\Delta_{q_{\nu_{m}^{2}}}\left(\mu_{m}^{2}\right)=W\left(S_{11}, S_{10}\right)
$$

and

$$
\delta_{q_{\nu_{m}^{2}}}\left(\mu_{m}^{2}\right)=W\left(S_{11}, S_{20}\right)
$$

and we also define the Weyl-Titchmarsh function by:

$$
M_{q_{\nu_{m}^{2}}}\left(\mu_{m}^{2}\right)=-\frac{\delta_{q_{\nu_{m}^{2}}}\left(\mu_{m}^{2}\right)}{\Delta_{q_{\nu_{m}^{2}}}\left(\mu_{m}^{2}\right)} .
$$

The above definition generalizes the usual definition of classical Weyl-Titchmarsh functions for regular Sturm-Liouville differential operators. We refer to [50] for the theory of selfadjoint singular SturmLiouville operators and the definition and main properties of Weyl-Titchmarsh functions. In our case the boundary conditions make the Sturm-Liouville equation non-selfadjoint. The generalized WeylTitchmarsh function can nevertheless be defined by the same recipe as shown in [22, 32] and recalled above. Our interest in considering the generalized Weyl-Titchmarsh function $M_{q_{\nu_{m}^{2}}}\left(\mu_{m}^{2}\right)$ comes from the fact that it is a powerful tool to prove uniqueness results for one-dimensional inverse problems. Indeed, roughly speaking, the Borg-Marchenko Theorem states (see [50]) that if $M_{q}$ and $M_{\tilde{q}}$ are two generalized Weyl-Titchmarsh functions associated with the equations

$$
-u^{\prime \prime}+q(x) u=-\mu^{2} u \quad \text { and } \quad-u^{\prime \prime}+\tilde{q}(x) u=-\mu^{2} u,
$$

where $q$ and $\tilde{q}$ satisfy the previous quadratic singularities at the ends, then if

$$
M_{q}\left(\mu^{2}\right)=M_{\tilde{q}}\left(\mu^{2}\right), \quad \mu \in \mathbb{C} \backslash\{\text { poles }\},
$$

then

$$
q=\tilde{q}
$$

We refer to [8, 33, 70] for results in the case of regular Weyl-Titchmarsh functions and to the recent results [32, 50] in the case of singular Weyl-Titchmarsh functions corresponding to possibly non-selfadjoint equation.

We note that, the characteristic and generalized Weyl-Titchmarsh functions obtained for each onedimensional equation $(1.19)$ can be summed up over the span of each of the harmonics $Y_{m}, m \geq 1$, in order to define operators from $L^{2}\left(\mathcal{T}^{2}, s^{11} d x^{2} d x^{3}\right)$ onto itself. Precisely, recalling that

$$
L^{2}\left(\mathcal{T}^{2}, s^{11} d x^{2} d x^{3}\right)=\bigoplus_{m \geq 1}\left\langle Y_{m}\right\rangle
$$

we define: 
Definition 1.11. Let $\lambda \neq 0$ be a fixed energy. The characteristic operator $\Delta(\lambda)$ and the generalized Weyl-Titchmarsh operator $M(\lambda)$ are defined as operators from $L^{2}\left(\mathcal{T}^{2}, s^{11} d x^{2} d x^{3}\right)$ onto itself that are diagonalizable on the Hilbert basis of eigenfunctions $\left\{Y_{m}\right\}_{m \geq 1}$ associated with the eigenvalues $\Delta_{q_{\nu_{m}^{2}}}\left(\mu_{m}^{2}\right)$ and $M_{q_{\nu_{m}^{2}}}\left(\mu_{m}^{2}\right)$. More precisely, for all $v \in L^{2}\left(\mathcal{T}^{2}, s^{11} d x^{2} d x^{3}\right), v$ can be decomposed as

$$
v=\sum_{m \geq 1} v_{m} Y_{m}, \quad v_{m} \in \mathbb{C}
$$

and we have

$$
\Delta(\lambda) v=\sum_{m \geq 1} \Delta_{q_{\nu_{m}^{2}}}\left(\mu_{m}^{2}\right) v_{m} Y_{m} \quad \text { and } \quad M(\lambda) v=\sum_{m \geq 1} M_{q_{\nu_{m}^{2}}}\left(\mu_{m}^{2}\right) v_{m} Y_{m}
$$

We emphasize that the separation of the variables allows us to "diagonalize" the reflection and the transmission operators into a countable family of multiplication operators by numbers $R_{g}\left(\lambda, \mu_{m}^{2}, \nu_{m}^{2}\right)$, $L_{g}\left(\lambda, \mu_{m}^{2}, \nu_{m}^{2}\right)$ and $T_{g}\left(\lambda, \mu_{m}^{2}, \nu_{m}^{2}\right)$ called reflection and transmission coefficients respectively. We will show (see Equations (2.59)-(2.61)) that the characteristic and Weyl-Titchmarsh functions are nothing but the transmission and the reflection coefficients respectively. The aim of this identification is to use the BorgMarchenko theorem from the equality of the scattering matrix at fixed energy.

Step 2: The second step of the proof consists in solving the inverse problem for the angular part of the Stäckel matrix. We begin our proof by a first reduction of our problem. Indeed, our main assumption is

$$
S_{g}(\lambda)=S_{\tilde{g}}(\lambda)
$$

and these operators act on $L^{2}\left(\mathcal{T}^{2}, s^{11} d x^{2} d x^{3}\right)$ and $L^{2}\left(\mathcal{T}^{2}, \tilde{s}^{11} d x^{2} d x^{3}\right)$ respectively. To compare these objects we thus must have

$$
s^{11}=\tilde{s}^{11} .
$$

Thanks to this equality and the gauge choice $f_{2}=f_{3}=1$, we will show easily that

$$
\left(\begin{array}{ll}
s_{22} & s_{23} \\
s_{32} & s_{33}
\end{array}\right)=\left(\begin{array}{ll}
\tilde{s}_{22} & \tilde{s}_{23} \\
\tilde{s}_{32} & \tilde{s}_{33}
\end{array}\right) G,
$$

where $G$ is a constant matrix of determinant 1 . As mentioned in the Introduction, the presence of the matrix $G$ is due to an invariance of the metric $g$ under a particular choice of the Stäckel matrix $S$. We can then assume that $G=I_{2}$ and we thus obtain

$$
\left(\begin{array}{ll}
s_{22} & s_{23} \\
s_{32} & s_{33}
\end{array}\right)=\left(\begin{array}{ll}
\tilde{s}_{22} & \tilde{s}_{23} \\
\tilde{s}_{32} & \tilde{s}_{33}
\end{array}\right) .
$$

Secondly, we want to show that $s_{21}=\tilde{s}_{21}$ and $s_{31}=\tilde{s}_{31}$. Using the particular structures of the operators $H$ and $L$, we can easily show that

$$
\left(\begin{array}{l}
\partial_{2}^{2} \\
\partial_{3}^{2}
\end{array}\right)=-\left(\begin{array}{ll}
s_{23} & s_{22} \\
s_{33} & s_{32}
\end{array}\right)\left(\begin{array}{c}
H \\
L
\end{array}\right)+\left(\lambda^{2}+1\right)\left(\begin{array}{l}
s_{21} \\
s_{31}
\end{array}\right) .
$$

We then apply Equation 1.24 on a vector of generalized harmonics

$$
\left(\begin{array}{c}
Y_{m} \\
Y_{m}
\end{array}\right)
$$


We use the decomposition onto the generalized harmonics to write $Y_{m}=\sum_{p \in E_{m}} c_{p} \tilde{Y}_{p}, m \geq 1$, on the Hilbertian basis of generalized harmonics $\left\{\tilde{Y}_{m}\right\}_{m \geq 1}$ and we identify for each $p \in E_{m}$ the coefficient of the harmonic $\tilde{Y}_{p}$. Hence, we obtain, thanks to 1.23 , that

$$
-\left(\begin{array}{ll}
s_{23} & s_{22} \\
s_{33} & s_{32}
\end{array}\right)\left(\begin{array}{c}
\mu_{m}^{2} \\
\nu_{m}^{2}
\end{array}\right)+\left(\lambda^{2}+1\right)\left(\begin{array}{l}
s_{21} \\
s_{31}
\end{array}\right)=-\left(\begin{array}{ll}
s_{23} & s_{22} \\
s_{33} & s_{32}
\end{array}\right)\left(\begin{array}{c}
\tilde{\mu}_{p}^{2} \\
\tilde{\nu}_{p}^{2}
\end{array}\right)+\left(\lambda^{2}+1\right)\left(\begin{array}{c}
\tilde{s}_{21} \\
\tilde{s}_{31}
\end{array}\right), \quad \forall p \in E_{m} .
$$

We put at the left-hand side the constants terms with respect to the variables $x^{2}$ and $x^{3}$ and the other terms at the right-hand side. We thus obtain that

$$
\left\{\begin{array}{l}
s_{21}\left(x^{2}\right)=\tilde{s}_{21}\left(x^{2}\right)-C_{1} s_{23}\left(x^{2}\right)-C_{2} s_{22}\left(x^{2}\right) \\
s_{31}\left(x^{3}\right)=\tilde{s}_{31}\left(x^{3}\right)-C_{1} s_{33}\left(x^{3}\right)-C_{2} s_{32}\left(x^{3}\right)
\end{array},\right.
$$

where $C_{1}$ and $C_{2}$ are real constants. We note that, as mentioned previously in the Introduction, these equalities describe an invariance of the metric $g$ under the definition of the Stäckel matrix $S$ and we can choose $C_{1}=C_{2}=0$. Finally, we obtain

$$
\left(\begin{array}{l}
s_{21} \\
s_{31}
\end{array}\right)=\left(\begin{array}{l}
\tilde{s}_{21} \\
\tilde{s}_{31}
\end{array}\right)
$$

We conclude Section 3 by noticing that, thanks to these results, $H=\tilde{H}$ and $L=\tilde{L}$. As a consequence, since the generalized harmonics only depend on $H$ and $L$, we can choose $Y_{m}=\tilde{Y}_{m}$ and

$$
\left(\begin{array}{c}
\mu_{m}^{2} \\
\nu_{m}^{2}
\end{array}\right)=\left(\begin{array}{c}
\tilde{\mu}_{m}^{2} \\
\tilde{\nu}_{m}^{2}
\end{array}\right), \quad \forall m \geq 1
$$

We emphasize that the choice of the generalized harmonics is not uniquely defined in each eigenspace associated with an eigenvalue with multiplicity higher than two. However, the scattering matrix does not depend on the choice of the $Y_{m}$ on each eigenspace.

Step 3: In a third step, we solve in Section 4 the inverse problem for the radial part of the Stäckel matrix. The main tool of this Section is a multivariable version of the Complex Angular Momentum method. The main assumption of Theorem 1.25 implies that,

$$
M\left(\mu_{m}^{2}, \nu_{m}^{2}\right)=\tilde{M}\left(\mu_{m}^{2}, \nu_{m}^{2}\right), \quad \forall m \geq 1 .
$$

Roughly speaking, the aim of the Complexification of the Angular Momentum method is the following: from a discrete set of informations (here the equality of the Weyl-Titchmarsh functions on the coupled spectrum) we want to obtain a continuous regime of informations (here the equality of these functions on $\mathbb{C}^{2}$ ). In other words, we want to extend the previous equality on $\mathbb{C}^{2}$, i.e. that we want to show that

$$
M\left(\mu^{2}, \nu^{2}\right)=\tilde{M}\left(\mu^{2}, \nu^{2}\right), \quad \forall(\mu, \nu) \in \mathbb{C}^{2} \backslash P,
$$

where $P$ is the set of points $(\mu, \nu) \in \mathbb{C}^{2}$ such that the Weyl-Titchmarsh functions does not exists, i.e. such that the denominator vanishes. We proceed as follows. Recalling the definition of the Weyl-Titchmarsh function given in 1.20 , we consider the application

$$
\begin{aligned}
\psi: \mathbb{C}^{2} & \rightarrow \mathbb{C} \\
(\mu, \nu) & \mapsto \tilde{\Delta}\left(\mu^{2}, \nu^{2}\right) \delta\left(\mu^{2}, \nu^{2}\right)-\Delta\left(\mu^{2}, \nu^{2}\right) \tilde{\delta}\left(\mu^{2}, \nu^{2}\right)
\end{aligned}
$$


and we want to show that $\psi$ is identically zero on $\mathbb{C}^{2}$. To obtain this fact we use an uniqueness result for multivariable holomorphic functions given in [10] which says roughly speaking that a holomorphic function which satisfies good estimates on a certain cone and which has enough zeros in this cone is identically zero. We thus first show that the function $\psi$ is holomorphic and of exponential type with respect to $\mu$ and $\nu$, i.e. that we can find three positive constants $A, B$ and $C$ such that $|\psi(\mu, \nu)|$ is less than $C \exp (A|\operatorname{Re}(\mu)|+B|\operatorname{Re}(\nu)|)$. Up to an exponential correction, we then obtain that $\psi$ is holomorphic and bounded on a certain cone of $\left(\mathbb{R}^{+}\right)^{2}$. Finally, we quantify the zeros of $\psi$ in this cone using the knowledge of the distribution of the coupled spectrum (on which the function $\psi$ vanishes) given in the works of Colin de Verdière [18, 19]. We can then conclude that $\psi=0$, i.e.

$$
M\left(\mu^{2}, \nu^{2}\right)=\tilde{M}\left(\mu^{2}, \nu^{2}\right), \quad \forall(\mu, \nu) \in \mathbb{C}^{2} \backslash P
$$

and, by definition, we deduce from this equality that,

$$
M_{q_{\nu^{2}}}\left(\mu^{2}\right)=M_{\tilde{q}_{\nu^{2}}}\left(\mu^{2}\right), \quad \forall(\mu, \nu) \in \mathbb{C}^{2} \backslash P .
$$

Step 4: We use the celebrated Borg-Marchenko Theorem (see [22, 32]) to obtain

$$
q_{\nu_{m}^{2}}=\tilde{q}_{\nu_{m}^{2}}, \quad \forall m \geq 1 .
$$

Since this equality is true for all $m \geq 1$, we can "decouple" the potential

$$
q_{\nu_{m}^{2}}=-\left(\lambda^{2}+1\right) \frac{s_{11}}{s_{12}}+\nu_{m}^{2} \frac{s_{13}}{s_{12}}+\frac{1}{16}\left(\left(\log \left(\frac{f_{1}}{s_{12}}\right)\right)\right)^{2}-\frac{1}{4}\left(\log \left(\frac{f_{1}}{s_{12}}\right)\right) .
$$

and we thus obtain the uniqueness of the quotient

$$
\frac{s_{13}}{s_{12}}
$$

and one ODE on the quotients

$$
\frac{f_{1}}{s_{12}}, \quad \frac{\tilde{f}_{1}}{\tilde{s}_{12}} \text { and } \frac{s_{11}}{s_{12}}, \quad \frac{\tilde{s}_{11}}{\tilde{s}_{12}} .
$$

We then rewrite this last ODE as a non-linear ODE on the function

$$
u=\left(\frac{s_{12}}{f_{1}} \frac{\tilde{f}_{1}}{\tilde{s}_{12}}\right)^{\frac{1}{4}}
$$

given by

$$
u^{\prime \prime}+\frac{1}{2}(\log (\tilde{h}))^{\prime} u^{\prime}+\left(\lambda^{2}+1\right) \tilde{h}\left(l s_{32}-s_{33}\right)\left(s_{23}-l s_{22}\right)\left(u^{5}-u\right)=0,
$$

where

$$
f=\frac{s_{11}}{s_{12}}, \quad h=\frac{s_{12}}{f_{1}} \quad \text { and } \quad l=\frac{s_{13}}{s_{12}}=\tilde{l} .
$$

Moreover, $u$ satisfies Cauchy conditions at the ends 0 and $A$ given by

$$
u(0)=u(A)=1 \quad \text { and } \quad u^{\prime}(0)=u^{\prime}(A)=0 .
$$

We note that $u=1$ is a solution of this system and by uniqueness of the Cauchy problem we conclude that $u=1$. We then have shown that

$$
\frac{f_{1}}{s_{12}}=\frac{\tilde{f}_{1}}{\tilde{s}_{12}}
$$


Finally, using the Robertson condition, we conclude that

$$
\frac{s_{11}}{s_{12}}=\frac{\tilde{s}_{11}}{\tilde{s}_{12}} \quad \text { and } \quad \frac{s_{11}}{s_{13}}=\frac{\tilde{s}_{11}}{\tilde{s}_{13}} .
$$

This finishes the proof of Step 4 and together with the previous steps, the proof of Theorem 1.25 . We emphasize that we transformed the implicit non-linear problem of determining the metric from the knowledge of the scattering matrix at fixed energy into an explicit non-linear problem consisting in solving the Cauchy problem associated with the non-linear ODE (1.25).

This paper is organized as follows. In Section 2 we solve the direct problem. In this Section we study the separation of variables for the Helmholtz equation, we define the characteristic and WeylTitchmarsh functions for different choices of spectral parameters and we make the link between these different functions and the scattering coefficients. In Section 3 we solve the inverse problem for the angular part of the Stäckel matrix. In Section 4 we solve the inverse problem for the radial part of the Stäckel matrix using a multivariable version of the Complex Angular Momentum method. Finally, in Section 5 , we finish the proof of our main Theorem 1.25 .

\section{The direct problem}

In this Section we will study the direct scattering problem for the shifted Helmholtz equation 2.26). We first study the separation of the Helmholtz equation. Secondly, we define several characteristic and generalized Weyl-Titchmarsh functions associated with unidimensional Schrödinger equations in the radial variable corresponding to different choices of spectral parameters and we study the link between these functions and the scattering operator associated with the Helmholtz equation.

\subsection{Separation of variables for the Helmholtz equation}

We consider (see [13, 40, 41, 42]) the shifted stationary Helmholtz equation

$$
-\left(\Delta_{g}+1\right) f=\lambda^{2} f
$$

where $\lambda \neq 0$ is a fixed energy, which is usually studied in case of asymptotically hyperbolic manifolds (see [13, 40, 41, 42]). Indeed, it is known (see [41]) that the essential spectrum of $-\Delta_{g}$ is $[1,+\infty$ ) and thus, we shift the bottom of the essential spectrum to 0 . It is known that the operator $-\Delta_{g}-1$ has no eigenvalues embedded into the essential spectrum $[0,+\infty)$ (see [15, 40, 41]). We know that there exists a coordinates system separable for the Helmholtz equation 2.26) if and only if the metric (1.4) is in Stäckel form and furthermore if the Robertson condition (1.6) is satisfied. We emphasize that, contrary to the case $n=2$ studied in [22, we really need the Robertson condition in the case $n=3$.

Lemma 2.1. The Helmholtz equation 2.26) can be rewritten as

$$
A_{1} f+s_{12} L f+s_{13} H f=0,
$$

where,

$$
A_{i}=-\partial_{i}^{2}+\frac{1}{2} \partial_{i}\left(\log \left(f_{i}\right)\right) \partial_{i}-\left(\lambda^{2}+1\right) s_{i 1}, \quad \text { for } \quad i \in\{1,2,3\}
$$

and

$$
L=-\frac{s_{33}}{s^{11}} A_{2}+\frac{s_{23}}{s^{11}} A_{3} \quad \text { and } \quad H=\frac{s_{32}}{s^{11}} A_{2}-\frac{s_{22}}{s^{11}} A_{3} .
$$


Proof. We recall that the Laplace-Beltrami operator is given in the global coordinates system $\left(x^{i}\right)_{i=1,2,3}$ by

$$
\Delta_{g}=\frac{1}{\sqrt{|g|}} \partial_{i}\left(\sqrt{|g|} g^{i j} \partial_{j}\right)
$$

where $|g|$ is the determinant of the metric and $\left(g^{i j}\right)$ is the inverse of the metric $\left(g_{i j}\right)$. Using the fact that

$$
g^{i i}=\frac{1}{H_{i}^{2}}, \quad \sqrt{|g|}=H_{1} H_{2} H_{3},
$$

and the Robertson condition 1.7 we easily show that

$$
\Delta_{g}=\sum_{i=1}^{3} \frac{1}{H_{i}^{2}}\left(\partial_{i}^{2}-\frac{1}{2} \partial_{i}\left(\log \left(f_{i}\right)\right) \partial_{i}\right) .
$$

Remark 2.2. We note that the Robertson condition is equivalent to the existence of three functions $f_{i}=f_{i}\left(x^{i}\right)$ such that

$$
\partial_{i} \log \left(\frac{H_{i}^{4}}{H_{1}^{2} H_{2}^{2} H_{3}^{2}}\right)=\partial_{i} \log \left(f_{i}\right), \quad \forall i \in\{1,2,3\} .
$$

This equality is interesting since it gives us an expression of the Robertson condition directly in terms of the coefficients $H_{i}^{2}$ of the metric $g$.

Hence, from 2.30 we immediately obtain that the Helmholtz equation 2.26 can be written as

$$
\sum_{i=1}^{3} \frac{1}{H_{i}^{2}} A_{i}^{0} f=\left(\lambda^{2}+1\right) f
$$

where

$$
A_{i}^{0}=-\partial_{i}^{2}+\frac{1}{2} \partial_{i}\left(\log \left(f_{i}\right)\right) \partial_{i}, \quad \text { for } \quad i \in\{1,2,3\} .
$$

If we multiply Equation 2.31 by $H_{1}^{2}$ and if we use that

$$
H_{1}^{2}=s_{11}+s_{21} \frac{s^{21}}{s^{11}}+s_{31} \frac{s^{31}}{s^{11}}, \quad \frac{H_{1}^{2}}{H_{2}^{2}}=\frac{s^{21}}{s^{11}} \quad \text { and } \quad \frac{H_{1}^{2}}{H_{3}^{2}}=\frac{s^{31}}{s^{11}},
$$

we obtain

$$
A_{1} f+\frac{s^{21}}{s^{11}} A_{2} f+\frac{s^{31}}{s^{11}} A_{3} f=0 .
$$

Finally, using the equalities

$$
\frac{s^{21}}{s^{11}}=-s_{12} \frac{s_{33}}{s^{11}}+s_{13} \frac{s_{32}}{s^{11}} \quad \text { and } \quad \frac{s^{31}}{s^{11}}=s_{12} \frac{s_{23}}{s^{11}}-s_{13} \frac{s_{22}}{s^{11}},
$$

we obtain from 2.33 the equation

$$
A_{1} f+s_{12} L f+s_{13} H f=0,
$$

where the operators $H$ and $L$ are given by 2.29 . 
Remark 2.3. Since we assumed that $f_{2}$ and $f_{3}$ are constant functions equal to 1 (see Remark 1.22) we know that

$$
A_{2}^{0}=-\partial_{2}^{2} \quad \text { and } \quad A_{3}^{0}=-\partial_{3}^{2} .
$$

Remark 2.4. We can make the link between the angular operators $H$ and $L$ and the operators $\hat{P}_{2}$ and $\hat{P}_{3}$ related to the existence of Killing 2-tensors as introduced in Theorem 1.14. To do this we follow the construction given in [45]. We thus consider, according to Equation (2.21) of [45], for $i \in\{1,2,3\}$, the operators

$$
\hat{P}_{i}=\sum_{j=1}^{3} \frac{s^{j i}}{\operatorname{det}(S)}\left(\partial_{j}^{2}-\frac{1}{2} \partial_{j} \log \left(f_{j}\right) \partial_{j}\right)
$$

In other words,

$$
\left(\begin{array}{l}
\hat{P}_{1} \\
\hat{P}_{2} \\
\hat{P}_{3}
\end{array}\right)=-S^{-1}\left(\begin{array}{l}
A_{1}^{0} \\
A_{2}^{0} \\
A_{3}^{0}
\end{array}\right)
$$

where $A_{i}^{0}$ were defined in 2.32). We note that

$$
\hat{P}_{1}=\sum_{j=1}^{3} \frac{s^{j 1}}{\operatorname{det}(S)}\left(\partial_{j}^{2}-\frac{1}{2} \partial_{j} \log \left(f_{j}\right) \partial_{j}\right)=\sum_{j=1}^{3} \frac{1}{H_{j}^{2}}\left(\partial_{j}^{2}-\frac{1}{2} \partial_{j} \log \left(f_{j}\right) \partial_{j}\right)=\Delta_{g} .
$$

Since,

we see that

$$
\left(\begin{array}{c}
A_{1}^{0} \\
A_{2}^{0} \\
A_{3}^{0}
\end{array}\right)=-S\left(\begin{array}{c}
\hat{P}_{1} \\
\hat{P}_{2} \\
\hat{P}_{3}
\end{array}\right)
$$

$$
\left(\begin{array}{l}
A_{2}^{0} \\
A_{3}^{0}
\end{array}\right)=-\left(\begin{array}{ll}
s_{22} & s_{23} \\
s_{32} & s_{33}
\end{array}\right)\left(\begin{array}{l}
\hat{P}_{2} \\
\hat{P}_{3}
\end{array}\right)-\left(\begin{array}{c}
s_{21} \hat{P}_{1} \\
s_{31} \hat{P}_{1}
\end{array}\right) .
$$

Applied to solutions of the Helmholtz equation (2.26) these operators coincide with

$$
\left(\begin{array}{l}
A_{2}^{0}-\left(\lambda^{2}+1\right) s_{21} \\
A_{3}^{0}-\left(\lambda^{2}+1\right) s_{31}
\end{array}\right)=-\left(\begin{array}{ll}
s_{22} & s_{23} \\
s_{32} & s_{33}
\end{array}\right)\left(\begin{array}{l}
\hat{P}_{2} \\
\hat{P}_{3}
\end{array}\right) .
$$

Since

it follows that

$$
\left(\begin{array}{l}
A_{2}^{0}-\left(\lambda^{2}+1\right) s_{21} \\
A_{3}^{0}-\left(\lambda^{2}+1\right) s_{31}
\end{array}\right)=\left(\begin{array}{l}
A_{2} \\
A_{3}
\end{array}\right)
$$

$$
\left(\begin{array}{l}
\hat{P}_{2} \\
\hat{P}_{3}
\end{array}\right)=-\frac{1}{s^{11}}\left(\begin{array}{cc}
s_{33} & -s_{23} \\
-s_{32} & s_{22}
\end{array}\right)\left(\begin{array}{l}
A_{2} \\
A_{3}
\end{array}\right) .
$$

In other words, when applied to solution of (2.26), we have

or

$$
\hat{P}_{2}=-\frac{s_{33}}{s^{11}} A_{2}+\frac{s_{23}}{s^{11}} A_{3} \quad \text { and } \quad \hat{P}_{3}=\frac{s_{32}}{s^{11}} A_{2}-\frac{s_{22}}{s^{11}} A_{3},
$$

$$
\hat{P}_{2}=L \quad \text { and } \quad \hat{P}_{3}=H .
$$

We emphasize that the operators $L$ and $H$ and the operators $\hat{P}_{2}$ and $\hat{P}_{3}$ respectively coincide only if we apply them to solutions of the Helmholtz equation [2.26]. Moreover, thanks to [45] we know that $\left[\hat{P}_{2}, \hat{P}_{3}\right]=0$. Thus, $L$ and $H$ are commuting operators. Moreover, the coupled eigenvalues of the operators $L$ and $H$ correspond to the separation constants of the Helmholtz equation. 
The operators $L$ and $H$ are of great interest in our study. In particular we will show that $L$ and $H$ are elliptic operators in the sense of the definition of ellipticity given in [46] which we recall here. Let $a(y, D)$ be a differential operator given in local coordinates by

$$
(a(y, D) f)(y)=-a^{j k}(y) \partial_{j} \partial_{k} f(y)-b^{j}(y) \partial_{j} f(y)-c(y) f(y),
$$

where $y=\left(x^{2}, x^{3}\right), \partial_{j}=\frac{\partial}{\partial x^{j}}, j \in\{2,3\}$, the coefficients are real and $\left(a^{j k}\right)$ is a symmetric matrix. The differential operator $a(y, D)$ is then said to be elliptic if the matrix $\left(a^{j k}\right)$ is positive definite. We can first prove the following lemma.

Lemma 2.5. The operators $L$ and $H$ satisfy the following properties:

1. $L H=H L$.

2. $L$ and $H$ are elliptic operators.

3. $L$ and $H$ are selfadjoint operators on the space $L^{2}\left(\mathcal{T}^{2}, s^{11} d x^{2} d x^{3}\right)$.

4. $L$ and $H$ are semibounded operators.

Proof. 1. The proof of the commutativity of the operators $L$ and $H$ is quite easy since $A_{2}$ and $A_{3}$ are commuting operators and $s_{22}$ and $s_{23}$ only depend on $x^{2}$ whereas $s_{32}$ and $s_{33}$ only depend on $x^{3}$. We note that, thanks to the fact that $\hat{P}_{2}=L$ and $\hat{P}_{3}=H$, we already know that these operators commute thanks to [45].

2. Using the definitions of $L$ and $H$ given in 2.29), we obtain that $L$ is an elliptic operator if and only if the matrix

$$
\left(\begin{array}{cc}
-\frac{s_{33}}{s^{11}} & 0 \\
0 & \frac{s_{23}}{s^{11}}
\end{array}\right)
$$

is positive-definite whereas $H$ is an elliptic operator if and only if the matrix

$$
\left(\begin{array}{cc}
\frac{s_{32}}{s^{11}} & 0 \\
0 & -\frac{s_{22}}{s^{11}}
\end{array}\right)
$$

is positive-definite. We now recall that $s_{22}, s_{33}<0$ and $s_{23}, s_{32}>0$ (see condition (C) in Proposition 1.17) and that $s^{11}>0$ (see Remark (1.18)). We can thus conclude that $L$ and $H$ are elliptic operators.

3. We just study the operator $L$ since the proof is similar for the operator $H$. We first note that, to find the weight $s^{11}$ we can use the exercise 2.19 of [46] which says that an operator $A$ defined by

$$
(A f)(y)=(a(y, D) f)(y)=-a^{j k}(y) \partial_{j} \partial_{k} f(y)+b^{j}(y) \partial_{j} f(y)+c(y) f(y),
$$

is selfadjoint on $L^{2}\left(\mathcal{T}_{y}^{2}, m g^{\frac{1}{2}} d y\right)$ if and only if

$$
a(y, D) f=-\frac{1}{m g^{\frac{1}{2}}} \partial_{i}\left(m g^{\frac{1}{2}} a^{i j} \partial_{j} f\right)+q f .
$$

We recall that

$$
L=\frac{s_{33}}{s^{11}} \partial_{2}^{2}-\frac{s_{23}}{s^{11}} \partial_{3}^{2}+q\left(x^{2}, x^{3}\right) .
$$


Thus,

$$
\langle L u, v\rangle=\left\langle\frac{s_{33}}{s^{11}} \partial_{2}^{2} u, v\right\rangle+\left\langle-\frac{s_{23}}{s^{11}} \partial_{3}^{2} u, v\right\rangle+\langle q u, v\rangle
$$

Moreover,

$$
\left\langle\frac{s_{33}}{s^{11}} \partial_{2}^{2} u, v\right\rangle=\int_{\mathcal{T}^{2}} s_{33}\left(\partial_{2}^{2} u\right) v d x^{2} d x^{3}=\int_{\mathcal{T}^{2}} s_{33} u\left(\partial_{2}^{2} v\right) d x^{2} d x^{3},
$$

since the boundary terms vanish by periodicity and the function $s_{33}$ does not depend on $x^{2}$. Thus,

$$
\left\langle\frac{s_{33}}{s^{11}} \partial_{2}^{2} u, v\right\rangle=\left\langle u, \frac{s_{33}}{s^{11}} \partial_{2}^{2} v\right\rangle \text {. }
$$

The second and the third terms can be treat following the same procedure. Finally, we have shown that $L$ is selfadjoint on the space $L^{2}\left(\mathcal{T}^{2}, s^{11} d x^{2} d x^{3}\right)$.

4. Since the proof is similar for the operator $H$ we just give the proof for the operator $L$.

$$
\begin{aligned}
\langle L u, u\rangle & =\left\langle\left(-\frac{s_{33}}{s^{11}} A_{2}+\frac{s_{23}}{s^{11}} A_{3}\right) u, u\right\rangle \\
& =\left\langle\frac{s_{33}}{s^{11}} \partial_{2}^{2} u, u\right\rangle+\left\langle-\frac{s_{23}}{s^{11}} \partial_{3}^{2} u, u\right\rangle+\left(\lambda^{2}+1\right)\left\langle\frac{s_{33} s_{21}-s_{23} s_{31}}{s^{11}} u, u\right\rangle .
\end{aligned}
$$

We now study each of these terms.

$$
\begin{aligned}
\left\langle\frac{s_{33}}{s^{11}} \partial_{2}^{2} u, u\right\rangle & =\int_{\mathcal{T}^{2}} \frac{s_{33}}{s^{11}}\left(\partial_{2}^{2} u\right) u s^{11} d x^{2} d x^{3} \\
& =\int_{\mathcal{T}^{2}} s_{33}\left(\partial_{2}^{2} u\right) u d x^{2} d x^{3} \\
& =\underbrace{\left[s_{33}\left(\partial_{2} u\right) u\right]}_{=0 \text { by periodicity }}+\int_{\mathcal{T}^{2}}\left(-s_{33}\right)\left(\partial_{2} u\right)^{2} d x^{2} d x^{3} \\
& \geq 0,
\end{aligned}
$$

since $s_{33}<0$. Similarly,

$$
\left\langle-\frac{s_{23}}{s^{11}} \partial_{3}^{2} u, u\right\rangle \geq 0
$$

At last, since $s_{i j} \in C^{\infty}\left(\mathcal{T}^{2}\right)$ for $i \in\{2,3\}$ and $j \in\{1,2,3\}$, there exists $m \in \mathbb{R}$ such that

$$
\left(\lambda^{2}+1\right)\left\langle\frac{s_{33} s_{21}-s_{23} s_{31}}{s^{11}} u, u\right\rangle \geq m\langle u, u\rangle .
$$

Remark 2.6. Since the operators $L$ and $H$ commute, there exists a common Hilbertian basis of eigenfunctions of $H$ and L. Moreover, the ellipticity property on a compact manifold shows that the spectrum is discrete and the selfadjointness proves that the spectrum is real. The generalized harmonics $\left\{Y_{m}\right\}_{m \geq 1}$, associated with the coupled or joint spectrum $\left(\mu_{m}^{2}, \nu_{m}^{2}\right)$ for $(H, L)$, form a Hilbertian basis of $L^{2}\left(\mathcal{T}^{2}, s^{11} d x^{2} d x^{3}\right)$, i.e.

$$
H Y_{m}=\mu_{m}^{2} Y_{m} \quad \text { and } \quad L Y_{m}=\nu_{m}^{2} Y_{m}, \quad \forall m \geq 1,
$$

and

$$
L^{2}\left(\mathcal{T}^{2}, s^{11} d x^{2} d x^{3}\right)=\bigoplus_{m \geq 1}\left\langle Y_{m}\right\rangle
$$

Here, we order the coupled spectrum $\left(\mu_{m}^{2}, \nu_{m}^{2}\right)$ such that 
1. Counting multiplicity:

$$
\mu_{1}^{2}<\mu_{2}^{2} \leq \mu_{3}^{2} \leq \mu_{4}^{2} \leq \ldots \leq \mu_{n}^{2} \leq \ldots \rightarrow \infty .
$$

2. Starting from $n=1$ and by induction on $n$, for each $n \geq 1$ such that $\mu_{n}^{2}$ has multiplicity $k$, i.e. $\mu_{n}^{2}=\mu_{n+1}^{2}=\ldots=\mu_{n+k-1}^{2}$, we order the corresponding $\left(\nu_{j}^{2}\right)_{n \leq j \leq n+k-1}$ in increasing order, i.e., counting multiplicity,

$$
\nu_{n}^{2} \leq \nu_{n+1}^{2} \leq \ldots \leq \nu_{n+k-1}^{2} .
$$

The toric cylinder's topology implies that the boundary conditions are compatible with the decomposition on the common harmonics $\left\{Y_{m}\right\}_{m \geq 1}$ of $H$ and $L$. We thus look for solutions of 2.26) under the form

$$
f\left(x^{1}, x^{2}, x^{3}\right)=\sum_{m \geq 1} u_{m}\left(x^{1}\right) Y_{m}\left(x^{2}, x^{3}\right) .
$$

We use 2.35) in 2.27) and we obtain that $u_{m}$ satisfies, for all $m \geq 1$,

$$
-u^{\prime \prime}\left(x^{1}\right)+\frac{1}{2}\left(\log \left(f_{1}\right)\left(x^{1}\right)\right)^{\prime} u^{\prime}\left(x^{1}\right)+\left[-\left(\lambda^{2}+1\right) s_{11}\left(x^{1}\right)+\mu_{m}^{2} s_{12}\left(x^{1}\right)+\nu_{m}^{2} s_{13}\left(x^{1}\right)\right] u\left(x^{1}\right)=0 .
$$

Finally, inverting (2.34), we obtain

$$
\left\{\begin{array}{l}
A_{2} Y_{m}=-\left(s_{22} \mu_{m}^{2}+s_{23} \nu_{m}^{2}\right) Y_{m} \\
A_{3} Y_{m}=-\left(s_{32} \mu_{m}^{2}+s_{33} \nu_{m}^{2}\right) Y_{m}
\end{array}\right.
$$

Remark 2.7. The harmonics $Y_{m}\left(x^{2}, x^{3}\right), m \geq 1$, can be written as a product of a function of the variable $x^{2}$ and a function of the variable $x^{3}$. Let $\left(f_{2}, g_{2}\right)$ and $\left(f_{3}, g_{3}\right)$ be periodic fundamental systems of solutions associated with the operators $A_{2}$ and $A_{3}$ respectively. We can thus write $Y_{m}\left(x^{2}, x^{3}\right)$ as

$$
Y_{m}\left(x^{2}, x^{3}\right)=a\left(x^{3}\right) f_{2}\left(x^{2}\right)+b\left(x^{3}\right) g_{2}\left(x^{2}\right) .
$$

We then apply the operator $A_{3}$ on this equality and we obtain that

$$
A_{3}\left(Y_{m}\right)\left(x^{2}, x^{3}\right)=A_{3}(a)\left(x^{3}\right) f_{2}\left(x^{2}\right)+A_{3}(b)\left(x^{3}\right) g_{2}\left(x^{2}\right) .
$$

Thus, using that $A_{3} Y_{m}=-\left(s_{32} \mu_{m}^{2}+s_{33} \nu_{m}^{2}\right) Y_{m}$ and the fact that $\left(f_{2}, g_{2}\right)$ is a fundamental system of solutions we obtain that

$$
Y_{m}\left(x^{2}, x^{3}\right)=a f_{2}\left(x^{2}\right) f_{3}\left(x^{3}\right)+b f_{2}\left(x^{2}\right) g_{3}\left(x^{3}\right)+c g_{2}\left(x^{2}\right) f_{3}\left(x^{3}\right)+d g_{2}\left(x^{2}\right) g_{3}\left(x^{3}\right),
$$

where $a, b, c$ and $d$ are real constants. Thus, for each coupled eigenvalue $\left(\mu_{m}^{2}, \nu_{m}^{2}\right), m \geq 1$, the corresponding eigenspace for the couple of operator $(H, L)$ is at most of dimension four. However, the diagonalization of the scattering matrix $S_{g}(\lambda)$ does not depend on the choice of the harmonics in each eigenspace associated with a coupled eigenvalue $\left(\mu_{m}^{2}, \nu_{m}^{2}\right)$ and we can thus choose as harmonics: $Y_{m}=f_{2} f_{3}, Y_{m}=f_{2} g_{3}$, $Y_{m}=g_{2} f_{3}$ and $Y_{m}=g_{2} g_{3}$. We can then assume that $Y_{m}\left(x^{2}, x^{3}\right)$ is a product of a function of the variable $x^{2}$ and a function of the variable $x^{3}$.

Lemma 2.8. Any solution $u \in H^{1}(\mathcal{M})$ of $-\left(\Delta_{g}+1\right) u=\lambda^{2} u$, can be written as

$$
u=\sum_{m \geq 1} u_{m}\left(x^{1}\right) Y_{m}\left(x^{2}, x^{3}\right),
$$


where $Y_{m}\left(x^{2}, x^{3}\right)=v_{m}\left(x^{2}\right) w_{m}\left(x^{3}\right)$ and

$$
\left\{\begin{array}{l}
-u_{m}^{\prime \prime}\left(x^{1}\right)+\frac{1}{2}\left(\log \left(f_{1}\right)\left(x^{1}\right)\right)^{\prime} u_{m}^{\prime}\left(x^{1}\right)+\left[-\left(\lambda^{2}+1\right) s_{11}\left(x^{1}\right)+\mu_{m}^{2} s_{12}\left(x^{1}\right)+\nu_{m}^{2} s_{13}\left(x^{1}\right)\right] u_{m}\left(x^{1}\right)=0 \\
-v_{m}^{\prime \prime}\left(x^{2}\right)+\left[-\left(\lambda^{2}+1\right) s_{21}\left(x^{2}\right)+\mu_{m}^{2} s_{22}\left(x^{2}\right)+\nu_{m}^{2} s_{23}\left(x^{2}\right)\right] v_{m}\left(x^{2}\right)=0 \\
-w_{m}^{\prime \prime}\left(x^{3}\right)+\left[-\left(\lambda^{2}+1\right) s_{31}\left(x^{3}\right)+\mu_{m}^{2} s_{32}\left(x^{3}\right)+\nu_{m}^{2} s_{33}\left(x^{3}\right)\right] w_{m}\left(x^{3}\right)=0
\end{array} .\right.
$$

From Lemma 2.8 we can deduce more informations on the eigenvalues $\left(\mu_{m}^{2}\right)_{m \geq 1}$ and $\left(\nu_{m}^{2}\right)_{m \geq 1}$. Indeed, we can prove the following Lemma which will be useful in the following.

Lemma 2.9. There exist real constants $C_{1}, C_{2}, D_{1}$ and $D_{2}$ such that for all $m \geq 1$,

$$
C_{1} \mu_{m}^{2}+D_{1} \leq \nu_{m}^{2} \leq C_{2} \mu_{m}^{2}+D_{2}
$$

where

$$
C_{1}=\min \left(-\frac{s_{32}}{s_{33}}\right)>0 \quad \text { and } \quad C_{2}=-\min \left(\frac{s_{22}}{s_{23}}\right)>0 .
$$

Proof. We first recall the angular equations of Lemma 2.8 .

$$
-v^{\prime \prime}\left(x^{2}\right)+\left[-\left(\lambda^{2}+1\right) s_{21}\left(x^{2}\right)+\mu_{m}^{2} s_{22}\left(x^{2}\right)+\nu_{m}^{2} s_{23}\left(x^{2}\right)\right] v\left(x^{2}\right)=0
$$

and

$$
-w^{\prime \prime}\left(x^{3}\right)+\left[-\left(\lambda^{2}+1\right) s_{31}\left(x^{3}\right)+\mu_{m}^{2} s_{32}\left(x^{3}\right)+\nu_{m}^{2} s_{33}\left(x^{3}\right)\right] w\left(x^{3}\right)=0 .
$$

We use a Liouville change of variables in 2.37) to transform this equation into a Schrödinger equation in which $-\nu_{m}^{2}$ is the spectral parameter. Thus, we define the diffeomorphism

$$
X^{2}=g_{2}\left(x^{2}\right)=\int_{0}^{x^{2}} \sqrt{s_{23}(t)} \mathrm{d} t
$$

and we define

$$
v\left(X^{2}, \mu_{m}^{2}, \nu_{m}^{2}\right)=v\left(h_{2}\left(X^{2}\right), \mu_{m}^{2}, \nu_{m}^{2}\right)
$$

where $h_{2}=g_{2}^{-1}$ is the inverse function of $g_{2}$. We also introduce a weight function to cancel the first order term. We thus define

$$
V\left(X^{2}, \mu_{m}^{2}, \nu_{m}^{2}\right)=\left(\frac{1}{s_{23}\left(h_{2}\left(X^{2}\right)\right)}\right)^{-\frac{1}{4}} v\left(h_{2}\left(X^{2}\right), \mu_{m}^{2}, \nu_{m}^{2}\right) .
$$

After calculation, we obtain that $V\left(X^{2}, \mu_{m}^{2}, \nu_{m}^{2}\right)$ satisfies, in the variable $X^{2}$, the Schrödinger equation

$$
-\ddot{V}\left(X^{2}, \mu_{m}^{2}, \nu_{m}^{2}\right)+p_{\mu_{m}^{2}, 2}\left(X^{2}, \lambda\right) V\left(X^{2}, \mu_{m}^{2}, \nu_{m}^{2}\right)=-\nu_{m}^{2} V\left(X^{2}, \mu_{m}^{2}, \nu_{m}^{2}\right)
$$

where,

$$
p_{\mu_{m}^{2}, 2}\left(X^{2}, \lambda\right)=-\left(\lambda^{2}+1\right) \frac{s_{21}\left(X^{2}\right)}{s_{23}\left(X^{2}\right)}+\mu_{m}^{2} \frac{s_{22}\left(X^{2}\right)}{s_{23}\left(X^{2}\right)},
$$

with $s_{21}\left(X^{2}\right):=s_{21}\left(h_{2}\left(X^{2}\right)\right), s_{22}\left(X^{2}\right):=s_{22}\left(h_{2}\left(X^{2}\right)\right)$ and $s_{23}\left(X^{2}\right):=s_{23}\left(h_{2}\left(X^{2}\right)\right)$. We follow the same procedure for 2.38 putting

$$
X^{3}=g_{3}\left(x^{3}\right)=\int_{0}^{x^{3}} \sqrt{-s_{33}(t)} \mathrm{d} t \quad \text { and } \quad W\left(X^{3}, \mu_{m}^{2}, \nu_{m}^{2}\right)=\left(\frac{1}{-s_{33}\left(h_{3}\left(X^{3}\right)\right)}\right)^{-\frac{1}{4}} w\left(h_{3}\left(X^{3}\right), \mu_{m}^{2}, \nu_{m}^{2}\right)
$$


and we obtain that $W\left(X^{3}\right)$ satisfies, in the variable $X^{3}$, the Schrödinger equation

$$
-\ddot{W}\left(X^{3}, \mu_{m}^{2}, \nu_{m}^{2}\right)+p_{\mu_{m}^{2}, 3}\left(X^{3}, \lambda\right) W\left(X^{3}, \mu_{m}^{2}, \nu_{m}^{2}\right)=\nu_{m}^{2} W\left(X^{3}, \mu_{m}^{2}, \nu_{m}^{2}\right),
$$

where,

$$
p_{\mu_{m}^{2}, 3}\left(X^{3}, \lambda\right)=\left(\lambda^{2}+1\right) \frac{s_{31}\left(X^{3}\right)}{s_{33}\left(X^{3}\right)}-\mu_{m}^{2} \frac{s_{32}\left(X^{3}\right)}{s_{33}\left(X^{3}\right)},
$$

with $s_{31}\left(X^{3}\right):=s_{31}\left(h_{3}\left(X^{3}\right)\right), s_{32}\left(X^{3}\right):=s_{32}\left(h_{3}\left(X^{3}\right)\right)$ and $s_{33}\left(X^{3}\right):=s_{33}\left(h_{3}\left(X^{3}\right)\right)$. Assume now that $\mu_{m}^{2}$ is fixed and look at 2.39) and 2.41) as eigenvalue problems in $\pm \nu_{m}^{2}$. We suppose that $\mu_{m}^{2}$ has multiplicity $k \geq 1$ and we use the notations given in Remark 2.6 , i.e. that we want to show that

$$
C_{1} \mu_{m}^{2}+D_{2} \leq \nu_{j}^{2} \leq C_{2} \mu_{m}^{2}+D_{2}, \quad \forall m \leq j \leq m+k-1,
$$

where $\nu_{j}^{2} \leq \nu_{j+1}^{2}$ for all $j \in\{m, \ldots, m+k-1\}$. We know that the spectra of the operators

$$
P_{2}=-\frac{\mathrm{d}^{2}}{\left(\mathrm{~d} X^{2}\right)^{2}}+p_{\mu_{m}^{2}, 2} \quad \text { and } \quad P_{3}=-\frac{\mathrm{d}^{2}}{\left(\mathrm{~d} X^{3}\right)^{2}}+p_{\mu_{m}^{2}, 3}
$$

are included in

$$
\left[\min \left(p_{\mu_{m}^{2}, 2}\right),+\infty\right) \text { and }\left[\min \left(p_{\mu_{m}^{2}, 3}\right),+\infty\right),
$$

respectively. The first condition gives us that

$$
-\nu_{j}^{2} \geq-C_{2} \mu_{m}^{2}-D_{2}, \quad \text { where } \quad-C_{2}=\min \left(\frac{s_{22}}{s_{23}}\right) \quad \text { and } \quad-D_{2}=\left(\lambda^{2}+1\right) \min \left(-\frac{s_{21}}{s_{23}}\right)
$$

and the second one tells us that

$$
\nu_{j}^{2} \geq C_{1} \mu_{m}^{2}+D_{1}, \quad \text { where } \quad C_{1}=\min \left(-\frac{s_{32}}{s_{33}}\right) \quad \text { and } \quad D_{1}=\left(\lambda^{2}+1\right) \min \left(\frac{s_{31}}{s_{33}}\right) .
$$

Since $\left(\nu_{j}^{2}\right)_{m \leq j \leq m+k-1}$ is the set of eigenvalues of 2.37 and 2.38 , for a fixed $\mu_{m}^{2}$ of multiplicity $k$ we obtain from these estimates that

$$
C_{1} \mu_{m}^{2}+D_{1} \leq \nu_{j}^{2} \leq C_{2} \mu_{m}^{2}+D_{2}, \quad \forall m \leq j \leq m+k-1 .
$$

In other words, thanks to our numerotation of the coupled spectrum explained in Remark 2.6 .

$$
C_{1} \mu_{m}^{2}+D_{1} \leq \nu_{m}^{2} \leq C_{2} \mu_{m}^{2}+D_{2}, \quad \forall m \geq 1 .
$$

Remark 2.10. 1. Thanks to the condition given in Remark 1.18 ,

$$
C_{1}=\min \left(-\frac{s_{32}}{s_{33}}\right)<-\min \left(\frac{s_{22}}{s_{23}}\right)=C_{2} .
$$

2. The previous Lemma says that the coupled spectrum $\left\{\left(\mu_{m}^{2}, \nu_{m}^{2}\right), m \geq 1\right\}$ lives in a cone contained in the quadrant $\left(\mathbb{R}^{+}\right)^{2}$ (up to a possible shift dues to the presence of the constants $D_{1}$ and $D_{2}$ ). Moreover, since the multiplicity of $\mu_{m}^{2}$ is finite for all $m \geq 1$, there is a finite number of points of the coupled spectrum on each vertical line. We can summarize these facts with the following generic picture: 


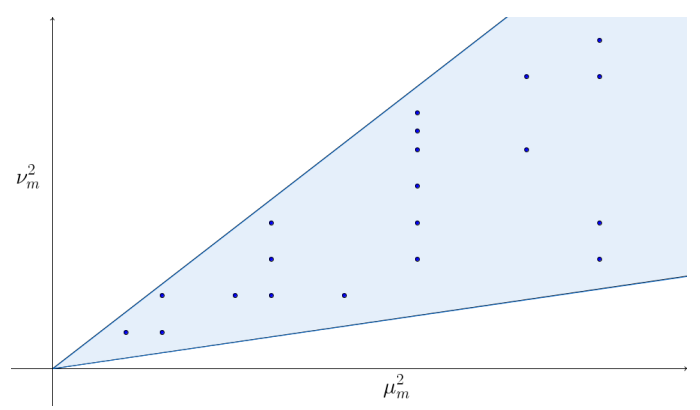

Figure 1: Coupled spectrum

3. The Weyl law (see [46] Theorem 2.21) which says (in two dimensions) that there exists a constant $C$ such that the eigenvalues are equivalent for large $m$ to $C m$, is satisfied by the eigenvalues $\left\{\mu_{m}^{2}\right\}_{m}$ and $\left\{\nu_{m}^{2}\right\}_{m}$ but we have to order these sequences in an increasing order to use it. However, we ordered the coupled spectrum in such a way that the order for the $\left(\nu_{m}^{2}\right)$ is not necessary increasing.

4. An eigenvalue of the coupled spectrum $\left(\mu_{m}^{2}, \nu_{m}^{2}\right)$ has at most multiplicity four as it was mentionned in Remark 2.7.

Example 2.1. We can illustrate the notion of coupled spectrum on the examples given in Example 1.2.

1. We define the Stäckel matrix

$$
S_{1}=\left(\begin{array}{ccc}
s_{11}\left(x^{1}\right) & s_{12}\left(x^{1}\right) & s_{13}\left(x^{1}\right) \\
0 & 1 & 0 \\
0 & 0 & 1
\end{array}\right) .
$$

In this case $H=-\partial_{3}^{2}$ and $L=-\partial_{2}^{2}$ and we note that these operators can be obtained by derivation of the Killing vector fields $\partial_{2}$ and $\partial_{3}$. The coupled spectrum of these operators is $\left\{\left(m^{2}, n^{2}\right),(m, n) \in\right.$ $\left.\mathbb{Z}^{2}\right\}$ and we can decompose the space $L^{2}\left(\mathcal{T}^{2}, s^{11} d x^{2} d x^{3}\right)$ on the basis of generalized harmonics $Y_{m n}=$ $e^{i m x^{2}+i n x^{3}}$. We note that this coupled spectrum is not included in a cone strictly contained in $\left(\mathbb{R}^{+}\right)^{2}$ but there is no contradiction with Lemma 2.9 since the Stäckel matrix $S$ does not satisfies the condition (C). However, we can use the invariances of Proposition 1.16 to come down to our framework (this transformation modifies the coupled spectrum). Indeed, we can obtain the Stäckel matrix

$$
S_{2}\left(\begin{array}{ccc}
s_{11}\left(x^{1}\right) & s_{12}\left(x^{1}\right) & s_{13}\left(x^{1}\right) \\
a & b & c \\
d & e & f
\end{array}\right),
$$

where $s_{11}, s_{12}$ and $s_{13}$ are smooth functions of $x^{1}$ and $a, b, c, d, e$ and $f$ are real constants such that $b, f<0$ and $c, e>0$. In this case we have

$$
H=-\frac{s_{32}}{s^{11}} \partial_{2}^{2}+\frac{s_{22}}{s^{11}} \partial_{3}^{2}=-\frac{e}{b f-c e} \partial_{2}^{2}+\frac{b}{b f-c e} \partial_{3}^{2}
$$

and

$$
L=\frac{s_{33}}{s^{11}} \partial_{2}^{2}-\frac{s_{23}}{s^{11}} \partial_{3}^{2}=\frac{f}{b f-c e} \partial_{2}^{2}-\frac{c}{b f-c e} \partial_{3}^{2} .
$$


Thus, the coupled spectrum of the operators $H$ and $L$ can be computed using the same procedure as the one used for $S_{1}$.

We emphasize that in the case of the Stäckel matrix $S_{1}$ the coupled spectrum is in fact uncoupled. We can thus freeze one angular momentum and let the other one move on the integers. After the use of the invariance to come down to our framework these vertical or horizontal half-lines are transformed into half-lines contained in our cone of $\left(\mathbb{R}^{+}\right)^{2}$. This allows us to use the usual Complexification of the Angular Momentum method in one dimension on a half-line contained in our cone.

2. We define the Stäckel matrix

$$
S=\left(\begin{array}{ccc}
s_{11}\left(x^{1}\right) & s_{12}\left(x^{1}\right) & a s_{12}\left(x^{1}\right) \\
0 & s_{22}\left(x^{2}\right) & s_{23}\left(x^{2}\right) \\
0 & s_{32}\left(x^{3}\right) & s_{33}\left(x^{3}\right)
\end{array}\right),
$$

where $s_{11}$ and $s_{12}$ are smooth functions of $x^{1}, s_{22}$ and $s_{23}$ are smooth functions of $x^{2}, s_{32}$ and $s_{33}$ are smooth functions of $x^{3}$ and $a$ is a real constant. In this case, the Helmholtz equation (2.26) can be rewritten as

$$
A_{1} f+s_{12}(L+a H) f=0 .
$$

Therefore, the separation of variables depends only on a single angular operator given by $L+a H$ whose properties can be easily derived from the ones for $H$ and $L$. In particular, the set of angular momenta is given by $\omega_{m}^{2}=\mu_{m}^{2}+\nu_{m}^{2}, m \geq 1$, and could be used to apply the Complexification of the Angular Momentum method. Note that, even though the spectra $\left\{\mu_{m}^{2}, \nu_{m}^{2}\right\}$ are coupled, only the spectrum $\omega_{m}^{2}$ appears in the separated radial equation.

3. In the case of the Stäckel matrix

$$
S=\left(\begin{array}{ccc}
s_{1}\left(x^{1}\right)^{2} & -s_{1}\left(x^{1}\right) & 1 \\
-s_{2}\left(x^{2}\right)^{2} & s_{2}\left(x^{2}\right) & -1 \\
s_{3}\left(x^{3}\right)^{2} & -s_{3}\left(x^{3}\right) & 1
\end{array}\right),
$$

where $s_{1}$ is a smooth function of $x^{1}, s_{2}$ is a smooth function of $x^{2}$ and $s_{3}$ is a smooth function of $x^{3}$, there is no trivial symmetry. We are thus in the general case and we have to use the general method we develop in this paper.

\subsection{A first construction of characteristic and Weyl-Titchmarsh functions}

The aim of this section is to define the characteristic and Weyl-Titchmarsh functions for the radial equation choosing $-\mu_{m}^{2}$ as the spectral parameter. We recall that the radial equation is

$$
-u^{\prime \prime}+\frac{1}{2}\left(\log \left(f_{1}\right)\right)^{\prime} u^{\prime}+\left[-\left(\lambda^{2}+1\right) s_{11}+\mu_{m}^{2} s_{12}+\nu_{m}^{2} s_{13}\right] u=0,
$$

where the functions depend only on $x^{1}$. We choose $-\mu^{2}:=-\mu_{m}^{2}$ to be the spectral parameter. As mentioned in the Introduction, to do this we make a Liouville change of variables:

$$
X^{1}=g\left(x^{1}\right)=\int_{0}^{x^{1}} \sqrt{s_{12}(t)} \mathrm{d} t
$$


and we define

$$
u\left(X^{1}, \mu^{2}, \nu^{2}\right)=u\left(h\left(X^{1}\right), \mu^{2}, \nu^{2}\right),
$$

where $h=g^{-1}$ is the inverse function of $g$ and $\nu^{2}:=\nu_{m}^{2}$. In a second time, to cancel the resulting first order term and obtain a Schrödinger equation, we introduce a weight function. Precisely, we define

$$
U\left(X^{1}, \mu^{2}, \nu^{2}\right)=\left(\frac{f_{1}}{s_{12}}\left(h\left(X^{1}\right)\right)\right)^{-\frac{1}{4}} u\left(h\left(X^{1}\right), \mu^{2}, \nu^{2}\right) .
$$

After calculation, we obtain that $U\left(X^{1}, \mu^{2}, \nu^{2}\right)$ satisfies, in the variable $X^{1}$, the Schrödinger equation

$$
-\ddot{U}\left(X^{1}, \mu^{2}, \nu^{2}\right)+q_{\nu^{2}}\left(X^{1}, \lambda\right) U\left(X^{1}, \mu^{2}, \nu^{2}\right)=-\mu^{2} U\left(X^{1}, \mu^{2}, \nu^{2}\right),
$$

where,

$$
q_{\nu^{2}}\left(X^{1}, \lambda\right)=-\left(\lambda^{2}+1\right) \frac{s_{11}\left(X^{1}\right)}{s_{12}\left(X^{1}\right)}+\nu^{2} \frac{s_{13}\left(X^{1}\right)}{s_{12}\left(X^{1}\right)}+\frac{1}{16}\left(\left(\log \left(\frac{\dot{f_{1}\left(X^{1}\right)}}{s_{12}\left(X^{1}\right)}\right)\right)\right)^{2}-\frac{1}{4}\left(\log \left(\frac{\ddot{f_{1}\left(X^{1}\right)}}{s_{12}\left(X^{1}\right)}\right)\right) .
$$

with $\dot{f}=\frac{d f}{d X^{1}}, f_{1}\left(X^{1}\right):=f_{1}\left(h_{1}\left(X^{1}\right)\right), s_{11}\left(X^{1}\right):=s_{11}\left(h_{1}\left(X^{1}\right)\right), s_{12}\left(X^{1}\right):=s_{12}\left(h_{1}\left(X^{1}\right)\right)$ and $s_{13}\left(X^{1}\right):=$ $s_{13}\left(h_{1}\left(X^{1}\right)\right)$.

Lemma 2.11. The potential $q_{\nu^{2}}$ satisfies, at the end $\left\{X^{1}=0\right\}$,

$$
q_{\nu^{2}}\left(X^{1}, \lambda\right)=-\frac{\lambda^{2}+\frac{1}{4}}{\left(X^{1}\right)^{2}}+q_{0, \nu^{2}}\left(X^{1}, \lambda\right),
$$

where $X^{1} q_{0, \nu^{2}}\left(X^{1}, \lambda\right) \in L^{1}\left(0, \frac{A^{1}}{2}\right)$ with $A^{1}=g(A)$. Similarly, at the end $\left\{X^{1}=A^{1}\right\}$,

$$
q_{\nu^{2}}\left(X^{1}, \lambda\right)=-\frac{\lambda^{2}+\frac{1}{4}}{\left(A^{1}-X^{1}\right)^{2}}+q_{A^{1}, \nu^{2}}\left(X^{1}, \lambda\right),
$$

where $\left(A^{1}-X^{1}\right) q_{A^{1}, \nu^{2}}\left(X^{1}, \lambda\right) \in L^{1}\left(\frac{A^{1}}{2}, A^{1}\right)$.

Proof. We first note that since $s_{12}\left(x^{1}\right) \sim 1$ when $x^{1} \rightarrow 0$ we obtain by definition of $X^{1}$ that $X^{1} \sim x^{1}$, as $x^{1} \rightarrow 0$. Thus we can use the hyperbolicity conditions $(1.9)$ directly in the variable $X^{1}$. The lemma is then a consequence of these conditions.

We now follow the paper 22 to define the characteristic and the Weyl-Titchmarsh functions associated with Equation 2.45 . To do that, we introduce two fundamental systems of solutions $S_{j n}, j \in\{1,2\}$ and $n \in\{0,1\}$, defined by

1. When $X^{1} \rightarrow 0$,

$$
S_{10}\left(X^{1}, \mu^{2}, \nu^{2}\right) \sim\left(X^{1}\right)^{\frac{1}{2}-i \lambda} \quad \text { and } \quad S_{20}\left(X^{1}, \mu^{2}, \nu^{2}\right) \sim \frac{1}{2 i \lambda}\left(X^{1}\right)^{\frac{1}{2}+i \lambda}
$$

and when $X^{1} \rightarrow A^{1}$,

$$
S_{11}\left(X^{1}, \mu^{2}, \nu^{2}\right) \sim\left(A^{1}-X^{1}\right)^{\frac{1}{2}-i \lambda} \quad \text { and } \quad S_{21}\left(X^{1}, \mu^{2}, \nu^{2}\right) \sim-\frac{1}{2 i \lambda}\left(A^{1}-X^{1}\right)^{\frac{1}{2}+i \lambda} .
$$


2. $W\left(S_{1 n}, S_{2 n}\right)=1$ for $n \in\{0,1\}$.

3. For all $X^{1} \in\left(0, A^{1}\right), \mu \mapsto S_{j n}\left(X^{1}, \mu^{2}, \nu^{2}\right)$ is an entire function for $j \in\{1,2\}$ and $n \in\{0,1\}$.

As in [22, 32, we add singular boundary conditions at the ends $\left\{X^{1}=0\right\}$ and $\left\{X^{1}=A^{1}\right\}$ and we consider 2.45 as an eigenvalue problem. Precisely we consider the conditions

$$
U(u):=W\left(S_{10}, u\right)_{\mid X^{1}=0}=0 \quad \text { and } \quad V(u):=W\left(S_{11}, u\right)_{\mid X^{1}=A^{1}}=0,
$$

where $W(f, g)=f g^{\prime}-f^{\prime} g$ is the Wronskian of $f$ and $g$. Finally, we can define the characteristic functions

$$
\Delta_{q_{\nu^{2}}}\left(\mu^{2}\right)=W\left(S_{11}, S_{10}\right) \quad \text { and } \quad \delta_{q_{\nu^{2}}}\left(\mu^{2}\right)=W\left(S_{11}, S_{20}\right)
$$

and the Weyl-Titchmarsh function

$$
M_{q_{\nu^{2}}}\left(\mu^{2}\right)=-\frac{W\left(S_{11}, S_{20}\right)}{W\left(S_{11}, S_{10}\right)}=-\frac{\delta_{q_{\nu^{2}}}\left(\mu^{2}\right)}{\Delta_{q_{\nu^{2}}}\left(\mu^{2}\right)} .
$$

Remark 2.12. The Weyl-Titchmarsh function is the unique function such that the solution of 2.45) given by

$$
\Phi\left(X^{1}, \mu^{2}, \nu^{2}\right)=S_{10}\left(X^{1}, \mu^{2}, \nu^{2}\right)+M_{q_{\nu^{2}}}\left(\mu^{2}\right) S_{10}\left(X^{1}, \mu^{2}, \nu^{2}\right),
$$

satisfies the boundary condition at the end $\left\{X^{1}=A^{1}\right\}$.

An immediate consequence of the third condition in the definition of the fundamental systems of solutions is the following lemma.

Lemma 2.13. For any fixed $\nu$, the maps

$$
\mu \mapsto \Delta_{q_{\nu} 2}\left(\mu^{2}\right) \quad \text { and } \quad \mu \mapsto \delta_{q_{\nu} 2}\left(\mu^{2}\right)
$$

are entire.

In the following (see Subsections 2.4 and 2.5), we will define other characteristic and Weyl-Titchmarsh functions which correspond to other choices of spectral parameters which are $-\nu_{m}^{2}$ and $-\left(\mu_{m}^{2}+\nu_{m}^{2}\right)$. We study here the influence of these other choices.

Proposition 2.14. Let $\breve{X}^{1}=\breve{g}\left(x^{1}\right)$ be a change of variables and $\breve{p}$ be a weight function, then

$$
U\left(X^{1}, \mu^{2}, \nu^{2}\right)=\frac{p\left(h\left(X^{1}\right)\right)}{\breve{p}\left(\breve{h}\left(\breve{X}^{1}\right)\right)} \breve{U}\left((\breve{g} \circ h)\left(X^{1}\right), \mu^{2}, \nu^{2}\right)
$$

and

$$
V\left(X^{1}, \mu^{2}, \nu^{2}\right)=\frac{p\left(h\left(X^{1}\right)\right)}{\breve{p}\left(\breve{h}\left(\breve{X}^{1}\right)\right)} \breve{V}\left((\breve{g} \circ h)\left(X^{1}\right), \mu^{2}, \nu^{2}\right)
$$

where

$$
\begin{gathered}
p\left(h\left(X^{1}\right)\right)=\left(\frac{f_{1}}{s_{12}}\left(h\left(X^{1}\right)\right)\right)^{-\frac{1}{4}}, \\
\breve{U}\left(\breve{X}^{1}, \mu^{2}, \nu^{2}\right)=\breve{p}\left(\breve{h}\left(\breve{X}^{1}\right)\right) u\left(\breve{h}\left(\breve{X}^{1}\right), \mu^{2}, \nu^{2}\right),
\end{gathered}
$$

and

$$
\breve{V}\left(\breve{X}^{1}, \mu^{2}, \nu^{2}\right)=\breve{p}\left(\breve{h}\left(\breve{X}^{1}\right)\right) v\left(\breve{h}\left(\breve{X}^{1}\right), \mu^{2}, \nu^{2}\right)
$$

Moreover,

$$
W_{X^{1}}(U, V)=\left(\frac{p\left(h\left(X^{1}\right)\right)}{\breve{p}\left(\breve{h}\left(\breve{X}^{1}\right)\right)}\right)^{2} \partial_{X^{1}}(\breve{g} \circ h)\left(X^{1}\right) W_{\breve{X}^{1}}(\breve{U}, \breve{V}) .
$$


Corollary 2.15. Let $\hat{X}^{1}$ and $\check{X}^{1}$ be two Liouville change of variables defined by

$$
\hat{X}^{1}=\hat{g}\left(x^{1}\right)=\int_{0}^{x^{1}} \sqrt{s_{13}(t)} \mathrm{d} t \quad \text { and } \quad \check{X}^{1}=\check{g}\left(x^{1}\right)=\int_{0}^{x^{1}} \sqrt{r_{\mu^{2}, \nu^{2}}(t)} \mathrm{d} t
$$

where

$$
r_{\mu^{2}, \nu^{2}}\left(x^{1}\right):=\frac{\mu^{2} s_{12}\left(x^{1}\right)+\nu^{2} s_{13}\left(x^{1}\right)}{\mu^{2}+\nu^{2}},
$$

and let $\hat{p}$ and $\check{p}$ be two weight functions defined by

$$
\hat{p}\left(\hat{h}\left(\hat{X}^{1}\right)\right)=\left(\frac{f_{1}}{s_{13}}\left(\hat{h}\left(\hat{X}^{1}\right)\right)\right)^{-\frac{1}{4}} \quad \text { and } \quad \check{p}\left(\check{h}\left(\check{X}^{1}\right)\right)=\left(\frac{f_{1}}{r_{\mu^{2}, \nu^{2}}}\left(\check{h}\left(\check{X}^{1}\right)\right)\right)^{-\frac{1}{4}} .
$$

Let $\hat{U}$ and $\hat{V}$ be defined as

$$
\hat{U}\left(\hat{X}^{1}, \mu^{2}, \nu^{2}\right)=\hat{p}\left(\hat{h}\left(\hat{X}^{1}\right)\right) u\left(\hat{h}\left(\hat{X}^{1}\right), \mu^{2}, \nu^{2}\right) \quad \text { and } \quad \hat{V}\left(\hat{X}^{1}, \mu^{2}, \nu^{2}\right)=\hat{p}\left(\hat{h}\left(\hat{X}^{1}\right)\right) v\left(\hat{h}\left(\hat{X}^{1}\right), \mu^{2}, \nu^{2}\right) .
$$

and $\check{U}$ and $\check{V}$ be defined as

$$
\check{U}\left(\check{X}^{1}, \mu^{2}, \nu^{2}\right)=\check{p}\left(\check{h}\left(\check{X}^{1}\right)\right) u\left(\check{h}\left(\check{X}^{1}\right), \mu^{2}, \nu^{2}\right) \quad \text { and } \quad \check{V}\left(\check{X}^{1}, \mu^{2}, \nu^{2}\right)=\check{p}\left(\check{h}\left(\check{X}^{1}\right)\right) v\left(\check{h}\left(\check{X}^{1}\right), \mu^{2}, \nu^{2}\right) .
$$

Then,

$$
W_{X^{1}}(U, V)=W_{\hat{X}^{1}}(\hat{U}, \hat{V})=W_{\check{X}^{1}}(\check{U}, \check{V}) .
$$

Remark 2.16. We will use $\hat{X}^{1}$ and $\hat{p}$ in Subsection 2.4 to obtain holomorphic properties and good estimates in the variable $\nu^{2}$. Secondly, we will use $\check{X}^{1}$ and $\check{p}$ in Subsection 2.5 to show that the characteristic functions are bounded for $(\mu, \nu) \in(i \mathbb{R})^{2}$.

\subsection{Link between the scattering coefficients and the Weyl-Titchmarsh and characteristic functions}

In this Section, we follow 22, Section 3.3, and we make the link between the transmission and the reflection coefficients, corresponding to the restriction of the transmission and the reflection operators on each generalized harmonics, and the characteristic and Weyl-Titchmarsh functions defined in Subsection 2.2. First, we observe that the scattering operator defined in Theorem 1.24 leaves invariant the span of each generalized harmonic $Y_{m}$. Hence, it suffices to calculate the scattering operator on each vector space generated by the $Y_{m}$ 's. To do this, we recall from Theorem 1.24 that, given any solution $f=$ $u_{m}\left(x^{1}\right) Y_{m}\left(x^{2}, x^{3}\right) \in \mathcal{B}^{\star}$ of 2.26 , there exists a unique $\psi_{m}^{( \pm)}=\left(\psi_{0 m}^{ \pm}, \psi_{1 m}^{( \pm)}\right) \in \mathbb{C}^{2}$ such that

$$
\begin{aligned}
u_{m}\left(x^{1}\right) \simeq & \omega_{-}(\lambda)\left(\chi_{0}\left(x^{1}\right)^{\frac{1}{2}+i \lambda} \psi_{0 m}^{(-)}+\chi_{1}\left(A-x^{1}\right)^{\frac{1}{2}+i \lambda} \psi_{1 m}^{(-)}\right) \\
& -\omega_{+}(\lambda)\left(\chi_{0}\left(x^{1}\right)^{\frac{1}{2}-i \lambda} \psi_{0 m}^{(+)}+\chi_{1}\left(A-x^{1}\right)^{\frac{1}{2}-i \lambda} \psi_{1 m}^{(+)}\right),
\end{aligned}
$$

where $\omega_{ \pm}$are given by (1.16) and the cutoff functions $\chi_{0}$ and $\chi_{1}$ are defined in (1.13). As in [22], we would like to apply this result to the FSS $\left\{S_{j n}, j=1,2, n=0,1\right\}$. However we recall that $S_{j n}$ are solutions of Equation (2.45) and this Schrödinger equation was obtained from the Helmholtz equation 2.26 by a change a variables and the introduction of a weight function (see (2.44). We thus apply the previous result to the functions

$$
u_{j n}\left(x^{1}, \mu^{2}, \nu^{2}\right)=\left(\frac{f_{1}}{s_{12}}\left(x^{1}\right)\right)^{\frac{1}{4}} S_{j n}\left(g\left(x^{1}\right), \mu^{2}, \nu^{2}\right), \quad j \in\{1,2\}, \quad n \in\{0,1\} .
$$

We first study the behaviour of the weight function at the two ends in the following Lemma. 
Lemma 2.17. When $x^{1} \rightarrow 0$,

$$
\left(\frac{f_{1}}{s_{12}}\left(x^{1}\right)\right)^{\frac{1}{4}}=\sqrt{x^{1}}[1]_{\epsilon_{0}}
$$

where

$$
[1]_{\epsilon_{0}}=1+O\left(\left(1+\left|\log \left(x^{1}\right)\right|\right)^{-1-\epsilon_{0}}\right) .
$$

The corresponding result at the end $\left\{x^{1}=A\right\}$ is also true.

Proof. We first divide the Robertson condition (1.6) by $s_{12}$ and we obtain that

$$
\frac{f_{1}}{s_{12}}=\frac{\left(\frac{\operatorname{det}(S)}{s_{12}}\right)^{2}}{\frac{H_{1}^{2}}{s_{12}} H_{2}^{2} H_{3}^{2}} .
$$

We use the hyperbolicity conditions given in 1.8 - 1.9 and Remark 1.22 to obtain that

$$
\left\{\begin{array}{l}
\frac{\operatorname{det}(S)}{s_{12}}=\frac{s^{11}}{\left(x^{1}\right)^{2}}[1]_{\epsilon_{0}} \\
\frac{H_{1}^{2}}{s_{12}}=\frac{1}{\left(x^{1}\right)^{2}}[1]_{\epsilon_{0}} \\
H_{2}^{2}=\frac{s^{11}}{\left(x^{1}\right)^{2}}[1]_{\epsilon_{0}} \\
H_{3}^{2}=\frac{s^{11}}{\left(x^{1}\right)^{2}}[1]_{\epsilon_{0}}
\end{array} .\right.
$$

The Lemma is then a direct consequence of these estimates.

Thanks to 2.47-2.48, 2.53 and Lemma 2.17, we obtain that when $x^{1} \rightarrow 0$,

$$
u_{10}\left(x^{1}, \mu^{2}, \nu^{2}\right) \sim\left(x^{1}\right)^{1-i \lambda} \quad \text { and } \quad u_{20}\left(x^{1}, \mu^{2}, \nu^{2}\right) \sim \frac{1}{2 i \lambda}\left(x^{1}\right)^{1+i \lambda},
$$

and when $x^{1} \rightarrow A$,

$$
u_{11}\left(x^{1}, \mu^{2}, \nu^{2}\right) \sim\left(A-x^{1}\right)^{1-i \lambda} \quad \text { and } \quad u_{21}\left(x^{1}, \mu^{2}, \nu^{2}\right) \sim-\frac{1}{2 i \lambda}\left(A-x^{1}\right)^{1+i \lambda} .
$$

We denote by $\psi^{(-)}=\left(\psi_{0}^{(-)}, \psi_{1}^{(-)}\right)$and $\psi^{(+)}=\left(\psi_{0}^{(+)}, \psi_{1}^{(+)}\right)$the constants appearing in Theorem 1.24 corresponding to $u_{10}$. Since $u_{10} \sim\left(x^{1}\right)^{1-i \lambda}$ when $x^{1} \rightarrow 0$, we obtain that

$$
\psi_{0}^{(-)}=0 \quad \text { and } \quad \psi_{0}^{(+)}=-\frac{1}{\omega_{+}(\lambda)} .
$$

We now write $S_{10}$ as a linear combination of $S_{11}$ and $S_{21}$, i.e.

$$
S_{10}=a_{1}\left(\mu_{m}^{2}, \nu_{m}^{2}\right) S_{11}+b_{1}\left(\mu_{m}^{2}, \nu_{m}^{2}\right) S_{21},
$$

where

$$
a_{1}\left(\mu_{m}^{2}, \nu_{m}^{2}\right)=W\left(S_{10}, S_{21}\right) \quad \text { and } \quad b_{1}\left(\mu_{m}^{2}, \nu_{m}^{2}\right)=W\left(S_{11}, S_{10}\right) .
$$

Thus, thanks to 2.53),

$$
u_{10}=a_{1}\left(\mu_{m}^{2}, \nu_{m}^{2}\right) u_{11}+b_{1}\left(\mu_{m}^{2}, \nu_{m}^{2}\right) u_{21} .
$$

We then obtain, thanks to 2.54, that

$$
\psi_{1}^{(-)}=-\frac{b_{1}\left(\mu_{m}^{2}, \nu_{m}^{2}\right)}{2 i \lambda \omega_{-}(\lambda)} \quad \text { and } \quad \psi_{1}^{(+)}=-\frac{a_{1}\left(\mu_{m}^{2}, \nu_{m}^{2}\right)}{\omega_{+}(\lambda)} .
$$


Finally, we have shown that $u_{10}$ satisfies the decomposition of Theorem 1.24 with

$$
\psi^{(-)}=\left(\begin{array}{c}
0 \\
-\frac{b_{1}\left(\mu_{m}^{2}, \nu_{m}^{2}\right)}{2 i \lambda \omega_{-}(\lambda)}
\end{array}\right) \quad \text { and } \quad \psi^{(+)}=\left(\begin{array}{c}
-\frac{1}{\omega_{+}(\lambda)} \\
-\frac{a_{1}\left(\mu_{m}^{2}, \nu_{m}^{2}\right)}{\omega_{+}(\lambda)}
\end{array}\right) .
$$

We follow the same procedure for $u_{11}$ and we obtain the corresponding vectors

$$
\phi^{(-)}=\left(\begin{array}{c}
\frac{b_{0}\left(\mu_{m}^{2}, \nu_{m}^{2}\right)}{2 i \lambda \omega-(\lambda)} \\
0
\end{array}\right) \quad \text { and } \quad \phi^{(+)}=\left(\begin{array}{c}
-\frac{a_{0}\left(\mu_{m}^{2}, \nu_{m}^{2}\right)}{\omega_{+}(\lambda)} \\
-\frac{1}{\omega_{+}(\lambda)}
\end{array}\right),
$$

where $a_{0}\left(\mu_{m}^{2}, \nu_{m}^{2}\right)=W\left(S_{11}, S_{20}\right)$ and $b_{0}\left(\mu_{m}^{2}, \nu_{m}^{2}\right)=W\left(S_{10}, S_{11}\right)$. We now recall that for all $\psi_{m}^{(-)} \in \mathbb{C}^{2}$ there exists a unique vector $\psi_{m}^{(+)} \in \mathbb{C}^{2}$ and $u_{m}(x) Y_{m} \in \mathcal{B}^{\star}$ satisfying 2.26 for which the expansion 1.15 holds. This defines the scattering matrix $S_{g}\left(\lambda, \mu_{m}^{2}, \nu_{m}^{2}\right)$ as the $2 \times 2$ matrix such that for all $\psi_{m}^{(-)} \in \mathbb{C}^{2}$

$$
\psi_{m}^{(+)}=S_{g}\left(\lambda, \mu_{m}^{2}, \nu_{m}^{2}\right) \psi_{m}^{(-)} .
$$

Using the notation

$$
S_{g}\left(\lambda, \mu_{m}^{2}, \nu_{m}^{2}\right)=\left(\begin{array}{cc}
L\left(\lambda, \mu_{m}^{2}, \nu_{m}^{2}\right) & T_{L}\left(\lambda, \mu_{m}^{2}, \nu_{m}^{2}\right) \\
T_{R}\left(\lambda, \mu_{m}^{2}, \nu_{m}^{2}\right) & R\left(\lambda, \mu_{m}^{2}, \nu_{m}^{2}\right)
\end{array}\right)
$$

and, using the definition (2.57) of the partial scattering matrix together with 2.55)-(2.56), we find

$$
S_{g}\left(\lambda, \mu_{m}^{2}, \nu_{m}^{2}\right)=\left(\begin{array}{ll}
-\frac{2 i \lambda \omega_{-}(\lambda)}{\omega_{+}(\lambda)} \frac{a_{0}\left(\mu_{m}^{2}, \nu_{m}^{2}\right)}{b_{0}\left(\mu_{m}^{2}, \nu_{m}^{2}\right)} & \frac{2 i \lambda \omega_{-}(\lambda)}{\omega_{+}(\lambda)} \frac{1}{b_{1}\left(\mu_{m}^{2}, \nu_{m_{2}^{2}}^{2}\right)} \\
-\frac{2 i \lambda \omega_{-}(\lambda)}{\omega_{+}(\lambda)} \frac{1}{b_{0}\left(\mu_{m}^{2}, \nu_{m}^{2}\right)} & \frac{2 i \lambda \omega_{-}(\lambda)}{\omega_{+}(\lambda)} \frac{a_{1}\left(\mu_{m}^{2}, \nu_{m}^{2}\right)}{b_{1}\left(\mu_{m}^{2}, \nu_{m}^{2}\right)}
\end{array}\right) .
$$

In this expression of the partial scattering matrix, we recognize the usual transmission coefficients $T_{L}\left(\lambda, \mu_{m}^{2}, \nu_{m}^{2}\right)$ and $T_{R}\left(\lambda, \mu_{m}^{2}, \nu_{m}^{2}\right)$ and the reflection coefficients $L\left(\lambda, \mu_{m}^{2}, \nu_{m}^{2}\right)$ and $R\left(\lambda, \mu_{m}^{2}, \nu_{m}^{2}\right)$ from the left and the right respectively. Since they are written in terms of Wronskians of the $S_{j n}, j=1,2, n=0,1$, we can make the link with the characteristic function (2.50) and the generalized Weyl-Titchmarsh function 2.51) as follows. Noting that,

$$
\Delta_{q_{\nu_{m}^{2}}}\left(\mu_{m}^{2}\right)=b_{1}\left(\mu_{m}^{2}, \nu_{m}^{2}\right)=-b_{0}\left(\mu_{m}^{2}, \nu_{m}^{2}\right) \quad \text { and } \quad M_{q_{\nu_{m}^{2}}}\left(\mu_{m}^{2}\right)=\frac{a_{0}\left(\mu_{m}^{2}, \nu_{m}^{2}\right)}{b_{0}\left(\mu_{m}^{2}, \nu_{m}^{2}\right)}
$$

we get,

$$
L\left(\lambda, \mu_{m}^{2}, \nu_{m}^{2}\right)=-\frac{2 i \lambda \omega_{-}(\lambda)}{\omega_{+}(\lambda)} M_{q_{\nu_{m}^{2}}}\left(\mu_{m}^{2}\right)
$$

and

$$
T\left(\lambda, \mu_{m}^{2}, \nu_{m}^{2}\right)=T_{L}\left(\lambda, \mu_{m}^{2}, \nu_{m}^{2}\right)=T_{R}\left(\lambda, \mu_{m}^{2}, \nu_{m}^{2}\right)=\frac{2 i \lambda \omega_{-}(\lambda)}{\omega_{+}(\lambda)} \frac{1}{\Delta_{q_{\nu_{m}^{2}}}\left(\mu_{m}^{2}\right)} .
$$

Finally, using the fact that the scattering operator is unitary (see Theorem 1.24 ) we obtain as in 22 the equality

$$
R\left(\lambda, \mu_{m}^{2}, \nu_{m}^{2}\right)=\frac{2 i \lambda \omega_{-}(\lambda)}{\omega_{+}(\lambda)} \frac{\overline{\Delta_{q_{\nu_{m}^{2}}}\left(\mu_{m}^{2}\right)}}{\Delta_{q_{\nu_{m}^{2}}}\left(\mu_{m}^{2}\right)} \overline{M_{\nu_{m}^{2}}\left(\mu_{m}^{2}\right)}
$$




\subsection{A second construction of characteristic and Weyl-Titchmarsh functions}

In Subsection 2.2 we defined the characteristic and the Weyl-Titchmarsh functions when $-\mu_{m}^{2}$ is the spectral parameter. We can also define these functions when we put $-\nu_{m}^{2}$ as the spectral parameter. We recall that the radial equation is given by 2.43). To choose $-\nu^{2}:=-\nu_{m}^{2}$ as the spectral parameter we make the Liouville change of variables:

$$
\hat{X}^{1}=\hat{g}\left(x^{1}\right)=\int_{0}^{x^{1}} \sqrt{s_{13}(t)} \mathrm{d} t
$$

and we define

$$
\hat{u}\left(\hat{X}^{1}, \mu^{2}, \nu^{2}\right)=u\left(\hat{h}\left(\hat{X}^{1}\right), \mu^{2}, \nu^{2}\right),
$$

where $\hat{h}=\hat{g}^{-1}$ and $\mu^{2}:=\mu_{m}^{2}$. As in Subsection 3.1 we introduce a weight function and we define

$$
\hat{U}\left(\hat{X}^{1}, \mu^{2}, \nu^{2}\right)=\left(\frac{f_{1}}{s_{13}}\left(\hat{h}\left(\hat{X}^{1}\right)\right)\right)^{-\frac{1}{4}} u\left(\hat{h}\left(\hat{X}^{1}\right), \mu^{2}, \nu^{2}\right) .
$$

After calculation, we obtain that $\hat{U}\left(\hat{X}^{1}, \mu^{2}, \nu^{2}\right)$ satisfies, in the variable $\hat{X}^{1}$, the Schrödinger equation

$$
-\ddot{\hat{U}}\left(\hat{X}^{1}, \mu^{2}, \nu^{2}\right)+\hat{q}_{\mu^{2}}\left(\hat{X}^{1}, \lambda\right) U\left(\hat{X}^{1}, \mu^{2}, \nu^{2}\right)=-\nu^{2} U\left(\hat{X}^{1}, \mu^{2}, \nu^{2}\right),
$$

where,

$$
\hat{q}_{\mu^{2}}\left(\hat{X}^{1}, \lambda\right)=-\left(\lambda^{2}+1\right) \frac{s_{11}\left(\hat{X}^{1}\right)}{s_{13}\left(\hat{X}^{1}\right)}+\mu^{2} \frac{s_{12}\left(\hat{X}^{1}\right)}{s_{13}\left(\hat{X}^{1}\right)}+\frac{1}{16}\left(\left(\log \left(\frac{f_{1}\left(\hat{X}^{1}\right)}{s_{13}\left(\hat{X}^{1}\right)}\right)\right)\right)^{2}-\frac{1}{4}\left(\log \left(\frac{f_{1}\left(\hat{X}^{1}\right)}{s_{13}\left(\hat{X}^{1}\right)}\right)\right) .
$$

As in Subsection 2.2 we can prove the following Lemma.

Lemma 2.18. The potential $\hat{q}_{\mu^{2}}$ satisfies, at the end $\left\{\hat{X}^{1}=0\right\}$,

$$
\hat{q}_{\mu^{2}}\left(\hat{X}^{1}, \lambda\right)=-\frac{\lambda^{2}+\frac{1}{4}}{\left(\hat{X}^{1}\right)^{2}}+\hat{q}_{0, \mu^{2}}\left(\hat{X}^{1}, \lambda\right),
$$

where $\hat{X}^{1} \hat{q}_{0, \mu^{2}}\left(\hat{X}^{1}, \lambda\right) \in L^{1}\left(0, \frac{\hat{A}^{1}}{2}\right)$ with $\hat{A}^{1}=\hat{g}(A)$. Similarly, at the end $\left\{\hat{X}^{1}=\hat{A}^{1}\right\}$

$$
\hat{q}_{\mu^{2}}\left(\hat{X}^{1}, \lambda\right)=-\frac{\lambda^{2}+\frac{1}{4}}{\left(\hat{A}^{1}-\hat{X}^{1}\right)^{2}}+\hat{q}_{\hat{A}^{1}, \mu^{2}}\left(\hat{X}^{1}, \lambda\right),
$$

where $\left(\hat{A}^{1}-\hat{X}^{1}\right) \hat{q}_{\hat{A}^{1}, \mu^{2}}\left(\hat{X}^{1}, \lambda\right) \in L^{1}\left(\frac{\hat{A}^{1}}{2}, \hat{A}^{1}\right)$.

We can now follow the procedure of Subsection 2.2 to define the characteristic and Weyl-Titchmarsh functions corresponding to Equation (2.62) using two fondamental systems of solutions. Thus, we can define the characteristic functions

$$
\hat{\Delta}_{\hat{q}_{\mu^{2}}}\left(\nu^{2}\right)=W\left(\hat{S}_{11}, \hat{S}_{10}\right) \quad \text { and } \quad \hat{\delta}_{\hat{q}_{\mu^{2}}}\left(\nu^{2}\right)=W\left(\hat{S}_{11}, \hat{S}_{20}\right)
$$


and the Weyl-Titchmarsh function

$$
\hat{M}_{\hat{q}_{\mu^{2}}}\left(\nu^{2}\right)=-\frac{W\left(\hat{S}_{11}, \hat{S}_{20}\right)}{W\left(\hat{S}_{11}, \hat{S}_{10}\right)}=-\frac{\hat{\delta}_{\hat{q}_{\mu^{2}}}\left(\nu^{2}\right)}{\hat{\Delta}_{\hat{q}_{\mu^{2}}}\left(\nu^{2}\right)} .
$$

Thanks to Corollary 2.15 we immediately obtain the following Lemma.

Lemma 2.19 .

$$
\Delta_{q_{\nu_{m}^{2}}}\left(\mu_{m}^{2}\right)=\hat{\Delta}_{\hat{q}_{\mu_{m}^{2}}}\left(\nu_{m}^{2}\right) \quad \text { and } \quad M_{q_{\nu_{m}^{2}}}\left(\mu_{m}^{2}\right)=\hat{M}_{\hat{q}_{\mu_{m}^{2}}}\left(\nu_{m}^{2}\right), \quad \forall m \geq 1 .
$$

As in Subsection 2.2 the characteristic functions satisfies the following lemma.

Lemma 2.20. For any fixed $\mu$ the maps

$$
\nu \mapsto \hat{\Delta}_{\hat{q}_{\mu^{2}}}\left(\nu^{2}\right)=\Delta_{q_{\nu^{2}}}\left(\mu^{2}\right) \quad \text { and } \quad \nu \mapsto \hat{\delta}_{\hat{q}_{\mu^{2}}}\left(\nu^{2}\right)=\delta_{q_{\nu^{2}}}\left(\mu^{2}\right)
$$

are entire.

\subsection{A third construction of characteristic and Weyl-Titchmarsh functions and application}

The aim of this Subsection is to show that, if we allow the angular momenta to be complex numbers, the characteristic functions $\Delta$ and $\delta$ are bounded on $(i \mathbb{R})^{2}$. Thus, in this Subsection $\mu_{m}$ and $\nu_{m}$ are assume to be in $i \mathbb{R}$. In Subsections 2.2 and 2.4 we defined the characteristic and the Weyl-Titchmarsh functions with $-\mu_{m}^{2}$ and $-\nu_{m}^{2}$ as the spectral parameter respectively. We now make a third choice of spectral parameter. We recall that the radial equation is given by 2.43) and we rewrite this equation as

$$
-u^{\prime \prime}+\frac{1}{2}\left(\log \left(f_{1}\right)\right)^{\prime} u^{\prime}-\left(\lambda^{2}+1\right) s_{11} u=-\left(\mu_{m}^{2}+\nu_{m}^{2}\right)\left(\frac{\mu_{m}^{2} s_{12}+\nu_{m}^{2} s_{13}}{\mu_{m}^{2}+\nu_{m}^{2}}\right) u .
$$

We put, for $\left(y, y^{\prime}\right) \in \mathbb{R}^{2}$,

$$
\mu:=\mu_{m}=i y, \quad \nu:=\nu_{m}=i y^{\prime}, \quad \omega^{2}:=\mu^{2}+\nu^{2} \quad \text { and } \quad r_{\mu^{2}, \nu^{2}}\left(x^{1}\right):=\frac{\mu^{2} s_{12}\left(x^{1}\right)+\nu^{2} s_{13}\left(x^{1}\right)}{\mu^{2}+\nu^{2}} .
$$

Remark 2.21. There exist some positive constants $c_{1}$ and $c_{2}$ such that for all $(\mu, \nu) \in(i \mathbb{R})^{2}$ and $x^{1} \in$ $(0, A)$,

$$
0<c_{1} \leq r_{\mu^{2}, \nu^{2}}\left(x^{1}\right) \leq c_{2}<+\infty .
$$

To choose $-\omega^{2}$ as the spectral parameter we make a Liouville change of variables (that depends on $\mu^{2}$ and $\nu^{2}$ and is a kind of average of the previous ones):

$$
\check{X}_{\mu^{2}, \nu^{2}}^{1}=\check{g}_{\mu^{2}, \nu^{2}}\left(x^{1}\right)=\int_{0}^{x^{1}} \sqrt{r_{\mu^{2}, \nu^{2}}(t)} \mathrm{d} t .
$$

For the sake of clarity, we put

$$
\check{X}^{1}:=\check{X}_{\mu^{2}, \nu^{2}}^{1} \quad \text { and } \quad \check{g}\left(x^{1}\right):=\check{g}_{\mu^{2}, \nu^{2}}\left(x^{1}\right) .
$$


We define

$$
\check{u}\left(\check{X}^{1}, \mu^{2}, \nu^{2}\right)=u\left(\check{h}\left(\check{X}^{1}\right), \mu^{2}, \nu^{2}\right),
$$

where $\check{h}=\check{g}^{-1}$. As in Subsection 3.1. we introduce a weight function and we define

$$
\check{U}\left(\check{X}^{1}, \mu^{2}, \nu^{2}\right)=\left(\frac{f_{1}}{r_{\mu^{2}, \nu^{2}}}\left(\check{h}\left(\check{X}^{1}\right)\right)\right)^{-\frac{1}{4}} u\left(\check{h}\left(\check{X}^{1}\right), \mu^{2}, \nu^{2}\right) .
$$

After calculation, we obtain that $\check{U}\left(\check{X}^{1}, \mu^{2}, \nu^{2}\right)$ satisfies, in the variable $\check{X}^{1}$, the Schrödinger equation

$$
-\ddot{\breve{U}}\left(\check{X}^{1}, \mu^{2}, \nu^{2}\right)+\check{q}_{\mu^{2}, \nu^{2}}\left(\check{X}^{1}, \lambda\right) \check{U}\left(\check{X}^{1}, \mu^{2}, \nu^{2}\right)=-\omega^{2} \check{U}\left(\check{X}^{1}, \mu^{2}, \nu^{2}\right),
$$

where,

$$
\check{q}_{\mu^{2}, \nu^{2}}\left(\check{X}^{1}, \lambda\right)=-\left(\lambda^{2}+1\right) \frac{s_{11}\left(\check{X}^{1}\right)}{r_{\mu^{2}, \nu^{2}}\left(\check{X}^{1}\right)}+\frac{1}{16}\left(\left(\log \left(\frac{\dot{f_{1}\left(\check{X}^{1}\right)}}{r_{\mu^{2}, \nu^{2}}\left(\check{X}^{1}\right)}\right)\right)\right)^{2}-\frac{1}{4}\left(\log \left(\frac{\ddot{f_{1}\left(\check{X}^{1}\right)}}{r_{\mu^{2}, \nu^{2}}\left(\check{X}^{1}\right)}\right)\right) .
$$

Lemma 2.22. The potential $\check{q}_{\mu^{2}, \nu^{2}}$ satisfies, at the end $\left\{\check{X}^{1}=0\right\}$,

$$
\check{q}_{\mu^{2}, \nu^{2}}\left(\check{X}^{1}, \lambda\right)=-\frac{\lambda^{2}+\frac{1}{4}}{\left(\check{X}^{1}\right)^{2}}+\check{q}_{0, \mu^{2}, \nu^{2}}\left(\check{X}^{1}, \lambda\right),
$$

where $\check{X}^{1} \check{q}_{0, \mu^{2}, \nu^{2}}\left(\check{X}^{1}, \lambda\right) \in L^{1}\left(0, \frac{\check{A}^{1}}{2}\right)$ with $\check{A}^{1}=\check{g}(A)$ and $\check{q}_{0, \mu^{2}, \nu^{2}}$ is uniformly bounded for $(\mu, \nu) \in(i \mathbb{R})^{2}$. Similarly, at the end $\left\{\check{X}^{1}=\check{A}^{1}\right\}$,

$$
\check{q}_{\mu^{2}, \nu^{2}}\left(\check{X}^{1}, \lambda\right)=-\frac{\lambda^{2}+\frac{1}{4}}{\left(\check{A}^{1}-\check{X}^{1}\right)^{2}}+\check{q}_{\check{A}^{1}, \mu^{2}, \nu^{2}}\left(\check{X}^{1}, \lambda\right),
$$

where $\left(\check{A}^{1}-\check{X}^{1}\right) \check{q}_{\check{A}^{1}, \mu^{2}, \nu^{2}}\left(\check{X}^{1}, \lambda\right) \in L^{1}\left(\frac{\check{A}^{1}}{2}, \check{A}^{1}\right)$ with $\check{A}^{1}=\check{g}(A)$ and $\check{q}_{\check{A}^{1}, \mu^{2}, \nu^{2}}$ is uniformly bounded for $(\mu, \nu) \in(i \mathbb{R})^{2}$.

Remark 2.23. Thanks to Remark 2.21, we immediately obtain that there exist some positive constants $A^{-}$and $A^{+}$such that for all $(\mu, \nu) \in(i \mathbb{R})^{2}$,

$$
A^{-} \leq \check{A}^{1}=: \check{A}_{\mu^{2}, \nu^{2}}^{1} \leq A^{+} .
$$

Once more, we follow the procedure of Subsection 2.2 to define the characteristic and Weyl-Titchmarsh functions corresponding to Equation 2.66 using two fondamental systems of solutions $\left\{\check{S}_{j 0}\right\}_{j=1,2}$ and $\left\{\breve{S}_{j 1}\right\}_{j=1,2}$ satisfying the asymptotics $2.47-2.48$. Thus, we define the characteristic function

$$
\check{\Delta}_{\check{q}_{\mu^{2}, \nu^{2}}}\left(\omega^{2}\right)=W\left(\check{S}_{11}, \check{S}_{10}\right),
$$

and the Weyl-Titchmarsh function

$$
\check{M}_{\check{q}_{\mu^{2}, \nu^{2}}}\left(\omega^{2}\right)=-\frac{W\left(\check{S}_{11}, \check{S}_{20}\right)}{W\left(\check{S}_{11}, \check{S}_{10}\right)}=:-\frac{\check{\delta}_{\check{q}_{\mu^{2}, \nu^{2}}}\left(\omega^{2}\right)}{\check{\Delta}_{\check{q}_{\mu^{2}, \nu^{2}}}\left(\omega^{2}\right)} .
$$

As in Subsection 2.4, Lemma 2.19, we can use the Corollary 2.15 to prove the following Lemma. 


\section{Lemma 2.24 .}

$$
\Delta_{q_{\nu^{2}}}\left(\mu^{2}\right)=\check{\Delta}_{\check{q}_{\mu^{2}, \nu^{2}}}\left(\omega^{2}\right) \quad \text { and } \quad M_{q_{\nu^{2}}}\left(\mu^{2}\right)=\check{M}_{\check{q}_{\mu^{2}, \nu^{2}}}\left(\omega^{2}\right), \quad \forall(\mu, \nu) \in(i \mathbb{R})^{2} .
$$

We finish this Subsection following the proof of Proposition 3.2 of 22 and proving the following Proposition.

Proposition 2.25. For $\omega=i y$, where $\pm y \geq 0$, when $|\omega| \rightarrow \infty$,

$$
\begin{gathered}
\check{\Delta}_{\check{q}_{\mu^{2}, \nu^{2}}}\left(\omega^{2}\right)=\frac{\Gamma(1-i \lambda)^{2}}{\pi 2^{2 i \lambda}} \omega^{2 i \lambda} e^{ \pm \lambda \pi} 2 \cosh \left(\omega \check{A}^{1} \mp \lambda \pi\right)[1]_{\epsilon}, \\
\check{\delta}_{\check{q}_{\mu^{2}, \nu^{2}}}\left(\omega^{2}\right)=\frac{\Gamma(1-i \lambda) \Gamma(1+i \lambda)}{2 i \lambda \pi} 2 \cosh \left(\omega \check{A}^{1}\right)[1]_{\epsilon} \\
\check{M}_{\check{q}_{\mu^{2}, \nu^{2}}}\left(\omega^{2}\right)=-\frac{\Gamma(1+i \lambda)^{2} e^{\mp \lambda \pi} 2^{2 i \lambda}}{2 i \lambda \Gamma(1-i \lambda)} \omega^{-2 i \lambda} \frac{\cosh \left(\omega \check{A}^{1}\right)}{\cosh \left(\omega \check{A}^{1} \mp \lambda \pi\right)}[1]_{\epsilon},
\end{gathered}
$$

where $[1]_{\epsilon}=O\left(\frac{1}{(\log |\omega|)^{\epsilon}}\right)$ when $|\omega| \rightarrow \infty$ with $\epsilon=\min \left(\epsilon_{0}, \epsilon_{1}\right)$.

Proof. The only difference with Proposition 3.2 of 22 is the fact that our potential $q_{\mu^{2}, \nu^{2}}$ depends on the angular momenta $\mu^{2}$ and $\nu^{2}$. However, since $\check{q}_{0, \mu^{2}, \nu^{2}}$ is uniformly bounded for $(\mu, \nu) \in(i \mathbb{R})^{2}$, we obtain Proposition 2.25 without additional complication.

Corollary 2.26. There exists $C>0$ such that for all $(\mu, \nu) \in(i \mathbb{R})^{2}$,

$$
\left|\Delta_{q_{\nu^{2}}}\left(\mu^{2}\right)\right|=\left|\check{\Delta}_{\check{q}_{\mu^{2}, \nu^{2}}}\left(\omega^{2}\right)\right| \leq C
$$

and

$$
\left|\delta_{q_{\nu^{2}}}\left(\mu^{2}\right)\right|=\left|\check{\delta}_{\check{q}_{\mu^{2}, \nu^{2}}}\left(\omega^{2}\right)\right| \leq C .
$$

Proof. This corollary is an immediate consequence of Proposition 2.25, Remark 2.23 and the definition of $\omega^{2}=\mu^{2}+\nu^{2} \leq 0$ when $(\mu, \nu) \in(i \mathbb{R})^{2}$.

\section{The inverse problem at fixed energy for the angular equations}

The aim of this Section is to show the uniqueness of the angular part of the Stäckel matrix i.e. of the second and the third lines. First, we prove that the block

$$
\left(\begin{array}{ll}
s_{22} & s_{23} \\
s_{32} & s_{33}
\end{array}\right)
$$

is uniquely determined by the knowledge of the scattering matrix at a fixed energy using the fact that the scattering matrices act on the same space and the first invariance described in Proposition 1.16 . Secondly, we use the decomposition on the generalized harmonics and the second invariance described in Proposition 1.16 to prove the uniqueness of the coefficients $s_{21}$ and $s_{31}$. We finally show the uniqueness of the coupled spectrum which will be useful in the study of the radial part. 


\subsection{A first reduction and a first uniqueness result}

We first recall that (see 1.10 ),

$$
g=\frac{\left(d x^{1}\right)^{2}+d \Omega_{\mathcal{T}^{2}}^{2}+P\left(x^{1}, x^{2}, x^{3}, d x^{1}, d x^{2}, d x^{3}\right)}{\left(x^{1}\right)^{2}}
$$

and

$$
\tilde{g}=\frac{\left(d x^{1}\right)^{2}+\tilde{d \Omega_{\mathcal{T}^{2}}}{ }^{2}+\tilde{P}\left(x^{1}, x^{2}, x^{3}, d x^{1}, d x^{2}, d x^{3}\right)}{\left(x^{1}\right)^{2}} .
$$

Our main assumption is

$$
S_{g}(\lambda)=S_{\tilde{g}}(\lambda),
$$

where the equality holds as operators acting on $L^{2}\left(\mathcal{T}^{2}, d V o l_{d \Omega_{\mathcal{T}^{2}}} ; \mathbb{C}^{2}\right)$ with

$$
d V o l_{d \Omega_{\mathcal{T}^{2}}}=\sqrt{\operatorname{det}\left(d \Omega_{\mathcal{T}^{2}}^{2}\right)}
$$

Thus,

$$
\sqrt{\operatorname{det}\left(d \Omega_{\mathcal{T}^{2}}^{2}\right)}=\sqrt{\operatorname{det}\left(\tilde{d} \Omega_{\mathcal{T}^{2}}^{2}\right)}
$$

since these operators have to act on the same space. Since,

$$
d \Omega_{\mathcal{T}^{2}}^{2}=s^{11}\left(\left(d x^{2}\right)^{2}+\left(d x^{3}\right)^{2}\right) \quad \text { and } \quad \tilde{\Omega}_{\mathcal{T}^{2}}^{2}=\tilde{s}^{11}\left(\left(d x^{2}\right)^{2}+\left(d x^{3}\right)^{2}\right),
$$

this equality implies that

$$
s^{11}=\tilde{s}^{11} .
$$

Using Remark 1.22, we can obtain more informations from this equality. Indeed, we first note that,

$$
\begin{aligned}
s^{11} & =s_{22} s_{33}-s_{23} s_{32} \\
& =s_{22} s_{33}-\left(1+s_{22}\right)\left(1+s_{33}\right) \\
& =-1-s_{22}-s_{33} .
\end{aligned}
$$

Thus, the equality (3.70), allows us to obtain

$$
s_{22}-\tilde{s}_{22}=\tilde{s}_{33}-s_{33} .
$$

Since the left-hand side only depends on the variable $x^{2}$ and the right-hand side only depends on the variable $x^{3}$, we can deduce that there exists a constant $c \in \mathbb{R}$ such that

$$
s_{22}-\tilde{s}_{22}=c=\tilde{s}_{33}-s_{33} .
$$

Using Remark 1.22 again, we can thus write

$$
\left(\begin{array}{ll}
s_{22} & s_{23} \\
s_{32} & s_{33}
\end{array}\right)=\left(\begin{array}{ll}
\tilde{s}_{22} & \tilde{s}_{23} \\
\tilde{s}_{32} & \tilde{s}_{33}
\end{array}\right)+c\left(\begin{array}{cc}
1 & 1 \\
-1 & -1
\end{array}\right)
$$

or equivalently,

$$
\left(\begin{array}{ll}
s_{22} & s_{23} \\
s_{32} & s_{33}
\end{array}\right)=\left(\begin{array}{cc}
\tilde{s}_{22} & \tilde{s}_{23} \\
\tilde{s}_{32} & \tilde{s}_{33}
\end{array}\right) G
$$


where

$$
G=\left(\begin{array}{cc}
1-c & -c \\
c & 1+c
\end{array}\right),
$$

is a constant matrix with determinant equals to 1 . Moreover, as it was mentioned in Proposition 1.16, if $\hat{S}$ is a second Stäckel matrix such that

$$
\left(\begin{array}{ll}
s_{i 2} & s_{i 3}
\end{array}\right)=\left(\begin{array}{ll}
\hat{s}_{i 2} & \hat{s}_{i 3}
\end{array}\right) G, \quad \forall i \in\{1,2,3\},
$$

then

$$
g=\hat{g}
$$

since

$$
s^{i 1}=\hat{s}^{i 1}, \quad \forall i \in\{1,2,3\} .
$$

The presence of the matrix $G$ is then due to an invariance of the metric $g$. We can thus assume that $G=I_{2}$. We conclude that

$$
\left(\begin{array}{ll}
s_{22} & s_{23} \\
s_{32} & s_{33}
\end{array}\right)=\left(\begin{array}{ll}
\tilde{s}_{22} & \tilde{s}_{23} \\
\tilde{s}_{32} & \tilde{s}_{33}
\end{array}\right)
$$

\subsection{End of the inverse problem for the angular part}

The aim of this Subsection is to show that the coefficients $s_{21}$ and $s_{31}$ are uniquely defined. First, since $\left\{\tilde{Y}_{m}\right\}_{m \geq 1}$ is a Hilbertian basis of $L^{2}\left(\mathcal{T}^{2}, s^{11} d x^{2} d x^{3}\right)$, we can deduce that, for all $m \in \mathbb{N} \backslash\{0\}$, there exists a subset $E_{m} \subset \mathbb{N} \backslash\{0\}$ such that

$$
Y_{m}=\sum_{p \in E_{m}} c_{p} \tilde{Y}_{p}
$$

We recall that, thanks to 2.29,

$$
\left(\begin{array}{c}
H \\
L
\end{array}\right)=\frac{1}{s^{11}}\left(\begin{array}{cc}
s_{32} & -s_{22} \\
-s_{33} & s_{23}
\end{array}\right)\left(\begin{array}{l}
A_{2} \\
A_{3}
\end{array}\right),
$$

where $A_{j}, j \in\{2,3\}$, were defined in 2.28. Clearly,

$$
\left(\begin{array}{l}
A_{2} \\
A_{3}
\end{array}\right)=T\left(\begin{array}{l}
H \\
L
\end{array}\right)
$$

where

$$
T=-\left(\begin{array}{ll}
s_{23} & s_{22} \\
s_{33} & s_{32}
\end{array}\right)
$$

We recall that

$$
\tilde{T}=-\left(\begin{array}{cc}
\tilde{s}_{23} & \tilde{s}_{22} \\
\tilde{s}_{33} & \tilde{s}_{32}
\end{array}\right)=T
$$

We finally deduce from 3.72 that

$$
-\left(\begin{array}{c}
\partial_{2}^{2} \\
\partial_{3}^{2}
\end{array}\right)=T\left(\begin{array}{c}
H \\
L
\end{array}\right)+\left(\lambda^{2}+1\right)\left(\begin{array}{c}
s_{21} \\
s_{31}
\end{array}\right)
$$

and we then obtain that

$$
T\left(\begin{array}{c}
H \\
L
\end{array}\right)+\left(\lambda^{2}+1\right)\left(\begin{array}{l}
s_{21} \\
s_{31}
\end{array}\right)=T\left(\begin{array}{c}
\tilde{H} \\
\tilde{L}
\end{array}\right)+\left(\lambda^{2}+1\right)\left(\begin{array}{l}
\tilde{s}_{21} \\
\tilde{s}_{31}
\end{array}\right) .
$$


Lemma 3.1. For all $m \geq 1$,

$$
\tilde{H}\left(\sum_{p \in E_{m}} c_{p} \tilde{Y}_{p}\right)=\sum_{p \in E_{m}} c_{p} \tilde{H}\left(\tilde{Y}_{p}\right) \quad \text { and } \quad \tilde{L}\left(\sum_{p \in E_{m}} c_{p} \tilde{Y}_{p}\right)=\sum_{p \in E_{m}} c_{p} \tilde{L}\left(\tilde{Y}_{p}\right) .
$$

Proof. We recall that $\tilde{H}$ is selfadjoint. Thus the operator $(\tilde{H}+i)^{-1}$ is bounded. Similarly the operator $(\tilde{H}+i)^{-1} \tilde{H}$ is bounded. Thus,

$$
\begin{aligned}
(\tilde{H}+i)^{-1} \tilde{H}\left(\sum_{p \in E_{m}} c_{p} \tilde{Y}_{p}\right) & =\sum_{p \in E_{m}} c_{p}(\tilde{H}+i)^{-1} \tilde{H}\left(\tilde{Y}_{p}\right) \\
& =(\tilde{H}+i)^{-1}\left(\sum_{p \in E_{m}} c_{p} \tilde{H}\left(\tilde{Y}_{p}\right)\right)
\end{aligned}
$$

Multiplying by $(\tilde{H}+i)$ from the left we obtain the result of the Lemma. Note that the sum $\sum_{p \in E_{m}} c_{p} \tilde{H}\left(\tilde{Y}_{p}\right)$ is finite because the coefficients $c_{p}$ are sufficiently decreasing. Indeed, we note that $c_{p}=\left\langle Y_{m}, \tilde{Y}_{p}\right\rangle$ and we can use integration by parts with the help of the operator $H$ and the Weyl law on the eigenvalues to obtain the decay we want.

Remark 3.2. If $E_{m}, m \in \mathbb{N} \backslash\{0\}$, are finite then Lemma 3.1 is obvious. In fact, following the idea of [22] Proposition 4.1, we could obtain that this sets are finite using asymptotics of the Weyl-Titchmarsh function.

Applying (3.73) to the vector of generalized harmonics

$$
\left(\begin{array}{c}
Y_{m} \\
Y_{m}
\end{array}\right)=\left(\begin{array}{ll}
\sum_{p \in E_{m}} & c_{p} \tilde{Y}_{p} \\
\sum_{p \in E_{m}} c_{p} \tilde{Y}_{p}
\end{array}\right)
$$

we obtain, thanks to Lemma 3.1 and 2.34, that,

$$
\begin{aligned}
\left(T\left(\begin{array}{c}
H \\
L
\end{array}\right)+\left(\lambda^{2}+1\right)\left(\begin{array}{c}
s_{21} \\
s_{31}
\end{array}\right)\right)\left(\begin{array}{c}
Y_{m} \\
Y_{m}
\end{array}\right) & =\left(T\left(\begin{array}{c}
\mu_{m}^{2} \\
\nu_{m}^{2}
\end{array}\right)+\left(\lambda^{2}+1\right)\left(\begin{array}{c}
s_{21} \\
s_{31}
\end{array}\right)\right)\left(\begin{array}{c}
Y_{m} \\
Y_{m}
\end{array}\right) \\
& =\sum_{p \in E_{m}} c_{p}\left(T\left(\begin{array}{c}
\mu_{m}^{2} \\
\nu_{m}^{2}
\end{array}\right)+\left(\lambda^{2}+1\right)\left(\begin{array}{c}
s_{21} \\
s_{31}
\end{array}\right)\right)\left(\begin{array}{c}
\tilde{Y}_{p} \\
\tilde{Y}_{p}
\end{array}\right)
\end{aligned}
$$

and

$$
\begin{aligned}
\left(T\left(\begin{array}{c}
H \\
L
\end{array}\right)+\left(\lambda^{2}+1\right)\left(\begin{array}{c}
s_{21} \\
s_{31}
\end{array}\right)\right)\left(\begin{array}{c}
Y_{m} \\
Y_{m}
\end{array}\right) & =\sum_{p \in E_{m}} c_{p}\left(T\left(\begin{array}{c}
\tilde{H} \\
\tilde{L}
\end{array}\right)+\left(\lambda^{2}+1\right)\left(\begin{array}{c}
\tilde{s}_{21} \\
\tilde{s}_{31}
\end{array}\right)\right)\left(\begin{array}{c}
\tilde{Y}_{p} \\
\tilde{Y}_{p}
\end{array}\right) \\
& =\sum_{p \in E_{m}} c_{p}\left(T\left(\begin{array}{c}
\tilde{\mu}_{p}^{2} \\
\tilde{\nu}_{p}^{2}
\end{array}\right)+\left(\lambda^{2}+1\right)\left(\begin{array}{c}
\tilde{s}_{21} \\
\tilde{s}_{31}
\end{array}\right)\right)\left(\begin{array}{c}
\tilde{Y}_{p} \\
\tilde{Y}_{p}
\end{array}\right) .
\end{aligned}
$$

Hence,

$$
\sum_{p \in E_{m}} c_{p}\left(T\left(\begin{array}{c}
\mu_{m}^{2} \\
\nu_{m}^{2}
\end{array}\right)+\left(\lambda^{2}+1\right)\left(\begin{array}{c}
s_{21} \\
s_{31}
\end{array}\right)\right)\left(\begin{array}{c}
\tilde{Y}_{p} \\
\tilde{Y}_{p}
\end{array}\right)=\sum_{p \in E_{m}} c_{p}\left(T\left(\begin{array}{c}
\tilde{\mu}_{p}^{2} \\
\tilde{\nu}_{p}^{2}
\end{array}\right)+\left(\lambda^{2}+1\right)\left(\begin{array}{c}
\tilde{s}_{21} \\
\tilde{s}_{31}
\end{array}\right)\right)\left(\begin{array}{c}
\tilde{Y}_{p} \\
\tilde{Y}_{p}
\end{array}\right) .
$$


Since $\left\{\tilde{Y}_{p}\right\}_{p \geq 1}$ is a Hilbertian basis we deduce from this equality that for all $m \geq 1$, for all $p \in E_{m}$,

$$
T\left(\begin{array}{c}
\mu_{m}^{2} \\
\nu_{m}^{2}
\end{array}\right)+\left(\lambda^{2}+1\right)\left(\begin{array}{c}
s_{21} \\
s_{31}
\end{array}\right)=T\left(\begin{array}{c}
\tilde{\mu}_{p}^{2} \\
\tilde{\nu}_{p}^{2}
\end{array}\right)+\left(\lambda^{2}+1\right)\left(\begin{array}{c}
\tilde{s}_{21} \\
\tilde{s}_{31}
\end{array}\right) .
$$

We deduce from (3.74) that,

$$
\left(\begin{array}{c}
\tilde{\mu}_{p}^{2}-\mu_{m}^{2} \\
\tilde{\nu}_{p}^{2}-\nu_{m}^{2}
\end{array}\right)=\left(\lambda^{2}+1\right) T^{-1}\left(\begin{array}{c}
s_{21}-\tilde{s}_{21} \\
s_{31}-\tilde{s}_{31}
\end{array}\right) .
$$

Since the right-hand side is independent of $m$ and $p$, we can deduce from this equality that there exists a constant vector

$$
\left(\begin{array}{l}
c_{1} \\
c_{2}
\end{array}\right)
$$

such that

$$
\left(\begin{array}{c}
\tilde{\mu}_{p}^{2} \\
\tilde{\nu}_{p}^{2}
\end{array}\right)=\left(\begin{array}{c}
\mu_{m}^{2} \\
\nu_{m}^{2}
\end{array}\right)+\left(\begin{array}{l}
c_{1} \\
c_{2}
\end{array}\right)
$$

and

$$
\left(\begin{array}{l}
s_{21} \\
s_{31}
\end{array}\right)=\left(\begin{array}{l}
\tilde{s}_{21} \\
\tilde{s}_{31}
\end{array}\right)+\frac{1}{\lambda^{2}+1} T\left(\begin{array}{l}
c_{1} \\
c_{2}
\end{array}\right) .
$$

From 3.75 , we immediately deduce that

$$
\left\{\begin{array}{l}
s_{21}\left(x^{2}\right)=\tilde{s}_{21}\left(x^{2}\right)-C_{1} s_{23}\left(x^{2}\right)-C_{2} s_{22}\left(x^{2}\right) \\
s_{31}\left(x^{3}\right)=\tilde{s}_{31}\left(x^{3}\right)-C_{1} s_{33}\left(x^{3}\right)-C_{2} s_{32}\left(x^{3}\right)
\end{array},\right.
$$

where $C_{i}=\frac{c_{i}}{\lambda^{2}+1}$ for $i \in\{1,2\}$. We recall that

$$
g=\sum_{i=1}^{3} H_{i}^{2}\left(d x^{i}\right)^{2} \quad \text { with } \quad H_{i}^{2}=\frac{\operatorname{det}(S)}{s^{i 1}} \quad \forall i \in\{1,2,3\} .
$$

Since the minors $s^{i 1}$ only depend on the second and the third column, they don't change under the transformation given in (3.76). Thus, as mentioned in the Introduction, Proposition 1.16, recalling that the determinant of a matrix doesn't change if we add to the first column a linear combination of the second and the third columns, we conclude that the equalities $(3.76)$ describe an invariance of the metric $g$ under the definition of the Stäckel matrix $S$. We can then choose $C_{i}=0, i \in\{1,2\}$, i.e. $c_{1}=c_{2}=0$. Finally, we have shown that

$$
\left(\begin{array}{l}
s_{21} \\
s_{31}
\end{array}\right)=\left(\begin{array}{c}
\tilde{s}_{21} \\
\tilde{s}_{31}
\end{array}\right) .
$$

From the definition of the operators $L$ and $H$ given by (2.29), we deduce from (3.71) and (3.77) that

$$
H=\tilde{H} \quad \text { and } \quad L=\tilde{L} .
$$

We then conclude that these operators have the same eigenfunctions, i.e. we can choose

$$
Y_{m}=\tilde{Y}_{m}, \quad \forall m \geq 1
$$

and the same coupled spectrum

$$
\left(\begin{array}{c}
\mu_{m}^{2} \\
\nu_{m}^{2}
\end{array}\right)=\left(\begin{array}{c}
\tilde{\mu}_{m}^{2} \\
\tilde{\nu}_{m}^{2}
\end{array}\right), \quad \forall m \geq 1
$$




\section{The inverse problem at fixed energy for the radial equation}

The aim of this Section is to show that the radial part of the Stäckel matrix, i.e. the first line, is uniquely determined by the knowledge of the scattering matrix. We first use a multivariable version of the Complex Angular Momentum method to extend the equality of the Weyl-Titchmarsh functions (valid on the coupled spectrum) to complex angular momenta. In a second time, we use the Borg-Marchenko Theorem (see for instance [32, 50]) to obtain the uniqueness of the quotients

$$
\frac{s_{11}}{s_{12}} \text { and } \frac{s_{11}}{s_{13}}
$$

\subsection{Complexification of the Angular Momenta}

We recall that, thanks to our main assumption in Theorem 1.25 , 3.78)-3.79 and 2.59, we know that,

$$
M\left(\mu_{m}^{2}, \nu_{m}^{2}\right)=\tilde{M}\left(\mu_{m}^{2}, \nu_{m}^{2}\right), \quad \forall m \geq 1
$$

where

$$
M\left(\mu_{m}^{2}, \nu_{m}^{2}\right)=M_{q_{\nu_{m}^{2}}}\left(\mu_{m}^{2}\right)=M_{\hat{q}_{\mu_{m}^{2}}}\left(\nu_{m}^{2}\right)
$$

and

$$
\tilde{M}\left(\mu_{m}^{2}, \nu_{m}^{2}\right)=M_{\tilde{q}_{\nu_{m}^{2}}}\left(\mu_{m}^{2}\right)=M_{\tilde{q}_{\mu_{m}^{2}}}\left(\nu_{m}^{2}\right)
$$

The aim of this Subsection is to show that

$$
M\left(\mu^{2}, \nu^{2}\right)=\tilde{M}\left(\mu^{2}, \nu^{2}\right), \quad \forall(\mu, \nu) \in \mathbb{C}^{2} \backslash P,
$$

where $P$ is the set of points $(\mu, \nu) \in \mathbb{C}^{2}$ such that $\left(\mu^{2}, \nu^{2}\right)$ is a pole of $M$ and $\tilde{M}$, or equivalently which is a zero of $\Delta$ and $\tilde{\Delta}$. Usually, in the Complexification of the Angular Momentum method there is only one angular momentum which we complexify using uniqueness results for holomorphic functions in certain classes. In [23], there are two independent angular momenta and the authors are able to use the Complexification of the Angular Momentum method for only one angular momentum. Here, we cannot fix one angular momentum and let the other one belong to $\mathbb{C}$ since the two angular momenta are not independent (see Lemma 2.9. We thus have to complexify simultaneously the two angular momenta and we then need to use uniqueness results for multivariable holomorphic functions. Therefore, to obtain 4.81) we want to use the following result given in [9, 10].

Theorem 4.1. Let $K$ be an open cone in $\mathbb{R}^{2}$ with vertex the origin and $T(K)=\left\{z \in \mathbb{C}^{2}, \operatorname{Re}(z) \in K\right\}$. Suppose that $f$ is a bounded and an analytic function on $T(K)$. Let $E$ be a discrete subset of $K$ such that for some constant $h>0,\left|e_{1}-e_{2}\right| \geq h$, for all $\left(e_{1}, e_{2}\right) \in E$. Let $n(r)=\# E \cap B(0, r)$. Assume that $f$ vanishes on $E$. Then $f$ is identically zero if

$$
\varlimsup \frac{n(r)}{r^{2}}>0, \quad r \rightarrow+\infty .
$$

We first introduce the function

$$
\begin{aligned}
\psi: \mathbb{C}^{2} & \rightarrow \mathbb{C} \\
(\mu, \nu) & \mapsto \tilde{\Delta}\left(\mu^{2}, \nu^{2}\right) \delta\left(\mu^{2}, \nu^{2}\right)-\Delta\left(\mu^{2}, \nu^{2}\right) \tilde{\delta}\left(\mu^{2}, \nu^{2}\right)
\end{aligned}
$$

with,

$$
\delta\left(\mu^{2}, \nu^{2}\right)=\delta_{q_{\nu^{2}}}\left(\mu^{2}\right)=\delta_{\hat{q}_{\mu^{2}}}\left(\nu^{2}\right)
$$


where $\delta_{q_{\nu^{2}}}\left(\mu^{2}\right)$ and $\delta_{\hat{q}_{\mu^{2}}}\left(\nu^{2}\right)$ were defined in 2.50 and

$$
\Delta\left(\mu^{2}, \nu^{2}\right)=\Delta_{q_{\nu^{2}}}\left(\mu^{2}\right)=\Delta_{\hat{q}_{\mu^{2}}}\left(\nu^{2}\right),
$$

where $\Delta_{q_{\nu^{2}}}\left(\mu^{2}\right)$ and $\Delta_{\hat{q}_{\mu^{2}}}\left(\nu^{2}\right)$ were defined in 2.64. Our aim is then to show that $\psi$ is identically zero.

Lemma 4.2. The map $\psi$ is entire as a function of two complex variables.

Proof. We use Hartogs Theorem (see for instance [39]) which states that a function of two complex variables is holomorphic if and only if this function is holomorphic with respect to each variable separately. Indeed, thanks to Lemmas 2.13 and 2.20 , we can then immediately conclude.

To use Theorem 4.1 we need the following estimate on the function $\psi$.

Lemma 4.3. There exist some positive constants $C, A$ and $B$ such that

$$
|\psi(\mu, \nu)| \leq C e^{A|\operatorname{Re}(\mu)|+B|\operatorname{Re}(\nu)|}, \quad \forall(\mu, \nu) \in \mathbb{C}^{2} .
$$

Proof. The proof of this Lemma consists in four steps.

Step 1: We claim that for all fixed $\nu \in \mathbb{C}$ there exists a constant $C_{1}(\nu)$ such that for all $\mu \in \mathbb{C}$

$$
|\psi(\mu, \nu)| \leq C_{1}(\nu) e^{A|\operatorname{Re}(\mu)|} .
$$

To obtain this estimate we study the solutions $S_{j 0}$ and $S_{j 1}$ defined in Subsection 2.2 First, we show that for $j \in\{1,2\}$,

$$
\begin{gathered}
\left|S_{j 0}\left(X^{1}, \mu^{2}, \nu^{2}\right)\right| \leq C(\nu) \frac{e^{|\operatorname{Re}(\mu)| X^{1}}}{|\mu|^{\frac{1}{2}}}, \\
\left|S_{j 0}^{\prime}\left(X^{1}, \mu^{2}, \nu^{2}\right)\right| \leq C(\nu)|\mu|^{\frac{1}{2}} e^{|\operatorname{Re}(\mu)| X^{1}}, \\
\left|S_{j 1}\left(X^{1}, \mu^{2}, \nu^{2}\right)\right| \leq C(\nu) \frac{e^{|\operatorname{Re}(\mu)|\left(A^{1}-X^{1}\right)}}{|\mu|^{\frac{1}{2}}}, \\
\left|S_{j 1}^{\prime}\left(X^{1}, \mu^{2}, \nu^{2}\right)\right| \leq C(\nu)|\mu|^{\frac{1}{2}} e^{|\operatorname{Re}(\mu)|\left(A^{1}-X^{1}\right)} .
\end{gathered}
$$

As in [22, we can show by an iterative procedure that

$$
\left|S_{j 0}\left(X^{1}, \mu^{2}, \nu^{2}\right)\right| \leq C\left(\frac{X^{1}}{1+|\mu| X^{1}}\right)^{\frac{1}{2}} e^{|\operatorname{Re}(\mu)| X^{1}} \exp \left(\int_{0}^{X^{1}} \frac{t\left|q_{0, \nu^{2}}(t)\right|}{1+|\mu| t} d t\right) .
$$

Recall now that, thanks to the asymptotically hyperbolic structure, we have for all $X^{1} \in\left(0, X_{0}^{1}\right)$, where $X_{0}^{1} \in\left(0, A^{1}\right)$ is fixed,

$$
t\left|q_{0, \nu^{2}}(t)\right| \leq \frac{C\left(1+\nu^{2}\right)}{t(1+|\log (t)|)^{1+\epsilon_{0}}}, \quad \forall t \in(0, X) .
$$

Thus, as shown in Subsection 3.1 of [22,

$$
\int_{0}^{X^{1}} \frac{t\left|q_{0, \nu^{2}}(t)\right|}{1+|\mu| t} d t \leq\left(1+\nu^{2}\right) O\left(\frac{1}{(\log (|\mu|))^{\epsilon_{0}}}\right) .
$$


We can then conclude

$$
\left|S_{j 0}\left(X^{1}, \mu^{2}, \nu^{2}\right)\right| \leq C(\nu) \frac{e^{|\operatorname{Re}(\mu)| X^{1}}}{|\mu|^{\frac{1}{2}}} .
$$

The result on $S_{j 0}^{\prime}\left(X^{1}, \mu^{2}, \nu^{2}\right)$ is obtained similarly using the estimate on the derivative of the Green kernel given in Proposition 3.1 of [22]. By symmetry, we also obtain the corresponding estimates on $S_{j 1}\left(X^{1}, \mu^{2}, \nu^{2}\right)$ and $S_{j 1}^{\prime}\left(X^{1}, \mu^{2}, \nu^{2}\right)$. We can then conclude that

$$
\Delta\left(\mu^{2}, \nu^{2}\right)=\Delta_{q_{\nu}}\left(\mu^{2}\right)=W\left(S_{11}, S_{10}\right)
$$

and

$$
\delta\left(\mu^{2}, \nu^{2}\right)=\delta_{q_{\nu}}\left(\mu^{2}\right)=W\left(S_{11}, S_{20}\right),
$$

satisfy

$$
\left|\Delta\left(\mu^{2}, \nu^{2}\right)\right| \leq C_{1}(\nu) e^{A|\operatorname{Re}(\mu)|} \quad \text { and } \quad\left|\delta\left(\mu^{2}, \nu^{2}\right)\right| \leq C_{1}(\nu) e^{A|\operatorname{Re}(\mu)|}, \quad \forall(\mu, \nu) \in \mathbb{C}^{2} .
$$

Finally, we have shown that

$$
|\psi(\mu, \nu)| \leq C_{1}(\nu) e^{A|\operatorname{Re}(\mu)|}, \quad \forall(\mu, \nu) \in \mathbb{C}^{2} .
$$

Step 2: We can also show that for all fixed $\mu \in \mathbb{C}$ there exists a constant $C_{2}(\mu)$ such that for all $\nu \in \mathbb{C}$

$$
|\psi(\mu, \nu)| \leq C_{2}(\mu) e^{\hat{A}|\operatorname{Re}(\nu)|} .
$$

To obtain this estimate we use the strategy of the first step on Equation 2.62 with potential 2.63) introduced in Subsection 2.4.

Step 3: Thanks to Corollary 2.26, there exists a constant $C$ such that for all $\left(y, y^{\prime}\right) \in \mathbb{R}^{2}$,

$$
\left|\psi\left(i y, i y^{\prime}\right)\right| \leq C .
$$

Step 4: We finish the proof of the Lemma by the use of the Phragmén-Lindelöf Theorem (see [1] Theorem

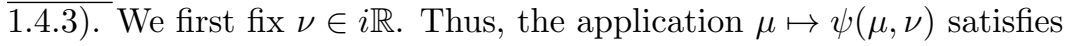

$$
\left\{\begin{array}{l}
|\psi(\mu, \nu)| \leq C_{1}(\nu) e^{A|\operatorname{Re}(\mu)|}, \quad \forall \mu \in \mathbb{C}, \quad(\text { Step 1) } \\
|\psi(\mu, \nu)| \leq C, \quad \forall \mu \in i \mathbb{R}, \quad(\text { Step 3) }
\end{array} .\right.
$$

Thanks to the Phragmén-Lindelöf Theorem, we deduce from these equalities that

$$
|\psi(\mu, \nu)| \leq C e^{A|\operatorname{Re}(\mu)|}, \quad \forall(\mu, \nu) \in \mathbb{C} \times i \mathbb{R} .
$$

We now fix $\mu \in \mathbb{C}$, then the application $\nu \mapsto \psi(\mu, \nu)$ satisfies

$$
\left\{\begin{array}{l}
|\psi(\mu, \nu)| \leq C_{2}(\mu) e^{B|\operatorname{Re}(\nu)|}, \quad \forall \nu \in \mathbb{C}, \quad(\text { Step 1) } \\
|\psi(\mu, \nu)| \leq C e^{A|\operatorname{Re}(\mu)|}, \quad \forall \nu \in i \mathbb{R}
\end{array}\right.
$$

Thus, using once more the Phragmén-Lindelöf Theorem, we obtain

$$
|\psi(\mu, \nu)| \leq C e^{A|\operatorname{Re}(\mu)|+B|\operatorname{Re}(\nu)|}, \quad \forall(\mu, \nu) \in \mathbb{C}^{2} .
$$


We apply Theorem 4.1 with

$$
K=\left(\mathbb{R}^{+}\right)^{2} \quad \text { and } \quad F(\mu, \nu)=\psi(\mu, \nu) e^{-A \mu-B \nu} .
$$

Lemma 4.4. The application $F$ is bounded and holomorphic on

$$
T\left(\left(\mathbb{R}^{+}\right)^{2}\right)=\left\{(\mu, \nu) \in \mathbb{C}^{2},(\operatorname{Re}(\mu), \operatorname{Re}(\nu)) \in \mathbb{R}^{+} \times \mathbb{R}^{+}\right\} .
$$

Proof. This lemma is an immediate consequence of Lemmas 4.2 and 4.3 .

We now recall that $\left(\mu_{m}^{2}, \nu_{m}^{2}\right), m \geq 1$, denotes the coupled spectrum of the operators $H$ and $L$. We note that $\mu_{m}^{2}$ and $\nu_{m}^{2}$ tend to $+\infty$, as $m \rightarrow+\infty$. Therefore, there exists $M \geq 1$ such that $\mu_{m}^{2} \geq 0$ and $\nu_{m}^{2} \geq 0$ for all $m \geq M$. We then set

$$
E_{M}=\left\{\left(\left|\mu_{m}\right|,\left|\nu_{m}\right|\right), m \geq M\right\} .
$$

Thanks to Equation 4.80 , we note that, the application $F$ satisfies

$$
F\left(\mu_{m}, \nu_{m}\right)=0, \quad \forall m \geq M,
$$

since

$$
\psi\left(\mu_{m}, \nu_{m}\right)=0, \quad \forall m \geq M .
$$

Moreover, since the characteristic functions are, by definition, even functions with respect to $\mu$ and $\nu$, we obtain that

$$
F\left(\left|\mu_{m}\right|,\left|\nu_{m}\right|\right)=F\left(\mu_{m}, \nu_{m}\right)=0, \quad \forall m \geq M,
$$

i.e. that $F$ vanishes on $E_{M}$.

Remark 4.5. We emphasize that $E_{M}$ denotes the set of eigenvalues counted with multiplicity (which is at most 4). Since we need a separation property given in the following Lemma to apply the Bloom's Theorem, we have to consider a new set, also denoted by $E_{M}$, which corresponds to the previous set of eigenvalues counted without multiplicity. To obtain this separation property on the coupled spectrum $E_{M}$, we also need to restrict our analysis to a suitable cone given in the following Lemma.

Lemma 4.6. We set

$$
\mathcal{C}=\left\{\left(\mu^{2}, \theta^{2} \mu^{2}\right), \quad c_{1}+\epsilon \leq \theta^{2} \leq c_{2}-\epsilon\right\}, \quad 0<\epsilon<<1,
$$

where

$$
c_{1}=\max \left(-\frac{s_{32}}{s_{33}}\right) \quad \text { and } \quad c_{2}=\min \left(-\frac{s_{22}}{s_{23}}\right) .
$$

In that case, there exists $h>0$ such that $\left|e_{1}-e_{2}\right| \geq h$ for all $\left(e_{1}, e_{2}\right) \in\left(E_{M} \cap \mathcal{C}\right)^{2}, e_{1} \neq e_{2}$.

Proof. See Appendix B.

Remark 4.7. We note that, as we have shown in Lemma 2.9, we know that there exist real constants $C_{1}, C_{2}, D_{1}$ and $D_{2}$ such that for all $m \geq 1$,

$$
C_{1} \mu_{m}^{2}+D_{1} \leq \nu_{m}^{2} \leq C_{2} \mu_{m}^{2}+D_{2},
$$

where

$$
C_{1}=\min \left(-\frac{s_{32}}{s_{33}}\right)>0 \quad \text { and } \quad C_{2}=-\min \left(\frac{s_{22}}{s_{23}}\right)>0 .
$$

We then easily obtain that

$$
0<C_{1} \leq c_{1}<c_{2} \leq C_{2}
$$


Lemma 4.8. We set

$$
n(r)=\# E_{M} \cap B(0, r) \cap \mathcal{C}
$$

where $\mathcal{C}$ was defined in 4.86 , then

$$
\varlimsup \frac{n(r)}{r^{2}}>0, \quad r \rightarrow+\infty
$$

Proof. See Appendix C

Remark 4.9. We emphasize that the number of points of the coupled spectrum $n(r)$ we use to apply the Bloom's Theorem is not exactly the one we compute in the framework of Colin de Verdière. Indeed, Colin de Verdière computes the number of points of the coupled spectrum counting multiplicity whereas $n(r)$ denotes the number of points of the coupled spectrum counting without multiplicity. However, as we have seen before (see Remark 2.7) the multiplicity of a coupled eigenvalue is at most 4 . Therefore, $n(r)$ is greater than the quarter of the number computed in the work of Colin de Verdière and is thus still of order $r^{2}$.

We can then use Theorem 4.1 on the cone $\mathcal{C}$ to conclude that

$$
F(\mu, \nu)=0, \quad \forall(\mu, \nu) \in \mathbb{C}^{2} .
$$

From this equality we immediately deduce that

$$
M\left(\mu^{2}, \nu^{2}\right)=\tilde{M}\left(\mu^{2}, \nu^{2}\right), \quad \forall(\mu, \nu) \in \mathbb{C}^{2} \backslash P,
$$

where $P$ is the set of points $(\mu, \nu) \in \mathbb{C}^{2}$ such that $\left(\mu^{2}, \nu^{2}\right)$ is a zero of the characteristic functions $\Delta$ and $\tilde{\Delta}$.

\subsection{Inverse problem for the radial part}

With the help of a multivariable version of the Complex Angular Momentum method we have shown

$$
M\left(\mu^{2}, \nu^{2}\right)=\tilde{M}\left(\mu^{2}, \nu^{2}\right), \quad \forall(\mu, \nu) \in \mathbb{C}^{2} \backslash P,
$$

where $P$ is the set of points $(\mu, \nu) \in \mathbb{C}^{2}$ such that $\left(\mu^{2}, \nu^{2}\right)$ is a zero of the characteristic function $\Delta$. By definition, it means that

$$
M_{q_{\nu^{2}}}\left(\mu^{2}\right)=M_{\tilde{q}_{\nu^{2}}}\left(\mu^{2}\right), \quad \forall(\mu, \nu) \in \mathbb{C}^{2} \backslash P,
$$

where $M_{q_{\nu 2}}\left(\mu^{2}\right)$ was defined in 2.51). We can thus use the celebrated Borg-Marchenko Theorem in the form given in [22, 32] and recalled in the Introduction (see 11.21$]-(1.22)$ ) to obtain that

$$
q_{\nu_{m}^{2}}\left(X^{1}, \lambda\right)=\tilde{q}_{\nu_{m}^{2}}\left(X^{1}, \lambda\right), \quad \forall m \geq 1, \quad \forall X^{1} \in\left(0, A^{1}\right) .
$$

Thanks to 2.46, and since the previous equality is true for all $m \geq 1$, we then have, for all $X^{1} \in\left(0, A^{1}\right)$,

$$
\frac{s_{13}\left(X^{1}\right)}{s_{12}\left(X^{1}\right)}=\frac{\tilde{s}_{13}\left(X^{1}\right)}{\tilde{s}_{12}\left(X^{1}\right)}
$$


and

$$
\begin{aligned}
& -\left(\lambda^{2}+1\right) \frac{s_{11}\left(X^{1}\right)}{s_{12}\left(X^{1}\right)}+\frac{1}{16}\left(\left(\log \left(\frac{\dot{f_{1}\left(X^{1}\right)}}{s_{12}\left(X^{1}\right)}\right)\right)\right)^{2}-\frac{1}{4}\left(\log \left(\frac{\ddot{f_{1}\left(X^{1}\right)}}{s_{12}\left(X^{1}\right)}\right)\right) \\
& =-\left(\lambda^{2}+1\right) \frac{\tilde{s}_{11}\left(X^{1}\right)}{\tilde{s}_{12}\left(X^{1}\right)}+\frac{1}{16}\left(\left(\log \left(\frac{\tilde{f}}{\tilde{s}_{12}\left(X^{1}\right)}\right)\right)\right)^{2}-\frac{1}{4}\left(\log \left(\frac{\tilde{f}_{1}\left(X^{1}\right)}{\tilde{s}_{12}\left(X^{1}\right)}\right)\right) .
\end{aligned}
$$

We want to rewrite this equation as a Cauchy problem for a second order non-linear differential equation with boundary conditions at the end $\left\{X^{1}=0\right\}$. To do that, we put

$$
f=\frac{s_{11}}{s_{12}}, \quad h=\frac{s_{12}}{f_{1}}, \quad l=\frac{s_{13}}{s_{12}}=\tilde{l} \quad \text { and } \quad u=\left(\frac{h}{\tilde{h}}\right)^{\frac{1}{4}} .
$$

We can thus rewrite 4.88 into the form

$$
u^{\prime \prime}+\frac{1}{2}(\log (\tilde{h}))^{\prime} u^{\prime}+\left(\lambda^{2}+1\right)(\tilde{f}-f) u=0 .
$$

Using the Robertson condition (1.6) we can write

$$
\frac{s_{11}}{s_{12}}=f=-\frac{s^{12}}{s^{11}}-l \frac{s^{13}}{s^{11}}+h\left(l s_{32}-s_{33}\right)\left(s_{23}-l s_{22}\right) .
$$

Remark 4.10. Thanks to 4.90, we see that we can write $\frac{s_{11}}{s_{12}}$ as a function of $\frac{s_{13}}{s_{12}}$ and $\frac{f_{1}}{s_{12}}$, i.e.

$$
\frac{s_{11}}{s_{12}}=\Phi\left(\frac{s_{13}}{s_{12}}, \frac{f_{1}}{s_{12}}\right) \quad \text { and } \quad \frac{\tilde{s}_{11}}{\tilde{s}_{12}}=\Phi\left(\frac{s_{13}}{s_{12}}, \frac{\tilde{f}_{1}}{\tilde{s}_{12}}\right),
$$

where

$$
\Phi(X, Y)=-\frac{s^{12}}{s^{11}}-X \frac{s^{13}}{s^{11}}+\frac{1}{Y}\left(X s_{32}-s_{33}\right)\left(s_{23}-X s_{22}\right) .
$$

Thus, to show that

$$
\frac{s_{11}}{s_{12}}=\frac{\tilde{s}_{11}}{\tilde{s}_{12}}
$$

it is sufficient by 4.87 to prove that

$$
\frac{f_{1}}{s_{12}}=\frac{\tilde{f}_{1}}{\tilde{s}_{12}}
$$

From 4.90, we deduce that

$$
\begin{aligned}
f-\tilde{f} & =(h-\tilde{h})\left(l s_{32}-s_{33}\right)\left(s_{23}-l s_{22}\right) \\
& =\tilde{h}\left(u^{4}-1\right)\left(l s_{32}-s_{33}\right)\left(s_{23}-l s_{22}\right) .
\end{aligned}
$$

Finally, using this last equality, we can rewrite 4.89 as

$$
u^{\prime \prime}+\frac{1}{2}(\log (\tilde{h}))^{\prime} u^{\prime}+\left(\lambda^{2}+1\right) \tilde{h}\left(l s_{32}-s_{33}\right)\left(s_{23}-l s_{22}\right)\left(u^{5}-u\right)=0 .
$$


Lemma 4.11. The function $u$ defined by

$$
u=\left(\frac{h}{\tilde{h}}\right)^{\frac{1}{4}}
$$

satisfies

$$
u(0)=1 \quad \text { and } \quad u^{\prime}(0)=0 .
$$

Proof. The proof is a consequence of the fact that the asymptotically hyperbolic structures given in the third point of Definition 1.8 are the same on the two manifolds.

We thus study the Cauchy problem

$$
\left\{\begin{array}{l}
u^{\prime \prime}+\frac{1}{2}(\log (\tilde{h}))^{\prime} u^{\prime}+\left(\lambda^{2}+1\right) \tilde{h}\left(l s_{32}-s_{33}\right)\left(s_{23}-l s_{22}\right)\left(u^{5}-u\right)=0 \\
u(0)=1 \quad \text { and } \quad u^{\prime}(0)=0
\end{array} .\right.
$$

We immediately note that $u=1$ is a solution of (4.93). By uniqueness of the Cauchy problem for the ODE 4.93 we conclude that $u=1$. We then have shown that

$$
\frac{f_{1}}{s_{12}}=\frac{\tilde{f}_{1}}{\tilde{s}_{12}}
$$

and, using 4.87) and 4.91, we can conclude that

$$
\frac{s_{11}}{s_{12}}=\frac{\tilde{s}_{11}}{\tilde{s}_{12}} \quad \text { and } \quad \frac{s_{11}}{s_{13}}=\frac{\tilde{s}_{11}}{\tilde{s}_{13}} .
$$

\section{Resolution of the inverse problem}

We can now finish the resolution of our inverse problem. We first note that

$$
g=\sum_{i=1}^{3} H_{i}^{2}\left(d x^{i}\right)^{2}=\psi^{\star} g^{\prime}
$$

where

$$
g^{\prime}=\frac{H_{1}^{2}}{s_{12}}\left(d X^{1}\right)^{2}+H_{2}^{2}\left(d x^{2}\right)^{2}+H_{3}^{2}\left(d x^{3}\right)^{2},
$$

where $\psi$ is the diffeomorphism (equal to the identity at the compactified ends $\left\{x^{1}=0\right\}$ and $\left\{x^{1}=A\right\}$ ) corresponding to the Liouville change of variables in the first variable

$$
X^{1}=\int_{0}^{x^{1}} \sqrt{s_{12}(s)} d s .
$$

Similarly,

$$
\tilde{g}=\sum_{i=1}^{3} \tilde{H}_{i}^{2}\left(d x^{i}\right)^{2}=\tilde{\psi}^{\star} \tilde{g}^{\prime}
$$

where

$$
\tilde{g}^{\prime}=\frac{\tilde{H}_{1}^{2}}{\tilde{s}_{12}}\left(d \tilde{X}^{1}\right)^{2}+\tilde{H}_{2}^{2}\left(d x^{2}\right)^{2}+\tilde{H}_{3}^{2}\left(d x^{3}\right)^{2}
$$


where $\tilde{\psi}$ is the diffeomorphism (equal to the identity at the compactified ends $\left\{x^{1}=0\right\}$ and $\left\{x^{1}=A\right\}$ ) corresponding to the same Liouville change of variables in the first variable for the second manifold. We note that, thanks to the Borg-Marchenko Theorem, we can identify

$$
A^{1}=\tilde{A}^{1} .
$$

We now note that, thanks to 4.94,

$$
\begin{aligned}
& \frac{H_{1}^{2}}{s_{12}}=\frac{\operatorname{det}(S)}{s_{12} s^{11}}=\frac{s_{11}}{s_{12}}+\frac{s^{12}}{s^{11}}+\frac{s_{13}}{s_{12}} \frac{s^{13}}{s^{11}}=\frac{\tilde{H}_{1}^{2}}{\tilde{s}_{12}}, \\
& H_{2}^{2}=\frac{\operatorname{det}(S)}{s^{21}}=\frac{\frac{s_{11}}{s_{12}} s^{11}+s^{12}+\frac{s_{13}}{s_{12}} s^{13}}{\frac{s_{13}}{s_{12}} s_{32}-s_{33}}=\tilde{H}_{2}^{2}
\end{aligned}
$$

and

$$
H_{3}^{2}=\frac{\operatorname{det}(S)}{s^{31}}=\frac{\frac{s_{11}}{s_{12}} s^{11}+s^{12}+\frac{s_{13}}{s_{12}} s^{13}}{s_{23}-\frac{s_{13}}{s_{12}} s_{22}}=\tilde{H}_{3}^{2} .
$$

We can then deduce from these equalities that

$$
g^{\prime}=\tilde{g}^{\prime}
$$

Finally, we have shown that there exists a diffeomorphism $\Psi:=\psi^{-1} \tilde{\psi}$ such that

$$
\tilde{g}=\Psi^{\star} g
$$

where $\Psi$ is the identity at the two ends. 


\section{A Proof of Proposition 1.17}

The aim of this Appendix is to prove Proposition 1.17 which we recall here.

Proposition A.1. Let $S$ be a Stäckel matrix with corresponding metric $g_{S}$. There exists a Stäckel matrix $\hat{S}$ with $g_{\hat{S}}=g_{S}$ and such that

$$
\left\{\begin{array}{lll}
\hat{s}_{12}\left(x^{1}\right)>0 \quad \text { and } \quad \hat{s}_{13}\left(x^{1}\right)>0, & \forall x^{1} \\
\hat{s}_{22}\left(x^{2}\right)<0 \quad \text { and } \quad \hat{s}_{23}\left(x^{2}\right)>0, & \forall x^{2} \\
\hat{s}_{32}\left(x^{3}\right)>0 \quad \text { and } \quad \hat{s}_{33}\left(x^{3}\right)<0, & \forall x^{3} \\
\lim _{x^{1} \rightarrow 0} s_{12}\left(x^{1}\right)=\lim _{x^{1} \rightarrow 0} s_{13}\left(x^{1}\right)=1 &
\end{array} .\right.
$$

Proof. The proof of this Proposition consists in three steps and uses the riemannian structure and the invariances of the metric described in Proposition 1.16. We first show that the coefficients of the second and the third columns are non-negative or non-positive functions. Secondly, we show that these coefficients can be assumed to be positive or negative functions. Finally, we show that we can find a Stäckel matrix with the same associated metric and satisfying the condition (C).

Step 1: We claim that for all $(i, j) \in\{1,2,3\} \times\{2,3\}, s_{i j} \geq 0$ or $s_{i j} \leq 0$. Since the proof is similar for the third column we just give the proof for the second one. First, if one of the functions $s_{12}, s_{22}$ and $s_{32}$ is identically zero, the two others cannot vanish on their intervals of definition since the minors $s^{11}$, $s^{21}$ and $s^{31}$ cannot vanish. Thus, in this case we immediately obtain that $s_{i 2} \geq 0$ or $s_{i 2} \leq 0$ for all $i \in\{1,2,3\}$. We can thus assume that there exists a triplet $\left(x_{0}^{1}, x_{0}^{2}, x_{0}^{3}\right)$ such that $s_{12}\left(x_{0}^{1}\right) \neq 0, s_{22}\left(x_{0}^{2}\right) \neq 0$ and $s_{32}\left(x_{0}^{3}\right) \neq 0$. Without loss of generality, we assume that $\operatorname{det}(S)>0$ and $s^{i 1}>0$ for all $i \in\{1,2,3\}$. From the positivity property of the minors we can deduce that, according to the sign of the quantities $s_{12}\left(x_{0}^{1}\right), s_{22}\left(x_{0}^{2}\right)$ and $s_{32}\left(x_{0}^{3}\right)$,

- $s_{12}\left(x_{0}^{1}\right)>0, s_{22}\left(x_{0}^{2}\right)>0$ and $s_{32}\left(x_{0}^{3}\right)>0$ : This case is impossible since the minors $s^{11}, s^{21}$ and $s^{31}$ cannot be all positive.

- $s_{12}\left(x_{0}^{1}\right)>0, s_{22}\left(x_{0}^{2}\right)<0$ and $s_{32}\left(x_{0}^{3}\right)>0$ :

$$
\frac{s_{33}\left(x_{0}^{3}\right)}{s_{32}\left(x_{0}^{3}\right)}<\frac{s_{23}\left(x_{0}^{2}\right)}{s_{22}\left(x_{0}^{2}\right)}<\frac{s_{13}\left(x_{0}^{1}\right)}{s_{12}\left(x_{0}^{1}\right)} .
$$

- $s_{12}\left(x_{0}^{1}\right)>0, s_{22}\left(x_{0}^{2}\right)>0$ and $s_{32}\left(x_{0}^{3}\right)<0$ :

$$
\frac{s_{13}\left(x_{0}^{1}\right)}{s_{12}\left(x_{0}^{1}\right)}<\frac{s_{33}\left(x_{0}^{3}\right)}{s_{32}\left(x_{0}^{3}\right)}<\frac{s_{23}\left(x_{0}^{2}\right)}{s_{22}\left(x_{0}^{2}\right)} .
$$

- $\underline{s_{12}\left(x_{0}^{1}\right)>0, s_{22}\left(x_{0}^{2}\right)<0 \text { and } s_{32}\left(x_{0}^{3}\right)>0:}$

$$
\frac{s_{23}\left(x_{0}^{2}\right)}{s_{22}\left(x_{0}^{2}\right)}<\frac{s_{13}\left(x_{0}^{1}\right)}{s_{12}\left(x_{0}^{1}\right)}<\frac{s_{33}\left(x_{0}^{3}\right)}{s_{32}\left(x_{0}^{3}\right)} .
$$

Since the four cases corresponding to the case $s_{12}\left(x_{0}^{1}\right)<0$ are similar, we just treat the four cases above. Assume, for instance, that there exists $\alpha_{0}^{2}$ such that $s_{22}\left(\alpha_{0}^{2}\right)=0$. We want to show that $s_{22}$ does not 
change of sign. We denote by $I$ the maximal interval (possibly reduced to $\alpha_{0}^{2}$ ) containing $\alpha_{0}^{2}$ such that $s_{22}\left(x^{2}\right)=0$ for all $x^{2} \in I$. Since the minors $s^{11}$ and $s^{31}$ are non-vanishing quantities, the functions $s_{12}$ and $s_{32}$ can then not vanish. Thus, there exists two real constants $c_{1}$ and $c_{2}$ such that

$$
c_{1} \leq \frac{s_{13}}{s_{12}} \leq c_{2} \quad \text { and } \quad c_{1} \leq \frac{s_{33}}{s_{32}} \leq c_{2}
$$

i.e. these quotients are bounded. Moreover, $s_{23}\left(x^{2}\right) \neq 0$ for all $x^{2} \in I$ and by continuity there exists an interval $J$ such that $I \subsetneq J$ and $s_{23}\left(x^{2}\right) \neq 0$ for all $x^{2} \in J$. If we assume that $s_{22}$ changes sign in a neighbourhood of $I$ we obtain that for all $\epsilon>0$ there exist $y_{0}^{2} \in J$ and $y_{1}^{2} \in J$ such that

$$
0<s_{22}\left(y_{0}^{2}\right)<\epsilon \text { and }-\epsilon<s_{22}\left(y_{1}^{2}\right)<0 .
$$

Thus, for all $M>0$ there exist, $y_{0}^{2} \in J$ and $y_{1}^{2} \in J$ such that

$$
\frac{s_{23}\left(y_{0}^{2}\right)}{s_{22}\left(y_{0}^{2}\right)}>M \quad \text { and } \quad \frac{s_{23}\left(y_{1}^{2}\right)}{s_{22}\left(y_{1}^{2}\right)}<-M .
$$

We thus obtain a contradiction between (A.98) and each of the equalities A.95, A.96) and A.97). We can then conclude that $s_{22}\left(x^{2}\right) \geq 0$ or $s_{22}\left(x^{2}\right) \leq 0$. The proof is similar for $s_{12}$ and $s_{32}$.

Step 2: We show, thanks to the first invariance given in Proposition 1.16 that there exists a Stäckel $\overline{\text { matrix }}$ having the same associated metric as $S$ and such that for all $(i, j) \in\{1,2,3\} \times\{2,3\}, s_{i j}>0$ or $s_{i j}<0$. We recall that there is at most one vanishing function $s_{i j},(i, j) \in\{1,2,3\} \times\{2,3\}$, per column since the minors $s^{11}, s^{21}$ and $s^{31}$ are non-zero quantities. We assume that one coefficient of the second column vanishes. By symmetry, we can assume that this is $s_{12}$, i.e. that $s_{12}\left(x_{0}^{1}\right)=0$ at one point $x_{0}^{1}$. We first assume that $s_{23}$ and $s_{33}$ do not vanish. In this case, there exists a real $a \geq 1$ such that

$$
\left|s_{23}\right|<a\left|s_{22}\right| \text { and }\left|s_{33}\right|<a\left|s_{32}\right|
$$

and a real constant $b \geq 1$ such that

$$
\left|s_{22}\right|<b\left|s_{23}\right| \quad \text { and } \quad\left|s_{32}\right|<b\left|s_{33}\right| \text {. }
$$

We now search a $2 \times 2$ constant invertible matrix $G$ such that the coefficients of the new Stäckel matrix, obtained by the transformation given in the first point of Proposition 1.16, are positive or negative. For instance, if $s_{12}$ and $s_{13}$ have the same sign, we put

$$
G=\left(\begin{array}{ll}
a & 1 \\
1 & b
\end{array}\right)
$$

and we thus obtain a new Stäckel matrix whose second and third columns are

$$
\left(\begin{array}{ll}
a s_{12}+s_{13} & s_{12}+b s_{13} \\
a s_{22}+s_{23} & s_{22}+b s_{23} \\
a s_{32}+s_{33} & s_{32}+b s_{33}
\end{array}\right) .
$$

We can easily show that these six components are positive or negative (we recall that $s_{12}$ and $s_{13}$ cannot vanish simultaneously). However, if $s_{12}$ and $s_{13}$ have different signs, we put

$$
G=\left(\begin{array}{cc}
a & -1 \\
-1 & b
\end{array}\right)
$$


and we also obtain positive or negative components. If $s_{23}$ or $s_{33}$ vanish we just have to choose the suitable constants $a$ and $b$ using the fact there is at most one vanishing function in the third column.

Step 3: Finally, we show, thanks to the first invariance given in Proposition 1.16 and the riemannian structure, that there exists a Stäckel matrix having the same associated metric as $S$ and satisfying the condition (C) of Definition 1.8. We recall that thanks, to the second step, we can assume that the Stäckel matrix $S$ satisfies $s_{i j}>0$ or $s_{i j}<0$ for all $(i, j) \in\{1,2,3\} \times\{2,3\}$. We recall that the metric $g$ is riemannian if and only if $\operatorname{det}(S), s^{11}, s^{21}$ and $s^{31}$ have the same sign. Without loss of generality, we assume that these quantities are all positive. We recall that according to the sign of the functions $s_{12}$, $s_{22}$ and $s_{32}$ the inequalities A.95-A.97) are satisfied. We thus have to treat different cases according to the sign of the components of the Stäckel matrix. We first want to obtain the sign conditions in (C). Since the proof are similar in the other cases, we just give the proof in the case

$$
s_{12}>0, \quad s_{22}<0 \quad \text { and } \quad s_{32}>0 .
$$

We then give, in each case, the matrix $G \in G L_{2}(\mathbb{R})$ such that the transformation given in the first invariance of Proposition 1.16 provides us the signs we want.

- If $s_{13}>0, s_{23}<0$ and $s_{33}>0$ : We put

$$
G=\left(\begin{array}{cc}
1 & -1 \\
0 & b
\end{array}\right)
$$

where

$$
\frac{s_{13}}{s_{12}}<b<\frac{s_{23}}{s_{22}}<\frac{s_{33}}{s_{32}},
$$

and we obtain the required signs. Indeed, we obtain that the second and the third column of the new Stäckel matrix are given by

$$
\left(\begin{array}{ll}
s_{12} & -s_{12}+b s_{13} \\
s_{22} & -s_{22}+b s_{23} \\
s_{32} & -s_{32}+b s_{33}
\end{array}\right)
$$

which has the desired signs thanks to our choice of constant $b$.

- If $s_{13}>0, s_{23}>0$ and $s_{33}<0:$ We put $G=I_{2}$.

- If $s_{13}>0, s_{23}<0$ and $s_{33}<0$ : We put

$$
G=\left(\begin{array}{cc}
1 & -1 \\
0 & b
\end{array}\right)
$$

where

$$
\frac{s_{33}}{s_{32}}<\frac{s_{13}}{s_{12}}<b<\frac{s_{23}}{s_{22}} .
$$

As previously, the case $s_{13}<0$ is similar and we thus omit its proof. Up to this point, we proved that we can assume that

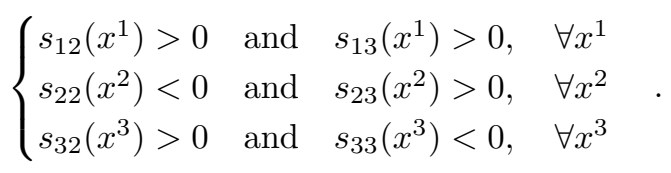


Finally, we just have to use once more the invariance with respect to the multiplication of the second and the third column by an invertible constant $2 \times 2$ matrix $G$ to obtain that we can assume that

$$
\lim _{x^{1} \rightarrow 0} s_{12}\left(x^{1}\right)=\lim _{x^{1} \rightarrow 0} s_{13}\left(x^{1}\right)=1 .
$$

Indeed, we just have to set

$$
G=\left(\begin{array}{cc}
\frac{1}{\alpha} & 0 \\
0 & \frac{1}{\beta}
\end{array}\right)
$$

where,

$$
\alpha=\lim _{x^{1} \rightarrow 0} s_{12}\left(x^{1}\right)>0 \quad \text { and } \quad \beta=\lim _{x^{1} \rightarrow 0} s_{13}\left(x^{1}\right)>0 .
$$

The result then follows. 


\section{B Proof of Lemma 4.6}

The aim of this Appendix is to prove Lemma 4.6 which we recall here.

Lemma B.1. We set

$$
E_{M}=\left\{\left(\left|\mu_{m}\right|,\left|\nu_{m}\right|\right), m \geq M\right\}
$$

and

$$
\mathcal{C}=\left\{\left(\mu^{2}, \theta^{2} \mu^{2}\right), \quad c_{1}+\epsilon \leq \theta^{2} \leq c_{2}+\epsilon\right\}, \quad 0<\epsilon<<1,
$$

where

$$
c_{1}=\max \left(-\frac{s_{32}}{s_{33}}\right) \quad \text { and } \quad c_{2}=\min \left(-\frac{s_{22}}{s_{23}}\right) .
$$

In that case, there exists $h>0$ such that $\left|e_{1}-e_{2}\right| \geq h$ for all $\left(e_{1}, e_{2}\right) \in\left(E_{M} \cap \mathcal{C}\right)^{2}, e_{1} \neq e_{2}$.

Proof. We recall that the coupled spectrum was defined in Remark 2.6 by

$$
H Y_{m}=\mu_{m}^{2} Y_{m} \quad \text { and } \quad L Y_{m}=\nu_{m}^{2} Y_{m}, \quad \forall m \geq 1,
$$

where $H$ and $L$ are commuting, elliptic and selfadjoint operators of order two. Writing $Y_{m}\left(x^{2}, x^{3}\right)=$ $v_{m}\left(x^{2}\right) w_{m}\left(x^{3}\right)$, we obtain that B.100) is equivalent to

$$
-v_{m}^{\prime \prime}\left(x^{2}\right)+\left[-\left(\lambda^{2}+1\right) s_{21}\left(x^{2}\right)+\mu_{m}^{2} s_{22}\left(x^{2}\right)+\nu_{m}^{2} s_{23}\left(x^{2}\right)\right] v_{m}\left(x^{2}\right)=0,
$$

and

$$
-w_{m}^{\prime \prime}\left(x^{3}\right)+\left[-\left(\lambda^{2}+1\right) s_{31}\left(x^{3}\right)+\mu_{m}^{2} s_{32}\left(x^{3}\right)+\nu_{m}^{2} s_{33}\left(x^{3}\right)\right] w_{m}\left(x^{3}\right)=0,
$$

where $v_{m}$ and $w_{m}$ are periodic functions, i.e.

$$
\left\{\begin{array}{l}
v_{m}(0)=v_{m}(B) \quad \text { and } \quad v_{m}^{\prime}(0)=v_{m}^{\prime}(B) \\
w_{m}(0)=w_{m}(C) \quad \text { and } \quad w_{m}^{\prime}(0)=w_{m}^{\prime}(C)
\end{array} .\right.
$$

We first consider Equation B.101 which we rewrite as

$$
-v^{\prime \prime}-\left(\lambda^{2}+1\right) s_{21} v=\mu^{2}\left[-s_{22}-\theta^{2} s_{23}\right] v,
$$

where $v:=v_{m}, \mu^{2}:=\mu_{m}^{2}, \nu^{2}:=\nu_{m}^{2}$ and

$$
\theta^{2}:=\frac{\nu^{2}}{\mu^{2}} .
$$

In the following we will consider Schrödinger equations associated with (B.101)-(B.102) whose spectral parameter is $\mu^{2}$ which tends to $+\infty$. Moreover, these equations depend on the parameter $\theta^{2}$ which is always bounded in a suitable cone we introduce now. We recall that, as we have shown in Lemma 2.9 . there exist real constants $C_{1}, C_{2}, D_{1}$ and $D_{2}$ such that for all $m \geq 1$,

$$
C_{1} \mu_{m}^{2}+D_{1} \leq \nu_{m}^{2} \leq C_{2} \mu_{m}^{2}+D_{2},
$$

where

$$
C_{1}=\min \left(-\frac{s_{32}}{s_{33}}\right)>0 \quad \text { and } \quad C_{2}=-\min \left(\frac{s_{22}}{s_{23}}\right)>0
$$


Let $\epsilon>0$ be fixed, we then consider $\theta^{2}$ such that

$$
c_{1}+\frac{D_{1}}{\mu^{2}}+\epsilon \leq \theta^{2} \leq c_{2}+\frac{D_{2}}{\mu^{2}}-\epsilon,
$$

where

$$
c_{1}=\max \left(-\frac{s_{32}}{s_{33}}\right) \quad \text { and } \quad c_{2}=\min \left(-\frac{s_{22}}{s_{23}}\right) .
$$

We note that

$$
0<C_{1} \leq c_{1}<c_{2} \leq C_{2} .
$$

This implies that, for sufficiently large $\mu^{2}$, there exists $\delta>0$ such that

$$
-s_{22}-\theta^{2} s_{23} \geq\left(\epsilon-\frac{D_{2}}{\mu^{2}}\right) s_{23} \geq \delta>0
$$

and

$$
-s_{32}-\theta^{2} s_{33} \geq\left(\epsilon+\frac{D_{1}}{\mu^{2}}\right)\left(-s_{33}\right) \geq \delta>0 .
$$

For such a $\theta^{2}$, we can thus proceed to the Liouville change of variables

$$
X^{2}=\int_{0}^{x^{2}} \sqrt{-s_{22}(t)-\theta^{2} s_{23}(t)} d t
$$

in Equation B.101). This new variable thus satisfies $X^{2} \in\left[0, \tilde{B}\left(\theta^{2}\right)\right]$, where

$$
\tilde{B}\left(\theta^{2}\right)=\int_{0}^{B} \sqrt{-s_{22}(t)-\theta^{2} s_{23}(t)} d t .
$$

Finally, we set

$$
V\left(X^{2}\right)=\left[-s_{22}\left(x^{2}\left(X^{2}\right)\right)-\theta^{2} s_{23}\left(x^{2}\left(X^{2}\right)\right)\right]^{\frac{1}{4}} v\left(x^{2}\left(X^{2}\right)\right) .
$$

This new function then satisfies in the variable $X^{2}$ the Schrödinger equation

$$
-\ddot{V}^{2}\left(X^{2}\right)+Q_{\theta^{2}}\left(X^{2}\right) V\left(X^{2}\right)=\mu^{2} V\left(X^{2}\right),
$$

where $\mu^{2}$ is the spectral parameter, $Q_{\theta^{2}}\left(X^{2}\right)$ is uniformly bounded with respect to $\theta^{2}$ satisfying B.104 and for such a $\theta^{2}$,

$$
Q_{\theta^{2}}\left(X^{2}\right)=O(1) .
$$

We now search the couples $\left(\mu^{2}, \theta^{2}\right)$ such that B.108) admits periodic solutions. We define $\left\{C_{0}, S_{0}\right\}$ and $\left\{C_{1}, S_{1}\right\}$ the usual Fondamental Systems of Solutions of (B.108), i.e.

$$
C_{0}(0)=1, \quad \dot{C}_{0}(0)=0, \quad S_{0}(0)=0 \quad \text { and } \quad \dot{S}_{0}(0)=1,
$$

and

$$
C_{1}(\tilde{B})=1, \quad \dot{C}_{1}(\tilde{B})=0, \quad S_{1}(\tilde{B})=0 \quad \text { and } \quad \dot{S}_{1}(\tilde{B})=1 .
$$

We recall that these functions are analytic and even with respect to $\mu$. We write the solutions $V$ of B.108 as

$$
V=\alpha C_{0}+\beta S_{0}=\gamma C_{1}+\delta S_{1},
$$


where $\alpha, \beta, \gamma$ and $\delta$ are real constants. Thus,

$$
V(0)=\alpha, \quad \dot{V}(0)=0, \quad V(\tilde{B})=\gamma \quad \text { and } \quad \dot{V}(\tilde{B})=\delta .
$$

$V$ is then a periodic function if and only if

$$
V(0)=V(\tilde{B}) \quad \Leftrightarrow \quad \alpha=\gamma \quad \Leftrightarrow \quad W\left(V, S_{0}\right)=W\left(V, S_{1}\right)
$$

and

$$
\dot{V}(0)=\dot{V}(\tilde{B}) \quad \Leftrightarrow \quad \beta=\delta \quad \Leftrightarrow \quad W\left(C_{0}, V\right)=W\left(C_{1}, V\right),
$$

where $W(f, g)=f g^{\prime}-f^{\prime} g$ denotes the Wronskian of two functions $f$ and $g$. In other words, $V$ is a periodic solution of $(B .108)$ if and only if

$$
W\left(V, S_{0}-S_{1}\right)=W\left(C_{0}-C_{1}, V\right)=0 .
$$

We thus add to the Equation (B.108) the boundary conditions $(\mathrm{B} .109)$ and we define the corresponding characteristic functions. In other words, we defined

$$
\Delta_{1}\left(\mu^{2}, \theta^{2}\right)=W\left(C_{0}-C_{1}, S_{0}-S_{1}\right)=2-W\left(C_{0}, S_{1}\right)-W\left(C_{1}, S_{0}\right) .
$$

We emphasize that $\Delta_{1}\left(\mu^{2}, \theta^{2}\right)$ vanishes if and only if there exists a periodic solution of $(\mathrm{B} .108)$ for $\left(\mu^{2}, \theta^{2}\right)$. The asymptotics of $W\left(C_{0}, S_{1}\right)$ and $W\left(C_{1}, S_{0}\right)$ are well known (see for instance [23, 31]). Indeed, we know that

$$
W\left(C_{0}, S_{1}\right)=\cos \left(\mu \tilde{B}\left(\theta^{2}\right)\right) \times\left(1+O\left(\frac{1}{\mu}\right)\right)
$$

and

$$
W\left(C_{1}, S_{0}\right)=\cos \left(\mu \tilde{B}\left(\theta^{2}\right)\right) \times\left(1+O\left(\frac{1}{\mu}\right)\right),
$$

where $\mu=\sqrt{\mu^{2}}$ (we do not have to precise the sign of $\mu$ since the characteristic functions are even functions). We then obtain that

$$
\Delta_{1}\left(\mu^{2}, \theta^{2}\right)=0 \quad \Leftrightarrow \quad 2-2 \cos \left(\mu \tilde{B}\left(\theta^{2}\right)\right)+O\left(\frac{1}{\mu}\right)=0 .
$$

Using the Rouché's Theorem (see for instance [31]) we can then deduce that the couples $\left(\mu^{2}, \theta^{2}\right)$ satisfying B.112 are close for large $\mu$ to the couples $\left(\mu^{2}, \theta^{2}\right)$ satisfying

$$
2-2 \cos \left(\mu \tilde{B}\left(\theta^{2}\right)\right)=0 \quad \Leftrightarrow \quad \cos \left(\mu \tilde{B}\left(\theta^{2}\right)\right)=1 .
$$

The solutions of this last equation are

$$
\mu=\frac{2 m \pi}{\tilde{B}\left(\theta^{2}\right)}, \quad m \in \mathbb{Z},
$$

for $\theta^{2}$ satisfying (B.104) and $m$ sufficiently large. Finally, we recall that $\tilde{B}\left(\theta^{2}\right)$ is given by (B.107). Thus, since $s_{23}$ is a positive function, the map $\tilde{B}$ is strictly decreasing with respect to $\theta^{2} \in\left[c_{1}+\epsilon, c_{2}-\epsilon\right]$. The map $\frac{1}{\tilde{B}\left(\theta^{2}\right)}$ is then strictly increasing. We can summarize these facts on the following picture: 


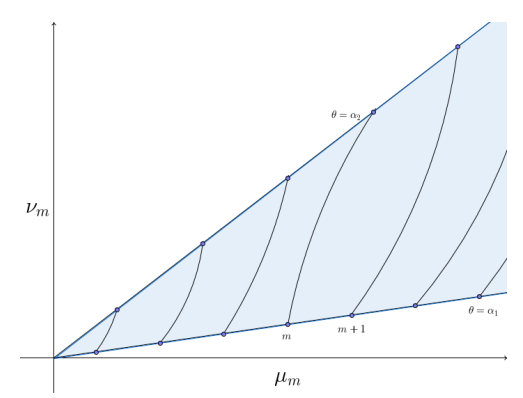

Figure 2: First approximation of the coupled spectrum

We do the same analysis on the Equation (B.102). We recall that if $\theta^{2}$ satisfies (B.104) then the inequality B.106 is satisfied for $\mu^{2}$ sufficiently large. We can thus set

$$
X^{3}=\int_{0}^{x^{3}} \sqrt{-s_{32}(t)-\theta^{2} s_{33}(t)} d t .
$$

This new variable satisfies $X^{3} \in\left[0, \tilde{C}\left(\theta^{2}\right)\right]$, where

$$
\tilde{C}\left(\theta^{2}\right)=\int_{0}^{C} \sqrt{-s_{32}(t)-\theta^{2} s_{33}(t)} d t .
$$

We then set

$$
W\left(X^{3}\right)=\left[-s_{32}\left(x^{3}\left(X^{3}\right)\right)-\theta^{2} s_{33}\left(x^{3}\left(X^{3}\right)\right)\right]^{\frac{1}{4}} w\left(x^{3}\left(X^{3}\right)\right) .
$$

This function then satisfies, in the variable $X^{3}$, the Schrödinger equation

$$
-\ddot{W}^{2}\left(X^{3}\right)+\tilde{Q}_{\theta^{2}}\left(X^{3}\right) W\left(X^{3}\right)=\mu^{2} W\left(X^{3}\right), \quad \text { where } \quad \tilde{Q}_{\theta^{2}}\left(X^{3}\right)=O(1),
$$

for $\theta^{2}$ satisfying (B.104) and $\mu^{2}$ sufficiently large. As previously, we obtain that B.114 has a periodic solution if and only if

$$
\Delta_{2}\left(\mu^{2}, \theta^{2}\right):=2-W\left(C_{0}, S_{1}\right)-W\left(C_{1}, S_{0}\right)=0 .
$$

Thanks to the asymptotics B.110 - B.111 we obtain that

$$
\Delta_{2}\left(\mu^{2}, \theta^{2}\right)=0 \quad \Leftrightarrow \quad 2-2 \cos \left(\mu \tilde{C}\left(\theta^{2}\right)\right)+O\left(\frac{1}{\mu}\right)=0 .
$$

Using once more the Rouché's Theorem, we obtain that the couples $\left(\mu^{2}, \theta^{2}\right)$ satisfying the previous equality are close for large $\mu$ to the couples satisfying

$$
\cos \left(\mu \tilde{C}\left(\theta^{2}\right)\right)=1, \quad \text { i.e. } \quad \mu^{2}=\frac{2 \pi k}{\tilde{C}\left(\theta^{2}\right)}, \quad k \in \mathbb{Z},
$$

where $k$ is sufficiently large and $\theta^{2}$ satisfies Equation B.104). We recall that $\tilde{C}\left(\theta^{2}\right)$ is given by B.113. Since $s_{33}$ is a negative function, the map $\tilde{C}$ is strictly increasing for $\theta^{2} \in\left[c_{1}+\epsilon, c_{2}-\epsilon\right]$. The map $\frac{1}{\tilde{C}\left(\theta^{2}\right)}$ is then strictly decreasing. We can summarize these facts on the following picture: 


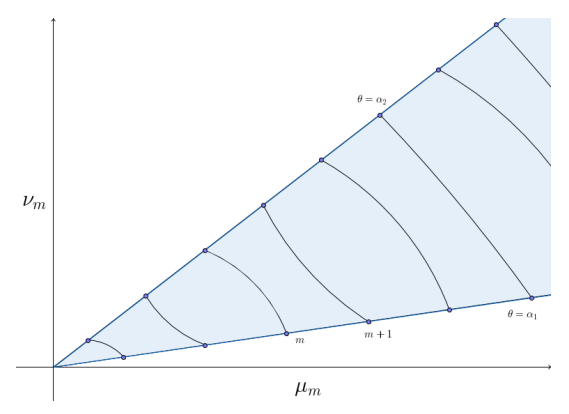

Figure 3: Second approximation of the coupled spectrum

The coupled spectrum $\Lambda=\left\{\left(\mu_{m}^{2}, \nu_{m}^{2}\right), m \geq 1\right\}$, or equivalently the coupled spectrum $\left(\mu_{m}^{2}, \theta_{m}^{2}\right)$, is then given by

$$
\Lambda=\left\{\Delta_{1}\left(\mu^{2}, \theta^{2}\right)=0\right\} \cap\left\{\Delta_{2}\left(\mu^{2}, \theta^{2}\right)=0\right\},
$$

since for all $\left(\mu_{m}^{2}, \nu_{m}^{2}\right) \in \Lambda$, there exists simultaneously a periodic solution of B.108 and a periodic solution of (B.114). Using the previous two figures we obtain the following one on which the coupled spectrum corresponds to the intersection between the previous curves:

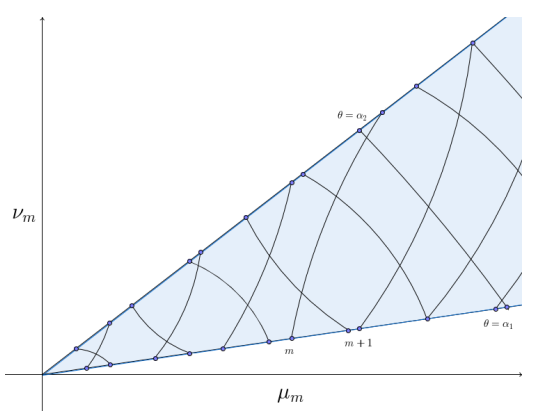

Figure 4: The coupled spectrum

We now want to use this particular structure of the coupled spectrum to prove Lemma 4.6. We work on the plane $(\mu, \theta)$ and we set $\nu=\theta \mu$, with $0<\alpha_{1} \leq \theta \leq \alpha_{2}$, where

$$
\alpha_{1}=\sqrt{c_{1}^{2}+\epsilon} \text { and } \alpha_{2}=\sqrt{c_{2}^{2}-\epsilon}
$$

with $\epsilon>0$. We recall that for large $m$ we can approximate $\mu_{m}$ by

$$
\mu_{m}=\frac{2 m \pi}{\tilde{B}\left(\theta^{2}\right)}
$$

where

$$
\tilde{B}\left(\theta^{2}\right)=\int_{0}^{B} \sqrt{-s_{22}(t)-\theta^{2} s_{23}(t)} d t .
$$

We first want to show that the curves drawn in the first Figure are uniformly separated. In other words, we show that there exists $\delta>0$ such that the distance between two successive curves is greater than $\delta$. 
Precisely, we want to show that there exists $\delta>0$ such that for large $m$ and for all $\left(\theta_{1}, \theta_{2}\right) \in\left[\alpha_{1}, \alpha_{2}\right]^{2}$,

$$
\left|\mu_{m+1}\left(\theta_{2}\right)-\mu_{m}\left(\theta_{1}\right)\right|+\left|\theta_{2} \mu_{m+1}\left(\theta_{2}\right)-\theta_{1} \mu_{m}\left(\theta_{1}\right)\right| \geq \delta .
$$

If we note,

$$
d=\left|\mu_{m+1}\left(\theta_{2}\right)-\mu_{m}\left(\theta_{1}\right)\right|,
$$

we immediately obtain that B.115) is equivalent to

$$
d+\left|d \theta_{2}+\left(\theta_{2}-\theta_{1}\right) \mu_{m}\left(\theta_{1}\right)\right| \geq \delta .
$$

We now use the mean value Theorem on the map $\frac{1}{\tilde{B}\left(\theta^{2}\right)}$ and we thus obtain

$$
\frac{1}{\tilde{B}\left(\theta_{2}^{2}\right)}=\frac{1}{\tilde{B}\left(\theta_{1}^{2}\right)}+e(\xi)\left(\theta_{2}^{2}-\theta_{1}^{2}\right)
$$

where

$$
e(\xi)=-\frac{\tilde{B}^{\prime}\left(\xi^{2}\right)}{\tilde{B}\left(\xi^{2}\right)^{2}}>0,
$$

with $\xi \in\left(\theta_{1}, \theta_{2}\right)$. Actually, we can show that there exist two positive constants $e_{1}$ and $e_{2}$ such that

$$
0<e_{1} \leq e(\xi) \leq e_{2}, \quad \forall \xi \in\left[\alpha_{1}, \alpha_{2}\right] .
$$

We then easily obtain that

$$
d=\left|\frac{2 \pi}{\tilde{B}\left(\theta_{1}^{2}\right)}+2(m+1) \pi e(\xi)\left(\theta_{1}+\theta_{2}\right)\left(\theta_{1}-\theta_{2}\right)\right| .
$$

Using the triangle inequality we thus obtain that

$$
2(m+1) \pi e(\xi)\left(\theta_{1}+\theta_{2}\right)\left|\theta_{1}-\theta_{2}\right| \geq \frac{2 \pi}{\tilde{B}\left(\theta_{1}^{2}\right)}-d .
$$

We thus have to study different cases.

Case 1: If

$$
d \geq \frac{2 \pi}{\tilde{B}\left(\theta_{1}^{2}\right)}
$$

we easily obtain

$$
\left|\mu_{m+1}\left(\theta_{2}\right)-\mu_{m}\left(\theta_{1}\right)\right|+\left|\theta_{2} \mu_{m+1}\left(\theta_{2}\right)-\theta_{1} \mu_{m}\left(\theta_{1}\right)\right| \geq d \geq \frac{2 \pi}{\tilde{B}\left(\theta_{1}^{2}\right)} .
$$

Case 2: If

$$
d<\frac{2 \pi}{\tilde{B}\left(\theta_{1}^{2}\right)},
$$

then (B.117) gives us

$$
\left|\theta_{1}-\theta_{2}\right|>\frac{2 \pi-d \tilde{B}\left(\theta_{1}^{2}\right)}{2(m+1) \pi e(\xi)\left(\theta_{1}+\theta_{2}\right) \tilde{B}\left(\theta_{1}^{2}\right)} .
$$


Thus,

$$
\begin{aligned}
\mu_{m}\left(\theta_{1}\right)\left|\theta_{1}-\theta_{2}\right| & =\frac{2 m \pi}{\tilde{B}\left(\theta_{1}^{2}\right)}\left|\theta_{1}-\theta_{2}\right| \\
& >\frac{m}{m+1} \frac{2 \pi-d \tilde{B}\left(\theta_{1}^{2}\right)}{e(\xi)\left(\theta_{1}+\theta_{2}\right) \tilde{B}\left(\theta_{1}^{2}\right)^{2}} \\
& >\frac{2 \pi-d \tilde{B}\left(\alpha_{1}^{2}\right)}{4 e_{2} \alpha_{2} \tilde{B}\left(\alpha_{1}^{2}\right)^{2}}
\end{aligned}
$$

We note that

$$
d \theta_{2}<\frac{2 \pi-d \tilde{B}\left(\alpha_{1}^{2}\right)}{4 e_{2} \alpha_{2} \tilde{B}\left(\alpha_{1}^{2}\right)^{2}} \quad \Leftrightarrow \quad d<\frac{2 \pi}{\left(4 \theta_{2} e_{2} \alpha_{2} \tilde{B}\left(\alpha_{1}^{2}\right)+1\right) \tilde{B}\left(\alpha_{1}^{2}\right)} .
$$

If

$$
d>\frac{2 \pi}{\left(4 \theta_{2} e_{2} \alpha_{2} \tilde{B}\left(\alpha_{1}^{2}\right)+1\right) \tilde{B}\left(\alpha_{1}^{2}\right)},
$$

then as in the Case 1, we easily obtain

$$
\left|\mu_{m+1}\left(\theta_{2}\right)-\mu_{m}\left(\theta_{1}\right)\right|+\left|\theta_{2} \mu_{m+1}\left(\theta_{2}\right)-\theta_{1} \mu_{m}\left(\theta_{1}\right)\right| \geq d \geq \delta .
$$

If

$$
d<\frac{2 \pi}{\left(4 \theta_{2} e_{2} \alpha_{2} \tilde{B}\left(\alpha_{1}^{2}\right)+1\right) \tilde{B}\left(\alpha_{1}^{2}\right)}
$$

we then obtain

$$
\begin{aligned}
& \left|\mu_{m+1}\left(\theta_{2}\right)-\mu_{m}\left(\theta_{1}\right)\right|+\left|\theta_{2} \mu_{m+1}\left(\theta_{2}\right)-\theta_{1} \mu_{m}\left(\theta_{1}\right)\right| \\
= & d+\left|d \theta_{2}+\left(\theta_{2}-\theta_{1}\right) \mu_{m}\left(\theta_{1}\right)\right| \\
= & d+\left|\theta_{2}-\theta_{1}\right| \mu_{m}\left(\theta_{1}\right)-d \theta_{2} \\
> & d+\frac{2 \pi-d \tilde{B}\left(\alpha_{1}^{2}\right)}{4 e_{2} \alpha_{2} \tilde{B}\left(\alpha_{1}^{2}\right)^{2}}-d \theta_{2} \\
= & \frac{\pi}{2 e_{2} \alpha_{2} \tilde{B}\left(\alpha_{1}^{2}\right)^{2}}+d\left(1-\frac{1}{4 e_{2} \alpha_{2} \tilde{B}\left(\alpha_{1}^{2}\right)}-\theta_{2}\right) .
\end{aligned}
$$

We note that there exists $d_{0}>0$ such that for all $d<d_{0}$,

$$
d\left(1-\frac{1}{4 e_{2} \alpha_{2} \tilde{B}\left(\alpha_{1}^{2}\right)}-\theta_{2}\right)>-\frac{\pi}{4 e_{2} \alpha_{2} \tilde{B}\left(\alpha_{1}^{2}\right)^{2}} .
$$

Thus, for all $d<d_{0}$, we immediately obtain

$$
\left|\mu_{m+1}\left(\theta_{2}\right)-\mu_{m}\left(\theta_{1}\right)\right|+\left|\theta_{2} \mu_{m+1}\left(\theta_{2}\right)-\theta_{1} \mu_{m}\left(\theta_{1}\right)\right| \geq \frac{\pi}{4 e_{2} \alpha_{2} \tilde{B}\left(\alpha_{1}^{2}\right)^{2}} \geq \delta .
$$

Moreover, if $d \geq d_{0}$ we conclude as in the Case 1 .

We thus have shown that the curves of the first Figure are uniformly separated. Since, the same analysis is also true for the second Figure we have shown Lemma 4.6 . 


\section{Proof of Lemma 4.8}

The aim of this Appendix is to prove Lemma 4.8 which we recall here.

Lemma C.1. We set

$$
n(r)=\# E_{M} \cap B(0, r) \cap \mathcal{C},
$$

where

$$
E_{M}=\left\{\left(\left|\mu_{m}\right|,\left|\nu_{m}\right|\right), m \geq 1\right\}
$$

without multiplicity. Then,

$$
\varlimsup \frac{n(r)}{r^{2}}>0, \quad r \rightarrow+\infty .
$$

Proof. To prove the Lemma we use the work of Colin de Verdière on the coupled spectrum of commuting pseudodifferential operators in [18, 19. We recall that the operators $L$ and $H$ are defined by $(2.29)$ and satisfy (2.34). Since we proved in Lemma 2.5 that $H$ and $L$ are semibounded operators, we can say that there exists $M \in \mathbb{R}$ such that $L+M$ and $H+M$ are positive operators. We set

$$
P_{1}=\sqrt{L+M} \text { and } P_{2}=\sqrt{H+M} .
$$

The operators $P_{1}$ and $P_{2}$ are commuting, selfadjoint pseudodifferential operators of order 1 such that $P_{1}^{2}+P_{2}^{2}$ is an elliptic operator. These operators are thus in the framework of [18]. The principal symbol of $P_{1}$ and $P_{2}$ are given by

$$
p_{1}(x, \xi)=\sqrt{-\frac{s_{33}}{s^{11}} \xi_{2}^{2}+\frac{s_{23}}{s^{11}} \xi_{3}^{2}} \quad \text { and } \quad p_{2}(x, \xi)=\sqrt{\frac{s_{32}}{s^{11}} \xi_{2}^{2}-\frac{s_{22}}{s^{11}} \xi_{3}^{2}},
$$

respectively. We put

$$
p(x, \xi)=\left(p_{1}(x, \xi), p_{2}(x, \xi)\right),
$$

where $x:=\left(x^{2}, x^{3}\right), \xi:=\left(\xi_{2}, \xi_{3}\right)$ and $(x, \xi)$ is a point on the cotangent bundle of $\mathcal{T}^{2}$, i.e. $T^{\star} \mathcal{T}^{2}$. We will apply Theorem 0.7 of [18] to $P_{1}$ and $P_{2}$. We recall here this result adapted to our framework.

Theorem C.2. Let $C$ be a cone of $\dot{\mathbb{R}}^{2}=\mathbb{R}^{2} \backslash\{(0,0)\}$, with piecewise $C^{1}$ boundary such that $\partial C \cap W=\emptyset$, where $\partial C$ is the boundary of $C$ and $W$ is the set of critical values of $p$. We then have

$$
\#\{\lambda \in C \cap \Lambda,|\lambda| \leq r\}=\frac{1}{4 \pi^{2}} \operatorname{vol}_{\Omega}\left(p^{-1}(C \cap B(0, r))\right)+O(r),
$$

where $\Lambda$ is the coupled spectrum of $P_{1}$ and $P_{2}$ and $\Omega=d x^{2} \wedge d x^{3} \wedge d \xi_{2} \wedge d \xi_{3}$.

Thus, to use Theorem C.2. we have to determine the set $W$ of critical values of $p$. We first have to determine the critical points of $p$ i.e. the points for which the differential of $p$ is not onto. The differential of $p$ is given by (we omit the variables)

$$
D p(x, \xi)=\frac{-1}{4 p_{1} p_{2}}\left(\begin{array}{cccc}
\partial_{2}\left(\frac{s_{33}}{s^{11}}\right) \xi_{2}^{2}-\partial_{2}\left(\frac{s_{23}}{s^{11}}\right) \xi_{3}^{2} & \partial_{3}\left(\frac{s_{33}}{s^{11}}\right) \xi_{2}^{2}-\partial_{3}\left(\frac{s_{23}}{s^{11}}\right) \xi_{3}^{2} & 2 \frac{s_{33}}{s^{11}} \xi_{2} & -2 \frac{s_{23}}{s^{11}} \xi_{3} \\
-\partial_{2}\left(\frac{s_{32}}{s^{11}}\right) \xi_{2}^{2}+\partial_{2}\left(\frac{s_{22}}{s^{11}}\right) \xi_{3}^{2} & -\partial_{3}\left(\frac{s_{32}}{s^{11}}\right) \xi_{2}^{2}+\partial_{3}\left(\frac{s_{22}}{s^{11}}\right) \xi_{3}^{2} & -2 \frac{s_{32}}{s^{11}} \xi_{2} & 2 \frac{s_{22}}{s^{11}} \xi_{3}
\end{array}\right) .
$$

We compute the six $2 \times 2$ minors of this matrix and we search the points $(x, \xi)$ for which all these minors vanish. After calculation, we obtain that $(x, \xi)$ is a critical point of $p$ if and only if the four following conditions are satisfied:

$$
\left\{\begin{array}{l}
\xi_{2} \xi_{3}=0 \\
\xi_{3} \partial_{2}\left(s_{22}\right)\left(\xi_{2}^{2}+\xi_{3}^{2}\right)=0 \\
\xi_{2} \partial_{3}\left(s_{33}\right)\left(\xi_{2}^{2}+\xi_{3}^{2}\right)=0 \\
\partial_{2}\left(s_{22}\right) \partial_{3}\left(s_{33}\right)\left(\xi_{2}^{2}+\xi_{3}^{2}\right)^{2}=0
\end{array} .\right.
$$


Thus, there are four cases to study according to the vanishing of $\partial_{2}\left(s_{22}\right)$ and $\partial_{3}\left(s_{33}\right)$. We finally obtain that

$$
W=\left\{\begin{array}{l}
(0,0) \text { if } \partial_{2}\left(s_{22}\right) \neq 0 \quad \text { and } \quad \partial_{3}\left(s_{33}\right) \neq 0 \\
\mathcal{D}_{1} \text { if } \partial_{2}\left(s_{22}\right)=0 \text { and } \quad \partial_{3}\left(s_{33}\right) \neq 0 \\
\mathcal{D}_{2} \text { if } \partial_{2}\left(s_{22}\right) \neq 0 \text { and } \partial_{3}\left(s_{33}\right)=0 \\
\mathcal{D}_{1} \cup \mathcal{D}_{2} \text { if } \quad \partial_{2}\left(s_{22}\right)=0 \text { and } \quad \partial_{3}\left(s_{33}\right)=0
\end{array}\right.
$$

where

$$
\mathcal{D}_{1}=\left\{t\left(\sqrt{s_{23}}, \sqrt{-s_{22}}\right), t \geq 0\right\} \quad \text { and } \quad \mathcal{D}_{2}=\left\{t\left(\sqrt{-s_{33}}, \sqrt{s_{32}}\right), t \geq 0\right\},
$$

where $s_{22}, s_{23}=s_{22}+1, s_{33}$ and $s_{32}=s_{33}+1$ are constants according to the case we study. We now recall that in Theorem C.2. we have to choose a cone $C$ such that $\partial C \cap W=\emptyset$ and we want to study the set

$$
p^{-1}(C \cap B(0, r))=p^{-1}(C) \cap p^{-1}(B(0, r)) .
$$

Let $r>0$, we first study the set $p^{-1}(B(0, r))$. We recall that there exists a constant $c_{1}>0$ such that

$$
\max \left(-\frac{s_{33}}{s^{11}}, \frac{s_{23}}{s^{11}}, \frac{s_{32}}{s^{11}},-\frac{s_{22}}{s^{11}}\right) \leq c_{1} .
$$

Thus, if $\left(\xi_{2}, \xi_{3}\right) \in B\left(0, \frac{r}{\sqrt{2 c_{1}}}\right)$ and $\left(x^{2}, x^{3}\right) \in \mathcal{T}^{2}$, then

$$
\|p(x, \xi)\|=\sqrt{p_{1}(x, \xi)+p_{2}(x, \xi)} \leq \sqrt{2 c_{1}\left(\xi_{2}^{2}+\xi_{3}^{2}\right)} \leq r .
$$

We deduce from this fact that

$$
\mathcal{T}^{2} \times B\left(0, \frac{r}{\sqrt{2 c_{1}}}\right) \subset p^{-1}(B(0, r)) .
$$

We now study the set $p^{-1}(C)$. We have to divide our study in four cases as we have seen before.

Case 1: $\partial_{2}\left(s_{22}\right) \neq 0$ and $\partial_{3}\left(s_{33}\right) \neq 0$. In this case we just have to avoid the point $\{(0,0)\}$. We consider the cone

$$
C=\left\{(x, y) \in \mathbb{R}^{2} \text { such that } \epsilon \leq x, \epsilon \leq y\right\}, \quad \epsilon>0 .
$$

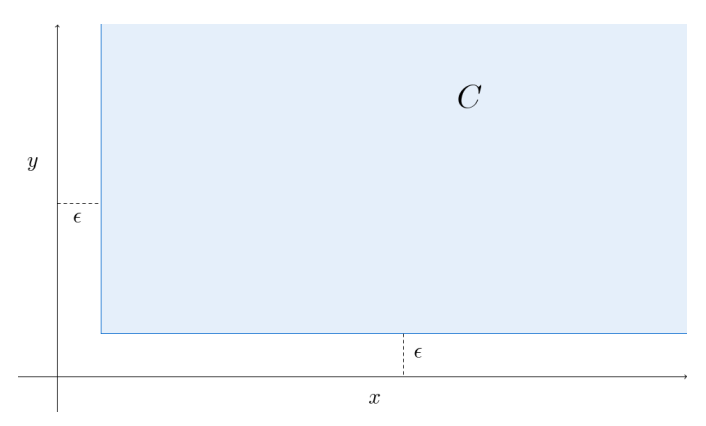

Figure 5: Case 1 
By definition

$$
p^{-1}(C)=\left\{(x, \xi) \in \mathcal{T}^{2} \times \mathbb{R}^{2}, \epsilon \leq p_{1}(x, \xi), \epsilon \leq p_{2}(x, \xi)\right\}
$$

and since there exists $c_{2}>0$ such that

$$
c_{2} \leq \min \left(-\frac{s_{33}}{s^{11}}, \frac{s_{23}}{s^{11}}, \frac{s_{32}}{s^{11}},-\frac{s_{22}}{s^{11}}\right),
$$

there exists $\eta>0$ such that

$$
\mathcal{T}^{2} \times\left(\mathbb{R}^{2} \backslash B(0, \eta)\right) \subset p^{-1}(C) .
$$

Case 2: $\partial_{2}\left(s_{22}\right)=0$ and $\partial_{3}\left(s_{33}\right) \neq 0$. We have to avoid the half-line $\mathcal{D}_{1}$ which has slope $\beta_{1}=\sqrt{\frac{-s_{22}}{s_{23}}}$. We consider the cone

$$
C=\left\{(x, y) \in \mathbb{R}^{2} \text { such that } \epsilon \leq x, \epsilon \leq y \leq \beta_{1} x-\epsilon\right\}, \quad \epsilon>0 .
$$

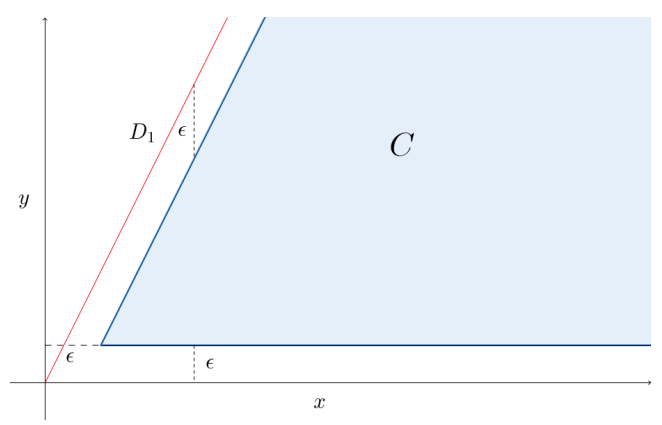

Figure 6: Case 2

As in the first case, there is $\eta>0$ such that

$$
p_{1}(x, \xi) \geq \epsilon, \quad \forall(x, \xi) \in \mathcal{T}^{2} \times\left(\mathbb{R}^{2} \backslash B(0, \eta)\right)
$$

and

$$
p_{2}(x, \xi) \geq \epsilon, \quad \forall(x, \xi) \in \mathcal{T}^{2} \times\left(\mathbb{R}^{2} \backslash B(0, \eta)\right) .
$$

The last condition can be rewritten as

$$
\begin{aligned}
p_{2}(x, \xi) \leq \beta_{1} p_{1}(x, \xi)-\epsilon & \Leftrightarrow \sqrt{\frac{s_{32}}{s^{11}} \xi_{2}^{2}-\frac{s_{22}}{s^{11}} \xi_{3}^{2}} \leq \sqrt{\frac{-s_{22}}{s_{23}}} \sqrt{-\frac{s_{33}}{s^{11}} \xi_{2}^{2}+\frac{s_{23}}{s^{11}} \xi_{3}^{2}}-\epsilon \\
& \Leftrightarrow \sqrt{\frac{s_{32}}{s^{11}} \xi_{2}^{2}-\frac{s_{22}}{s^{11}} \xi_{3}^{2}} \leq \sqrt{\frac{s_{22} s_{33}}{s_{23} s^{11}} \xi_{2}^{2}-\frac{s_{22}}{s^{11}} \xi_{3}^{2}}-\epsilon .
\end{aligned}
$$

We recall that, thanks to the condition given in Remark 1.18 ,

$$
\frac{s_{22} s_{33}}{s_{23}}>s_{32} \text {. }
$$

Thus, there exists $\epsilon>0$ small enough such that

$$
p_{2}(x, \xi) \leq \beta_{1} p_{1}(x, \xi)-\epsilon, \quad \forall(x, \xi) \in \mathcal{T}^{2} \times \mathbb{R}^{2} .
$$


Finally, we have shown that for such an $\epsilon$, there exists $\eta>0$ such that

$$
\mathcal{T}^{2} \times\left(\mathbb{R}^{2} \backslash B(0, \eta)\right) \subset p^{-1}(C) .
$$

Case 3: $\partial_{2}\left(s_{22}\right) \neq 0$ and $\partial_{3}\left(s_{33}\right)=0$. We have to avoid the half-line $\mathcal{D}_{2}$ which has slope $\beta_{2}=\sqrt{-\frac{s_{32}}{s_{33}}}$. We consider the cone

$$
C=\left\{(x, y) \in \mathbb{R}^{2} \text { such that } \epsilon \leq x, \beta_{2} x+\epsilon \leq y\right\}, \quad \epsilon>0,
$$

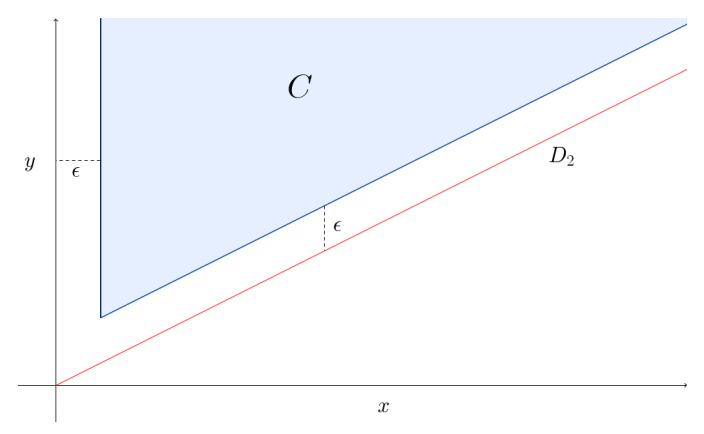

Figure 7: Case 3

and we show, as in the second case, that for $\epsilon>0$ small enough there exists $\eta>0$ such that

$$
\mathcal{T}^{2} \times\left(\mathbb{R}^{2} \backslash B(0, \eta)\right) \subset p^{-1}(C) .
$$

Case 4: $\partial_{2}\left(s_{22}\right)=0$ and $\partial_{3}\left(s_{33}\right)=0$. We have to avoid $\mathcal{D}_{1} \cup \mathcal{D}_{2}$ which have slopes $\alpha_{1}$ and $\alpha_{2}$ respectively. We consider the cone

$$
C=\left\{(x, y) \in \mathbb{R}^{2} \text { such that } \epsilon \leq x, \beta_{2} x+\epsilon \leq y \leq \beta_{1} x-\epsilon\right\}, \quad \epsilon>0 .
$$

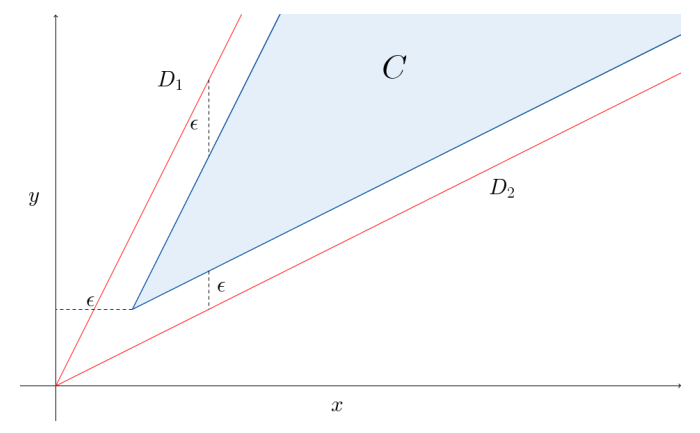

Figure 8: Case 4

As in the first case, there is $\eta>0$ such that

$$
p_{1}(x, \xi) \geq \epsilon, \quad \forall(x, \xi) \in \mathcal{T}^{2} \times\left(\mathbb{R}^{2} \backslash B(0, \eta)\right)
$$


and, as in the second and the third cases, there exists $\epsilon>0$ small enough such that

$$
p_{2}(x, \xi) \leq \beta_{1} p_{1}(x, \xi)-\epsilon, \quad \forall(x, \xi) \in \mathcal{T}^{2} \times \mathbb{R}^{2}
$$

and

$$
\beta_{2} p_{1}(x, \xi)+\epsilon \leq p_{2}(x, \xi), \quad \forall(x, \xi) \in \mathcal{T}^{2} \times \mathbb{R}^{2} .
$$

Thus, for $\epsilon>0$ small enough, there exists $\eta>0$ such that

$$
\mathcal{T}^{2} \times\left(\mathbb{R}^{2} \backslash B(0, \eta)\right) \subset p^{-1}(C) .
$$

In conclusion, we have shown that in any cases there exists $\eta>0$ such that

$$
\mathcal{T}^{2} \times\left(\mathbb{R}^{2} \backslash B(0, \eta)\right) \subset p^{-1}(C) .
$$

Moreover, in each case the cone $\mathcal{C}$ defined in 4.86 is, by definition, included in the cone $C$ we considered and we can thus apply Theorem C.2 to this cone. Therefore, thanks to $\overline{C .119}-(\bar{C} .120)$ we thus have shown that for $r>0$ large enough

$$
\mathcal{T}^{2} \times\left(B\left(0, \frac{r}{\sqrt{2 c_{1}}}\right) \backslash B(0, \eta)\right) \subset p^{-1}(\mathcal{C}) \cap p^{-1}(B(0, r))=p^{-1}(\mathcal{C} \cap B(0, r)) .
$$

From the inclusion C.121 we can deduce that there exists a constant $c>0$ such that

$$
c r^{2} \leq \frac{1}{4 \pi^{2}} \operatorname{vol}_{\Omega}\left(B\left(0, \frac{r}{\sqrt{2 c_{1}}}\right) \backslash B(0, \eta)\right) \leq \frac{1}{4 \pi^{2}} \operatorname{vol}_{\Omega}\left(p^{-1}(\mathcal{C} \cap B(0, r))\right) .
$$

Thanks to Theorem C.2. we can then conclude that there exists $c>0$ such that

$$
\#\{\lambda \in \mathcal{C} \cap \Lambda,|\lambda| \leq r\} \geq c r^{2} .
$$

Finally, we recall that

$$
\Lambda=\left\{\left(\sqrt{\mu_{m}^{2}+M}, \sqrt{\nu_{m}^{2}+M}\right), \quad m \geq 1\right\}
$$

and we note that, thanks to the fact that $\mu_{m}^{2} \rightarrow+\infty$ and $\nu_{m}^{2} \rightarrow+\infty$, as $m \rightarrow+\infty$,

$$
\sqrt{\mu_{m}^{2}+M} \sim\left|\mu_{m}\right| \text { and } \sqrt{\nu_{m}^{2}+M} \sim\left|\nu_{m}\right|, \quad m \rightarrow+\infty .
$$

We recall that

$$
n(r)=\#\left\{\lambda \in \mathcal{C} \cap E_{M},|\lambda| \leq r\right\},
$$

without multiplicity, whereas the result obtained before was computed counting multiplicity. However, the multiplicity of the coupled eigenvalues is at most 4 (see Remark 2.7). Thus, even if it means divide by 4 , we can conclude that

$$
\varlimsup \frac{n(r)}{r^{2}}>0, \quad r \rightarrow+\infty .
$$

Acknowledgments: This paper was initiated by Thierry Daudé and François Nicoleau while the author was visiting Thierry Daudé and Niky Kamran at McGill University during his PhD. The author wants to deeply thank Thierry Daudé and François Nicoleau for their help and their support and Niky Kamran for his hospitality and encouragement. The author also would like to warmly thank Georgi Popov and Thomas Wallez for useful discussions on the study of the coupled spectrum. Finally, the author also would like to thank his friends and colleagues Valentin Samoyeau and Pierre Vidotto for their support. 


\section{References}

[1] de Alfaro V., Regge T., Potential scattering, North-Holland Publishing Co., Amsterdam; Interscience Publishers Division John Wiley ans Sons, Inc., (1965).

[2] Arnold V.I., Mathematical methods of classical mechanics, Springer Science \& Business Media, Vol. 60, (2013).

[3] Benenti S., Separability in Riemannian manifolds, Preprint (2015), ArXiv:1512.07833.

[4] Benenti S., Orthogonal separable dynamical systems, Math. Publ., Silesian Univ. Opava, 1, (1993), 163-184.

[5] Benenti S., Chanu C., Rastelli G., Remarks on the connection between the additive separation of the Hamilton-Jacobi equation and the multiplicative separation of the Schrödinger equation. I. The completeness and Robertson condition, Journal of Mathematical Physics 43, 5183, (2002).

[6] Benenti S., Chanu C., Rastelli G., Remarks on the connection between the additive separation of the Hamilton-Jacobi equation and the multiplicative separation of the Schrödinger equation. II. First integrals and symmetry operators, Journal of Mathematical Physics 43, 5223, (2002).

[7] Benenti S., Francaviglia M., The theory of separability of the Hamilton-Jacobi equation and its applications to general relativity, in General relativity and gravitation, Vol. 1, Plenum, New York-London, (1980), 393-439.

[8] Bennewitz C., A proof of the local Borg-Marchenko Theorem, Comm. Math. Phys. 211, (2001), 131-132.

[9] Berndtsson B., Zeros of analytic functions of several variables, Ark. Mat. 16 (1978), no. 2, 251-262.

[10] Bloom T., A spanning set for $\mathcal{C}\left(I^{n}\right)$, Trans. Amer. Math. Soc. 321, (1990), no.2, 741-759.

[11] Boas R.P., Entire functions, Academic Press Inc., New York (1954).

[12] Bolsinov A. V., Matveev. V. S., Local normal forms for geodesically equivalent pseudoRiemannian metrics, Trans. Amer. Math. Soc. 9, (2015), 6719-6749.

[13] Bonthwick D., Spectral theory for infinite-area hyperbolic surface, Birkhäuser, Boston-Basel-Berlin, (2007).

[14] Borthwick D., Perry P. A., Inverse scattering results for manifolds hyperbolic near infinity, J. of Geom. Anal. 21, No. 2, (2011), 305-333.

[15] Bouclet J.M., Absence of eigenvalue at the bottom of the continuous spectrum on asymptotically hyperbolic manifolds, Ann. Global Anal. Geom. 44, no 2, (2013), 115-136.

[16] Chalendar I., Partington J., Multivariable approximate Carleman-type theorems for complex measures, Ann. Probab. 35 (2007), no.1, 384-396.

[17] Chanu C., Rastelli G., Fixed energy R-separation for Schrödinger equation, Int. J. Geom. Methods Mod. Phys. 3 (2006), no. 3, 489-508.

[18] Colin de Verdière Y., Spectre conjoint d'opérateurs pseudo-différentiels qui commutent. I. Le cas non intégrable, Duke Math. J. 46 (1979), no. 1, 169-182. 
[19] Colin de Verdière Y., Spectre conjoint d'opérateurs pseudo-différentiels qui commutent. II. Le cas intégrable, Math. Z. 171 (1980), no. 1, 51-73.

[20] Coluins P.D.B., An introduction to Regge theory and high energy physics, Cambridge Monographs on Mathematical Physics, Vol. 4, (1977).

[21] Daudé T., Gobin D., Nicoleau F., Local inverse scattering results at fixed energy in spherically symmetric asymptotically hyperbolic manifolds, To appear in Inverse Problems and Imaging, Preprint (2013), ArXiv:1310.0733.

[22] Daudé T., Kamran N., Nicoleau F., Inverse scattering at fixed energy on asymptotically hyperbolic Liouville surfaces, To appear in Inverse problems, Preprint (2015), ArXiv:1409.6229.

[23] Daudé T., Kamran N., Nicoleau F., Non-uniqueness results for the anisotropic Calderon problem with data measured on disjoint sets, Preprint (2015), ArXiv:1510.06559.

[24] Daudé T., Nicoleau F. Direct and inverse scattering at fixed energy for massless charged Dirac fields by Kerr-Newman-De Sitter black holes, Preprint (2013), ArXiv:1307.2842, to be published in Memoirs of the AMS.

[25] Daudé T., Nicoleau F. Inverse scattering at fixed energy in de Sitter-Reissner-Nordström black holes, Ann. Henri Poincaré 12, (2011), 1-47.

[26] Daudé T., Nicoleau F. Local inverse scattering at a fixed energy for radial Schrödinger operators and localization of the Regge poles, Preprint (2015), ArXiv:1502.02276, to be published in Annales Henri Poincaré.

[27] Dos Santos Ferreira D., Kenig C. E., Salo M., Uhlmann G. Limiting Carleman weights and anisotropic inverse problems, Invent. Math., 178 no. 1, (2009), 119-171.

[28] Dos Santos Ferreira D., Kurylev Y., Lassas M., Salo M. The Calderón problem in transversally anisotropic geometries, Preprint (2013), ArXiv:1305.1273, to be published in Journal of European Mathematical Society.

[29] Eisenhart L.P., Separable systems of Staeckel, Annals of Math., 35 (1934), no. 2, 284D305.

[30] Eisenhart L.P., Riemannian geometry, Princeton University Press, Princeton, NJ (1997).

[31] Freiling G., Yurko V., Inverse Sturm-Liouville problems and their applications, NOVA Science Publishers New York, (2001).

[32] Freiling G., Yurko V., Inverse problems for differential operators with singular boundary conditions, Math. Nachr. 278 no. 12-13, (2005), 1561-1578.

[33] Gesztesy F., Simon B., On local Borg-Marchenko uniqueness results, Comm. Math. Phys. 211, (2000), 273-287.

[34] Gobin D., Inverse scattering at fixed energy for massive charged Dirac fields in de Sitter-ReissnerNordström black holes, Inverse Problems 31, (2015).

[35] Guillarmou C., Sá Barreto A., Inverse problems for Einstein manifolds, Inverse Probl. Imaging 3, (2009), 1-15. 
[36] Guillarmou C., Sá Barreto A., Scattering and inverse scattering on ACH manifolds, J. Reine Angew. Math. 622, (2008), 1-55.

[37] Gulllarmou C., Tzou L., Calderón inverse problem with partial data on Riemann surfaces, Duke Math. J., 158, (2011), 83-120.

[38] Guillarmou C., Tzou L., The Calderon inverse problem in two dimensions. Inverse problems and applications: Inside Out. II, 119-166, Math. Sci. Res. Inst. Publ. 60, Cambridge University Press, Cambridge, (2013).

[39] Hormander L., An introduction to complex analysis in several variables, Elsevier, 7, (1973).

[40] Isozaki H., Kurylev J., Introduction to spectral theory and inverse problems on asymptotically hyperbolic manifolds, MSJ Memoirs 32, Preprint (2014), ArXiv:1102.5382.

[41] Isozaki H., Kurylev Y., Lassas M., Conic singularities, generalized scattering matrix, and inverse scattering on asymptotically hyperbolic surfaces, Journal fur die reine und angewandte Mathematik (Crelle's journal), (2011).

[42] Joshi M.S., Sá Barreto A., Inverse scattering on asymptotically hyperbolic manifolds, Acta Mathematica, 184, (2000), 41-86.

[43] Kalnins E.G., Miller JR. W., Intrinsic characterisation of orthogonal $R$ separation for Laplace equations, J. Phys. A 15 (1982), no. 9, 2699-2709.

[44] Kalnins E.G., Miller JR. W., Killing tensors and variable separation for Hamilton-Jacobi and Helmholtz equations, SIAM J. Math. Anal. 11, (1980), 1011-1026.

[45] Kalnins E.G., Miller JR. W., The theory of orthogonal R-separation for Helmholtz equations, Adv. in Math. 51 (1984), no. 1, 91-106.

[46] Katchalov A., Kurylev Y., Lassas M., Inverse boundary spectral problems, Chapman and Hall/CRC (2001) 290 pp.

[47] Kenig C., Salo M., The Calderón problem with partial data on manifolds and applications, Anal. PDE, 6 no. 8, (2013), 2003-2048.

[48] Kenig C., Salo M., Recent progress in the Calderon problem with partial data, Contemp. Math. 615, (2014), 193-222.

[49] Koornwinder T.H., A precise definition of separation of variables, Proc. Fourth Scheveningen Conf., Scheveningen, (1979), Lecture Notes in Math., Springer, Berlin (1980).

[50] Kostenko A., Sakhnovich A., Teschl G., Weyl-Titchmarsh theory for Schrödinger operators with strongly singular potentials, Int. Math. Res. Not. 2012, (2012), 1699-1747.

[51] Lassas M., Taylor M., Uhlmann G., The Dirichlet-to-Neumann map for complete Riemannian manifolds with boundary, Comm. Anal. Geom., 11, (2003), 207-221.

[52] Lassas M., Uhlmann G., On determining a Riemannian manifold from the Dirichlet-to-Neumann map, Ann. Sci. École Norm. Sup. (4), 34, (2001), 771-787.

[53] Lebedev N. N., Special functions and their applications, Prentice-Hall, Englewood Cliffs (1965). 
[54] Lee J. M., Uhlmann G., Determining anisotropic real-analytic conductivities by boundary measurements, Comm. Pure Appl. Math., 42 no. 8, (1989), 1097-1112.

[55] Levi-Civita T., Sulla integrazione della equazione di Hamilton-Jacobi per separazione di variabili, Math. Ann., 59, (1904), 383-397.

[56] Marazzi L., Inverse scattering on conformally compact manifolds, Inverse Probl. Imaging, 3, (2009), $537-550$.

[57] Mazzeo, R., Melrose R. B., Meromorphic extension of the resolvent on complete spaces with asymptotically constant negative curvature, J. Funct. Anal. 75, (1987), 260-310.

[58] Melrose R. B., Geometric scattering theory, Cambridge University Press, Cambridge, (1995).

[59] Miller JR. W., Mechanisms for variable separation in partial differential equations and their relationship to group theory, Symmetries and nonlinear phenomena 9, (1988), 188-221.

[60] Musin, I. KH., On the Fourier-Laplace representation of analytic functions in tube domains, Collect. Math. 45 (1994), no. 3, 301-308.

[61] Newton R.G., Scattering theory of waves and particles, Dover Publications, Mineola (2002) (Reprint of the 1982 second edition New York: Springer, with list of errata prepared for this edition by the author)

[62] Ramm A.G. An inverse scattering problem with part of the fixed-energy phase shifts, Comm. Math. Phys. 207(1), (1999), 231-247.

[63] RegGe T. Introduction to complex orbita momenta, Nuevo Cimento XIV(5), (1959), 951-976.

[64] Robertson H. P. Bemerkung über separierbare Systeme in der Wellenmechanik, Math. Ann., 98, (1928), 749-752.

[65] SÁ BARReto A., Radiation fields, scattering, and inverse scattering on asymptotically hyperbolic manifolds, Duke Math. J., 129, (2005), 407-480.

[66] Salo M., The Calderón problem on Riemannian manifolds, Inverse problems and applications: inside out. II, Math. Sci. Res. Inst. Publ., 60, Cambridge Univ. Press, Cambridge, (2013), 167-247.

[67] Šapovalov, V. N., Stäckel spaces, Sibirsk. Mat. Zh., 20, (1979), 1117-1130, 1168.

[68] StÄCKel P., Über die integration der Hamilton-Jacobischen differentialgleichung mittelst separation der variabel, Habilitationsschirft, Ph.D. thesis Halle, (1891).

[69] Stäckel P., Ueber die Bewegung eines Punktes in einer n-fachen Mannigfaltigkeit, Math. Ann., 42, (1893), 537-563.

[70] Teschl G., Mathematical Methods in Quantum Mechanics, Graduate Studies in Mathematics Vol. 99, AMS Providence, Rhode Island, (2009).

[71] Uhlmann G., Electrical impedance tomography and Calderon's problem, Inverse Problems 25, (2009), 123011, 39p. 